ALEA, Lat. Am. J. Probab. Math. Stat. 18, 1395-1439 (2021)

DOI: $10.30757 /$ ALEA.v18-51

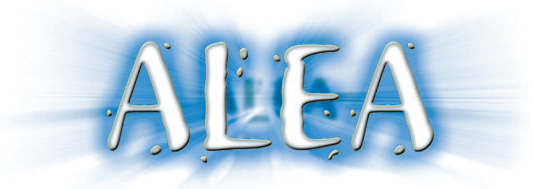

\title{
A new look at the interfaces in percolation
}

\section{Raphaël Cerf and Wei Zhou}

DMA, Ecole Normale Supérieure, CNRS, PSL Research University, 75005 Paris.

Laboratoire de Mathématiques d'Orsay,

Université Paris-Sud, CNRS, Université Paris-Saclay, 91405 Orsay.

E-mail address: raphael.cerf@ens.fr, wei.zhou@ens.fr

URL: https://ww. math.u-psud.fr/ cerf/, https://sites.google.com/view/wzhou/home

\begin{abstract}
We propose a new definition of the interface in the context of the Bernoulli percolation model. We construct a coupling between two percolation configurations, one which is a standard percolation configuration, and one which is a percolation configuration conditioned on a disconnection event. We define the interface as the random set of the edges where these two configurations differ. We prove that, inside a cubic box $\Lambda$, the interface between the top and the bottom of the box is typically localised within a distance of order $(\ln |\Lambda|)^{2}$ of the set of the pivotal edges.
\end{abstract}

\section{Introduction}

At the macroscopic level, the interface between two pure phases seems to be deterministic. In fact, such an interface obeys a minimal action principle: it minimizes the surface tension between the two phases and it is close to the solution of a variational problem. This can be seen as an empirical law, derived from the observation at the macroscopic level. This law has been justified from a microscopic point of view in the context of the Ising model by Dobrushin, Kotecky and Shlosman (see Dobrushin et al., 1992). One starts with a simple model of particles located on a discrete lattice. There are two types of particles, which have a slight tendency to repel each other. In the limit where the number of particles tends to $\infty$, at low temperatures, the system presents a phenomenon of phase segregation, with the formation of interfaces between two pure phases. On a suitable scale, these interfaces converge towards deterministic shapes, a prominent example being the Wulff crystal of the Ising model, which is the typical shape of the Ising droplets. Although the limit is deterministic on the macroscopic level, the interfaces are intrinsically random objects and their structure is extremely complex. In two dimensions, the fluctuations of the Ising interfaces were precisely analysed in the DKS theory, with the help of cluster expansions in Dobrushin and Hryniv (1997); Dobrushin et al. (1992). A non-perturbative analysis of Ising interfaces was subsequently developed, using completely different techniques, see Ioffe (1994, 1995); Ioffe and Schonmann (1998); Greenberg and Ioffe (2005); Campanino et al. (2003).

Received by the editors March 27th, 2020; accepted February 11th, 2021.

2010 Mathematics Subject Classification. 60K35, 82B20, 82B43.

Key words and phrases. Percolation, interface, coupling. 
In higher dimensions, there is essentially one result on the fluctuations of the interfaces, due to Dobrushin (1972), which says that horizontal interfaces stay localised at low temperatures. When dealing with interfaces in the Ising model, the first difficulty is to get a proper definition of the interface itself. The usual way is to start with the Dobrushin type boundary conditions, that is a box with pluses on its upper half boundary and minuses on its lower half boundary. This automatically creates an Ising configuration in the box with a microscopic interface between the pluses and the minuses which separates the upper half and the lower half of the box. Yet it is still not obvious how one should define the interface in this case, because several such microscopic interfaces exist, and a lot of different choices are possible. Dobrushin, Kotecky and Shlosman (see Dobrushin et al., 1992) introduced a splitting rule between contours, which leads to pick up one particular microscopic interface. The potential problem with this approach is that the outcome is likely to include microscopic interfaces which are not necessarily relevant, for instance interfaces between opposite signs which would have been present anyway, and which are not induced by the Dobrushin boundary conditions. Ultimately, we would like to have a definition of the interface which is not limited to low temperatures and which allows to derive results until the critical point.

Our goal here is to propose a new way to look at the random interfaces, in any dimension $d \geq 2$. We start our investigation in the framework of the Bernoulli percolation model, for several reasons. First, the probabilistic structure of the percolation model is simpler than the one of the Ising model. Another reason is that the Wulff Theorem in dimensions three was first derived for the percolation model (see Cerf, 2000) and then extended to the Ising model in Bodineau (1999); Cerf and Pisztora (2000). A key fact was that the definition of the surface tension is much simpler for the percolation model than for the Ising model. This leads naturally to hope that the probabilistic structure of the interfaces should be easier to apprehend as well in the percolation model. Finally, in the context of percolation, one sees directly which edges are essential or not in an interface: these are the pivotal edges. There is no corresponding notion in the Ising model. For all these reasons, it seems wise to try to develop a probabilistic description of random interfaces in the framework of Bernoulli percolation.

In this paper, we consider the Bernoulli bond percolation model with a parameter $p$ close to 1 . Interfaces in a cubic box $\Lambda$ are naturally created when the configuration is conditioned on the event that the top side $T$ and the bottom side $B$ of the box are disconnected. From now onwards, this event is denoted by

$$
\{T \longleftrightarrow B\}
$$

Our goal is to gain some understanding on the typical configurations realizing such a disconnection event. To do so, we build a coupling between two percolation configurations $X, Y$ in the box $\Lambda$ such that:

- The edges in $X$ are i.i.d., open with probability $p$ and closed with probability $1-p$.

- The distribution of $Y$ is the distribution of the Bernoulli percolation conditioned on $\{T \longleftrightarrow B\}$. - Every edge open in $Y$ is also open in $X$.

We define then the random interface between the top side $T$ and the bottom side $B$ of the box $\Lambda$ as the random set $\mathcal{I}$ of the edges where $X$ and $Y$ differ:

$$
\mathcal{I}=\{e \subset \Lambda: X(e) \text { is open, } Y(e) \text { is closed }\} .
$$

Among these edges, some are essential for the disconnection between $T$ and $B$ to occur. These edges are called pivotal and they are denoted by $\mathcal{P}$ :

$$
\mathcal{P}=\left\{e \in \mathcal{I}: \quad \begin{array}{c}
\text { the opening of } e \text { in } Y \text { would create } \\
\text { an open connection between } T \text { and } B
\end{array}\right\} .
$$

When conditioning on the disconnection between $T$ and $B$, a lot of pivotal edges are created. Yet another collection of edges which are not essential for the disconnection event turn out to be closed as well. Therefore it becomes extremely difficult to understand the effect of the conditioning on 
the distribution by looking at the conditioned probability measure alone. This is why we build a coupling and we define the interface as the set of the edges where the two percolation configurations differ. The set $\mathcal{P}$ of the pivotal edges can be detected by a direct inspection of the conditioned configuration, but not the interface $\mathcal{I}$. Our main result provides a quantitative control on the interface $\mathcal{I}$ with respect to the set $\mathcal{P}$ of the pivotal edges. We denote by $\mu_{p}$ the coupling probability measure between the configurations $X$ and $Y$. The precise construction of $\mu_{p}$ is done in Section 2 . We denote by $d$ the usual Euclidean distance on $\mathbb{R}^{d}$, by $\Lambda$ a cubic box with sides parallel to the axis of $\mathbb{Z}^{d}$, and by $|\Lambda|$ the cardinality of $\Lambda \cap \mathbb{Z}^{d}$.

Theorem 1.1. There exists $\tilde{p}<1$ and $\kappa>0$, such that, for $p \geqslant \tilde{p}$, any $c \geqslant 1$ and any box $\Lambda$ satisfying $|\Lambda| \geqslant \max \left\{(c d)^{c d^{2}}, 3^{6 d}\right\}$,

$$
\mu_{p}\left(\exists e \in \mathcal{P} \cup \mathcal{I}, d\left(e, \Lambda^{c} \cup \mathcal{P} \backslash\{e\}\right) \geqslant \kappa c^{2} \ln ^{2}|\Lambda|\right) \leqslant \frac{1}{|\Lambda|^{c}} .
$$

The typical picture which emerges from Theorem 1.1 is the following. In the configuration conditioned on the event $\{T \longleftrightarrow B\}$, there is a set $\mathcal{P}$ of pivotal edges. These are the edges having one extremity connected by an open path to the top $T$ and the other extremity connected by an open path to the bottom $B$. Because of the conditioning, compared to the i.i.d. configuration, some additional edges are closed, but they are typically within a distance of order $(\ln |\Lambda|)^{2}$ of the set $\mathcal{P}$ of the pivotal edges. The edges which are further away from $\mathcal{P}$ behave as in the ordinary unconditioned percolation. Therefore the interface $\mathcal{I}$ is strongly localised around the set $\mathcal{P}$ of the pivotal edges. The interface is a dust of closed edges pinned around the pivotal edges.

Our next endeavour was to obtain a conditional version of Theorem 1.1. More precisely, we would like to estimate the conditional probability

$$
\mu_{p}\left(e \in \mathcal{P} \cup \mathcal{I} \mid d(e, \mathcal{P} \backslash\{e\}) \geqslant \kappa(\ln |\Lambda|)^{2}\right) .
$$

We did not really succeed so far, however we are able to control the interface conditionally on the distance to a cut. Before stating our result, let us recall the definition of a cut.

Definition 1.2. A set $S$ of edges separates the top $T$ and the bottom $B$ in $\Lambda$ if every deterministic path of edges from $T$ to $B$ in $\Lambda$ intersects $S$. A cut $C$ between $T$ and $B$ in $\Lambda$ is a set of edges which separates $T$ and $B$ in $\Lambda$ and which is minimal for the inclusion.

A cut $C$ is closed in the configuration $Y$ if all the edges of $C$ are closed in $Y$. We denote by $\mathcal{C}$ the collection of the closed cuts present in $Y$. Since $Y$ realizes the event $\{T \leftrightarrow \leftrightarrow B\}$, the collection $\mathcal{C}$ is not empty.

Theorem 1.3. We have the following inequality:

$$
\begin{array}{rlrr}
\exists \tilde{p}<1 \quad \exists \kappa>0 \quad \forall p \geqslant \tilde{p} \quad \forall c \geqslant 2 \quad \forall \Lambda \quad & \ln |\Lambda|>4+c+2 d c^{2}+12(2 \kappa d)^{d} \\
\forall e \in \Lambda & d\left(e, \Lambda^{c}\right) \geqslant \kappa c^{2} \ln ^{2}|\Lambda| \\
& \mu_{p}\left(e \in \mathcal{I}\left|\exists C \in \mathcal{C}, d(e, C) \geqslant \kappa c^{2} \ln ^{2}\right| \Lambda \mid\right) \leqslant \frac{1}{|\Lambda|^{c}} .
\end{array}
$$

Let us explain briefly how we build the coupling probability measure $\mu_{p}$, as well as the strategy for proving Theorem 1.1. Conditioning on the event $\{T \leftrightarrow B\}$ creates non trivial correlations between the edges, and there is no simple tractable formula giving for instance the conditional distribution of a finite set of edges. Yet a standard application of the FKG inequality yields that, for any increasing event $A$, we have

$$
P_{p}(A \mid T \longleftrightarrow B) \leq P_{p}(A)
$$


Thus the product measure $P_{p}$ stochastically dominates the conditional measure $P_{p}(\cdot \mid T \longleftrightarrow B)$. Strassen's Theorem tells us that there exists a monotone coupling between these two probability measures. In order to derive quantitative estimates on the differences between the coupled configurations, we build our coupling measure as the invariant measure of a dynamical process. This method of coupling is standard, for instance it is used in the proof of Holley inequality (see Chapter 2 of Grimmett, 1995). Our contribution is to study some specific properties of this coupling in the context of percolation, and to relate it to the geometry of the interfaces. To do so, we consider the classical dynamical percolation process in the box $\Lambda$, see Steif (2009). Since we always work in a finite box, we use the following discrete time version. We start with an initial configuration $X_{0}$. At each step, we choose one edge uniformly at random, and we update its state with a coin of parameter $p$. Of course all the random choices are independent. The resulting process is denoted by $\left(X_{t}\right)_{t \in \mathbb{N}}$. Obviously the invariant probability measure of $\left(X_{t}\right)_{t \in \mathbb{N}}$ is the product measure $P_{p}$ and the process $\left(X_{t}\right)_{t \in \mathbb{N}}$ is reversible with respect to $P_{p}$. Next, we duplicate the initial configuration $X_{0}$, thereby getting a second configuration $Y_{0}$. We use the same random variables as before to update this second configuration, with one essential difference. In the second configuration, we prohibit the opening of an edge if this opening creates a connection between the top $T$ and the bottom $B$. This mechanism ensures that $X_{t}$ is always above $Y_{t}$. Moreover, a classical result on reversible Markov chains ensures that the invariant probability measure of the process $\left(Y_{t}\right)_{t \in \mathbb{N}}$ is the conditional probability measure $P_{p}(\cdot \mid T \longleftrightarrow B)$. Our coupling probability measure $\mu_{p}$ is defined as the invariant probability measure of the process $\left(X_{t}, Y_{t}\right)_{t \in \mathbb{N}}$. In the case of the Ising model, where one has access to an explicit formula for the equilibrium measure, one usually derives results on the dynamics (for instance the Glauber dynamics) from results on the Ising Gibbs measure. We go here in the reverse direction: we use our dynamical construction to derive results on the equilibrium measure $\mu_{p}$. Along the way, we obtain several results on the evolution of the pivotal edges in the dynamical percolation process, which are of independent interest, and which do not rely on equilibrium results (see Section 4).

For the proof, we consider the stationary process $\left(X_{t}, Y_{t}\right)_{t \in \mathbb{N}}$ starting from its equilibrium distribution $\mu_{p}$. We fix a time $t$ and we estimate the probability that the configuration $\left(X_{t}, Y_{t}\right)$ realizes the event appearing in the statement of Theorem 1.1. We distinguish the case of edges in the interface which are pivotal or not. For pivotal edges, we shall prove the following slightly stronger result.

Proposition 1.4. There exists $\tilde{p}<1$ and $\kappa>1$ such that, for $p \geqslant \tilde{p}$, and for any $c \geqslant 1$ and any box $\Lambda$ satisfying $|\Lambda|>3^{6 d}$, we have

$$
P_{p}\left(\exists e \in \mathcal{P}, d\left(e, \Lambda^{c} \cup \mathcal{P} \backslash\{e\}\right) \geqslant \kappa c \ln |\Lambda| \mid T \longleftrightarrow B\right) \leqslant \frac{1}{|\Lambda|^{c}} .
$$

The proof of this proposition relies on the BK inequality. We consider next the case of an edge $e$ in the interface which is not pivotal. Such an edge $e$ can be opened at any time in the configuration $Y$. Therefore, unless it becomes pivotal again, it cannot stay for a long time in the interface. In addition, before becoming part of the interface $\mathcal{I}$, the edge $e$ must have been pivotal. Indeed, nonpivotal edges in the process $\left(Y_{t}\right)_{t \in \mathbb{N}}$ evolve exactly as in the process $\left(X_{t}\right)_{t \in \mathbb{N}}$. We look backwards in the past at the last time when the edge $e$ was still pivotal. As said before, this time must be quite close from $t$. However, at time $t$, it turns out that the set of the pivotal edges is quite far from $e$. We conclude that the set of the pivotal edges must have moved away from $e$ very fast. To estimate the probability of a fast movement of the set $\mathcal{P}$, we derive an estimate on the speed of the set of the pivotal edges, which is stated in Proposition 4.1. This estimate is at the heart of the argument. It relies on the construction of specific space-time paths, which describe how the influence of the conditioning propagates in the box. If a space-time path travels over a long distance in a short time, then this implies that a certain sequence of closing events has occurred, and we estimate the corresponding probability. This estimate is delicate, because the closed space-time path can take 
advantage of the pivotal edges which remain closed thanks to the conditioning. The computation relies again on the BK inequality, this time applied to the space-time paths.

The statement of Theorem 1.1 naturally prompts several questions. First, the results presented here hold only for values of $p$ sufficiently close to 1 , because the proofs rely on Peierls arguments.

Question 1. Is it possible to prove an analogous result throughout the supercritical regime $p>p_{c}$ ?

Proposition 1.4 shows that, typically, each pivotal edge is within a distance of order $\ln |\Lambda|$ of another pivotal edge. Of course, we would like to understand better the random set $\mathcal{P}$.

Question 2. What else can be said about the structure of the set $\mathcal{P}$ ?

This question is essential to understand the fluctuation of the interfaces. For $p$ close to 1, and for boxes whose sides are parallel to the axis, it follows from Gielis and Grimmett (2002) (which is the percolation analog of Dobrushin's result, see Dobrushin, 1972) that the set $\mathcal{P}$ is flat with exponentially bounded local fluctuations. In this paper, we present a partial result on the speed of the pivotal edges.

Theorem 1.5. There exists $\tilde{p}<1$ such that for $p \geqslant \tilde{p}, c \geqslant 1$ and any box $\Lambda$ satisfying $|\Lambda| \geqslant e^{2 d^{2} c}$, we have

$$
P_{\mu}\left(d_{H}^{2 d c \ln |\Lambda|}\left(\bigcup_{r \in[t-c|\Lambda| \ln \Lambda, t]} \mathcal{P}_{r}, \bigcup_{s \in[t, t+c|\Lambda| \ln \Lambda]} \mathcal{P}_{s}\right) \geqslant 2 d c \ln |\Lambda|\right) \leqslant \frac{1}{|\Lambda|^{c-3}} .
$$

To control the distance between pivotal edges at two different times, we need to control the speed of the pivotal edges. In order to prove Theorem 1.5, we control the speed of these edges during a time interval of order $|\Lambda|$. When we study the pivotal edges on a time interval of length $2 d c|\Lambda| \ln |\Lambda|$, the previous results give us a control of the distance of order $\ln ^{2}|\Lambda|$ instead of $\ln |\Lambda|$. To remove the square, we need a new ingredient compared to the previous argument. Here we obtain a speed estimate on a time interval of order $|\Lambda| \ln |\Lambda|$ by studying a new type of space-time path which connects a pivotal edge at time $t$ to an edge of $\mathcal{P}_{s} \cup \mathcal{I}_{s}$ at a time $s<t$. The length of this new type of space-time paths has an exponential decay property during a time interval of order $|\Lambda| \ln |\Lambda|$. As a drawback, we have to replace $\mathcal{P}$ by $\mathcal{P} \cup \mathcal{I}$ due to the construction of this new space-time path. As a consequence, we can only study the distance between a pivotal edge and the union of the pivotal edges on a time interval in the past.

Question 3. Is it possible to replace $(\ln |\Lambda|)^{2}$ by $\ln |\Lambda|$ in the statement of Theorem 1.1?

Since there is no square in the logarithm appearing in Proposition 1.4, we suspect that it should also be the case in the statement of Theorem 1.1. Ultimately, we would like to gain some understanding on the Ising interfaces. The natural road to transfer percolation results towards the Ising model is to use the FK percolation model. However, there are several difficulties to overcome in order to adapt the proof to FK percolation. First, we use the BK inequality twice in the proof, and this inequality is not available in the FK model. Second, the dynamics for the FK model is more complicated.

Question 4. Does Theorem 1.1 extend to the FK percolation model?

Suppose that the answer to question 4 is positive. It is not obvious to transcribe Theorem 1.1 in the Ising context. For instance, the pivotal edges, which can be detected by visual inspection of a percolation configuration, are hidden inside the associated Ising configuration.

Question 5. What is the counterpart of Theorem 1.1 for Ising interfaces?

We hope to attack successfully these questions in future works. The question 4 and 5 are tackled in Zhou (2019).

The paper is organized as follows. In Section 2, we define precisely the model and the notations. Beyond the classical percolation definitions, this section contains the definition of the space-time 
paths and the graphical construction of the coupling. Section 3 is devoted to the proof of Proposition 1.4. In Section 4, we prove the central result on the control of the speed of the set of the pivotal edges. Then, the Theorem 1.1 is proved in Section 5. In Section 6, we improve the results obtained in Section 4 and we prove the Theorem 1.3 in Section 7. Then we continue our study to prove Theorem 1.5. In Section 8, we construct the new space-time path which will be used in the proof. In section 9, we control the distance between $\mathcal{P}_{t} \cup \mathcal{I}_{t}$ and $\mathcal{P}_{t+s}$ for $s \leqslant|\Lambda| \ln |\Lambda|$ with the help of this space-time path. Finally, the proof of Theorem 1.5 is presented in the Section 10.

\section{The model and notations}

2.1. Geometric definitions. We give standard geometric definitions.

The edges $\mathbb{E}^{d}$. The set of edges $\mathbb{E}^{d}$ is the set of the pairs $\{x, y\}$ of points of $\mathbb{Z}^{d}$ which are at Euclidean distance 1.

The box $\Lambda$. We will mostly work in a closed box $\Lambda$ centred at the origin. We denote by $T$ the top side of $\Lambda$ and by $B$ its bottom side.

The separating sets. Let $A, B$ be two subsets of $\Lambda$. We say that a set of edges $S \subset \Lambda$ separates $A$ and $B$ if no connected subset of $\Lambda \cap \mathbb{E}^{d} \backslash S$ intersects both $A$ and $B$. Such a set $S$ is called a separating set for $A$ and $B$. We say that a separating set is minimal if there does not exist a strict subset of $S$ which separates $A$ and $B$.

The cuts. We say that $S$ is a cut if $S$ separates $T$ and $B$, and $S$ is minimal for the inclusion.

The usual paths. We say that two edges $e$ and $f$ are neighbours if they have one endpoint in common. A usual path is a sequence of edges $\left(e, \ldots, e_{n}\right)$ such that for $1 \leqslant i<n$, the edge $e_{i}$ and $e_{i+1}$ are neighbours.

The $*$-paths. In order to study the cuts in any dimension $d \geqslant 2$, we use $*$-connectedness on the edges as in Deuschel and Pisztora (1996). We consider the supremum norm on $\mathbb{R}^{d}$ :

$$
\forall x=\left(x_{1}, \ldots, x_{d}\right) \in \mathbb{R}^{d} \quad\|x\|_{\infty}=\max _{i=1, \ldots, d}\left|x_{i}\right| .
$$

For $e$ an edge in $\mathbb{E}^{d}$, we denote by $m_{e}$ the center of the unit segment associated to $e$. We say that two edges $e$ and $f$ of $\mathbb{E}^{d}$ are $*$-neighbours if $\left\|m_{e}-m_{f}\right\|_{\infty} \leqslant 1$. A $*$-path is a sequence of edges $\left(e_{1}, \ldots, e_{n}\right)$ such that, for $1 \leqslant i<n$, the edge $e_{i}$ and $e_{i+1}$ are $*$-neighbours.

2.2. The dynamical percolation. We define the dynamical percolation and the space-time paths.

Percolation configurations. A percolation configuration in $\Lambda$ is a map from the set of the edges included in $\Lambda$ to $\{0,1\}$. An edge $e \subset \Lambda$ is said to be open in a configuration $\omega$ if $\omega(e)=1$ and closed if $\omega(e)=0$. For two subsets $A, B$ of $\Lambda$ and a configuration $\omega \in \Omega$, we denote by $A \stackrel{\omega}{\longleftrightarrow} B$ the event that there is an open path between a vertex of $A$ and a vertex of $B$ in the configuration $\omega$.

Probability measures. We denote by $P_{p}$ the law of the Bernoulli bond percolation in the box $\Lambda$ with parameter $p$. The probability $P_{p}$ is the probability measure on the set of bond configurations which is the product of the Bernoulli distribution $(1-p) \delta_{0}+p \delta_{1}$ over the edges included in $\Lambda$. We define $P_{\mathcal{D}}$ as the probability measure $P_{p}$ conditioned on the event $\{T \leftrightarrow B\}$, i.e.,

$$
P_{\mathcal{D}}(\cdot)=P_{p}(\cdot \mid T \longleftrightarrow B) .
$$

Probability space. Throughout the paper, we assume that all the random variables used in the proofs are defined on the same probability space $\Omega$. For instance, this space contains the random variables used in the graphical construction presented below, as well as the random variables generating the initial configurations of the Markov chains. We denote simply by $P$ the probability measure on $\Omega$. 
Graphical construction. We now present a graphical construction of the dynamical percolation in the box $\Lambda$. This graphical construction is a discrete time analog of the graphical construction introduced by Schonmann to study the Glauber dynamics of the Ising model (see Schonmann, 1994). We build a sequence of triplets $\left(X_{t}, E_{t}, B_{t}\right)_{t \in \mathbb{N}}$, where $X_{t}$ is the percolation configuration in $\Lambda$ at time $t, E_{t}$ is a random edge in the box $\Lambda$ and $B_{t}$ is a Bernoulli random variable. The sequence $\left(E_{t}\right)_{t \in \mathbb{N}}$ is an i.i.d. sequence of edges, with uniform distribution over the edges included in $\Lambda$. The sequence $\left(B_{t}\right)_{t \in \mathbb{N}}$ is an i.i.d. sequence of Bernoulli random variables with parameter $p$. The sequence $\left(E_{t}\right)_{t \in \mathbb{N}}$ and $\left(B_{t}\right)_{t \in \mathbb{N}}$ are independent. The process $\left(X_{t}\right)_{t \in \mathbb{N}}$ is built iteratively as follows. At time 0 , we start from the configuration $X_{0}$, which might be random. At time $t$, we change the state of $E_{t}$ to $B_{t}$ and we set

$$
\forall t \geqslant 1 \quad X_{t}(e)=\left\{\begin{array}{cc}
X_{t-1}(e) & \text { if } E_{t} \neq e \\
B_{t} & \text { if } E_{t}=e
\end{array} .\right.
$$

The process $\left(X_{t}\right)_{t \in \mathbb{N}}$ is the dynamical percolation process in the box $\Lambda$.

The space-time paths. We introduce the space-time paths which generalise both the usual paths and the $*$-paths to the dynamical percolation. A space-time path is a sequence of pairs, called time-edges, $\left(e_{i}, t_{i}\right)_{1 \leqslant i \leqslant n}$, such that, for $1 \leqslant i \leqslant n-1$, we have either $e_{i}=e_{i+1}$, or $\left(e_{i}, e_{i+1}\right.$ are neighbours and $\left.t_{i}=t_{i+1}\right)$. We say that a space-time path $\left(e_{i}, t_{i}\right)_{1 \leqslant i \leqslant n}$ is during a time interval $[s, t]$ if for all $1 \leqslant i \leqslant n$, we have $t_{i} \in[s, t]$. We define also space-time $*$-paths, by using edges which are $*$-neighbours in the above definition. For $s, t$ two integers, we define

$$
s \wedge t=\min (s, t), \quad s \vee t=\max (s, t) .
$$

A space-time path $\left(e_{i}, t_{i}\right)_{1 \leqslant i \leqslant n}$ is open in the dynamical percolation process $\left(X_{t}\right)_{t \in \mathbb{N}}$ if

$$
\forall i \in\{1, \ldots, n\} \quad X_{t_{i}}\left(e_{i}\right)=1
$$

and

$$
\forall i \in\{1, \ldots, n-1\} \quad e_{i}=e_{i+1} \quad \Longrightarrow \quad \forall t \in\left[t_{i} \wedge t_{i+1}, t_{i} \vee t_{i+1}\right] \quad X_{t}\left(e_{i}\right)=1 .
$$

In the same way, we can define a closed space-time path by changing 1 to 0 in the previous definition. In the remaining of the article, we use the abbreviation STP to design a space-time path. Moreover, unless otherwise specified, the closed paths (and the closed STPs) are defined with the relation $*$ and the open paths (and the open STPs) are defined with the usual relation. This is because the closed paths come from the cuts, while the open paths come from existing connexions.

2.3. The interfaces by coupling. We propose a new way of defining the interfaces by coupling two processes of dynamical percolation. We start with the graphical construction $\left(X_{t}, E_{t}, B_{t}\right)_{t \in \mathbb{N}}$ of the dynamical percolation. We define a further process $\left(Y_{t}\right)_{t \in \mathbb{N}}$ as follows: at time 0 , we set $X_{0}=Y_{0}$, and for all $t \geqslant 1$, we set

$$
\forall e \subset \Lambda \quad Y_{t}(e)=\left\{\begin{array}{ccl}
Y_{t-1}(e) & \text { if } \quad e \neq E_{t} \\
0 & \text { if } \quad e=E_{t} \text { and } B_{t}=0 \\
1 & \text { if } \quad e=E_{t}, B_{t}=1 \text { and } T \stackrel{Y_{t-1}^{E_{t}}}{\leftrightarrows} B \\
0 & \text { if } \quad e=E_{t}, B_{t}=1 \text { and } T \stackrel{Y_{t-1}^{E_{t}}}{\longleftrightarrow} B
\end{array},\right.
$$

where, for a configuration $\omega$ and an edge $e$, the notation $\omega^{e}$ means the configuration obtained by opening $e$ in $\omega$. Typically, we start with a configuration $Y_{0}$ realizing the event $\{T \leftrightarrow B\}$, but this is not mandatory in the above definition. An illustration of this dynamics is given in the Figure 2.1. We denote by $P_{\mathcal{D}}$ the equilibrium distribution of the process $\left(Y_{t}\right)_{t \in \mathbb{N}}$. Before opening a closed edge $e$ at time $t$, we verify whether this will create a connexion between $T$ and $B$. If it is the case, the edge $e$ stays closed in the process $\left(Y_{t}\right)_{t \in \mathbb{N}}$ but can be opened in the process $\left(X_{t}\right)_{t \in \mathbb{N}}$, otherwise the edge $e$ is opened in both processes $\left(X_{t}\right)_{t \in \mathbb{N}}$ and $\left(Y_{t}\right)_{t \in \mathbb{N}}$. On the contrary, the two processes 

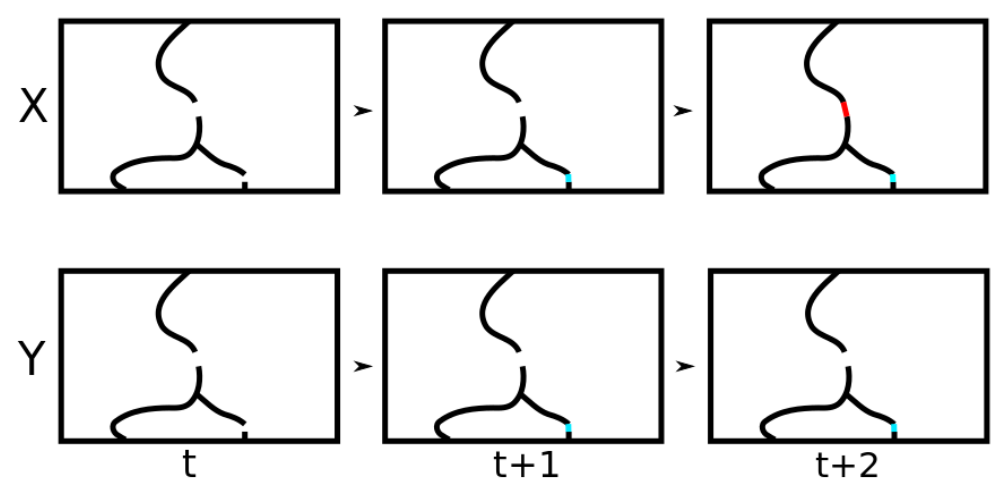

Figure 2.1. A coupling of the process $\left(X_{t}, Y_{t}\right)_{t \in \mathbb{N}}$. At time $t+1$ we try to open the blue edge and at time $t+2$, we try to open the red edge.

behave similarly for the edge closing events since we cannot create a new connexion by closing an edge. The set of the configurations satisfying $\{T \longleftrightarrow B\}$ is irreducible and the process $\left(X_{t}\right)_{t \in \mathbb{N}}$ is reversible. Therefore, the process $\left(Y_{t}\right)_{t \in \mathbb{N}}$ is the dynamical percolation conditioned to satisfy the event $\{T \longleftrightarrow B\}$. According to the Lemma 1.9 of Kelly (2011), the invariant probability measure of $\left(Y_{t}\right)_{t \in \mathbb{N}}$ is $P_{\mathcal{D}}$, the probability $P_{p}$ conditioned by the event $\{T \leftrightarrow B\}$, i.e.,

$$
P_{\mathcal{D}}(\cdot)=P_{p}(\cdot \mid T \leftrightarrow B) .
$$

Suppose that we start from a configuration $\left(X_{0}, Y_{0}\right)$ belonging to the set

$$
\mathcal{E}=\left\{\left(\omega_{1}, \omega_{2}\right) \in\{0,1\}^{\mathbb{E}^{d} \cap \Lambda} \times\{T \longleftrightarrow B\}: \forall e \subset \Lambda \quad \omega_{1}(e) \geqslant \omega_{2}(e)\right\} .
$$

The set $\mathcal{E}$ is irreducible and aperiodic. In fact, each configuration of $\mathcal{E}$ communicates with the configuration where all edges are closed. The state space $\mathcal{E}$ is finite, therefore the Markov chain $\left(X_{t}, Y_{t}\right)_{t \in \mathbb{N}}$ admits a unique equilibrium distribution $\mu_{p}$. We denote by $P_{\mu}$ the law of the process $\left(X_{t}, Y_{t}\right)_{t \in \mathbb{N}}$ starting from a random initial configuration $\left(X_{0}, Y_{0}\right)$ with distribution $\mu_{p}$. We now present a definition of the interface between $T$ and $B$ based on the previous coupling.

Definition 2.1. The interface at time $t$ between $T$ and $B$, denoted by $\mathcal{I}_{t}$, is the set of the edges in $\Lambda$ that differ in the configurations $X_{t}$ and $Y_{t}$, i.e.,

$$
\mathcal{I}_{t}=\left\{e \subset \Lambda: X_{t}(e) \neq Y_{t}(e)\right\} \text {. }
$$

The edges of $\mathcal{I}_{t}$ are open in $X_{t}$ but closed in $Y_{t}$ and the configuration $X_{t}$ is above the configuration $Y_{t}$. We define next the set $\mathcal{P}_{t}$ of the pivotal edges for the event $\left\{T \leftrightarrow \leftrightarrow B\right.$ in the configuration $Y_{t}$.

Definition 2.2. The set $\mathcal{P}_{t}$ of the pivotal edges in $Y_{t}$ is the collection of the edges in $\Lambda$ whose opening would create a connection between $T$ and $B$, i.e.,

$$
\mathcal{P}_{t}=\left\{e \subset \Lambda: T \stackrel{Y_{t}^{e}}{\longleftrightarrow} B\right\} .
$$

We define finally the set $\mathcal{C}_{t}$ of the cuts in $Y_{t}$.

Definition 2.3. The set $\mathcal{C}_{t}$ of the cuts in $Y_{t}$ is the collection of the cuts in $\Lambda$ at time $t$.

\section{The isolated pivotal edges}

In this section, we will show the Proposition 1.4. We first investigate the structure of the set of the cuts. In a configuration $\omega$ realizing the event $\{T \longleftrightarrow \leftrightarrow B\}$, we will identify two separating sets $S^{+}$and $S^{-}$. We construct $S^{+}$by considering the open cluster

$$
O(T)=\left\{x \in \mathbb{Z}^{d} \cap \Lambda: x \stackrel{\omega}{\longleftrightarrow} T\right\} .
$$


We consider the set $O(T)^{c}=\mathbb{Z}^{d} \backslash O(T)$. As $\mathbb{Z}^{d} \backslash \Lambda$ is $*$-connected, there exists only finitely many *-connected components of $O(T)^{c}$ and exactly one of them is of infinite size. We denote these components by $G, H_{1}, \ldots, H_{k}$ where $G$ is the unique infinite component. We set

$$
O^{\prime}(T)=O(T) \cup H_{1} \cup \cdots \cup H_{k} .
$$

The set $O^{\prime}(T)$ is $*$-connected and has no holes. For a $*$-connected set $A \subset \mathbb{Z}^{d}$, we define the external boundary of $A$, denoted by $\partial^{e x t} A$, as

$$
\partial^{e x t} A=\left\{\{x, y\} \in \mathbb{E}^{d}: x \in A, y \notin A\right\} .
$$

We then define $S^{+}$as the subset of $\partial^{e x t} O^{\prime}(T)$ consisting of the edges of $\partial^{e x t} O^{\prime}(T)$ which are included in $\Lambda$. In a similar way, we define $S^{-}$by replacing $T$ by $B$ in the previous construction. Each of the two sets contains a cut. An illustration of these two separating sets can be found in the Figure 3.2.

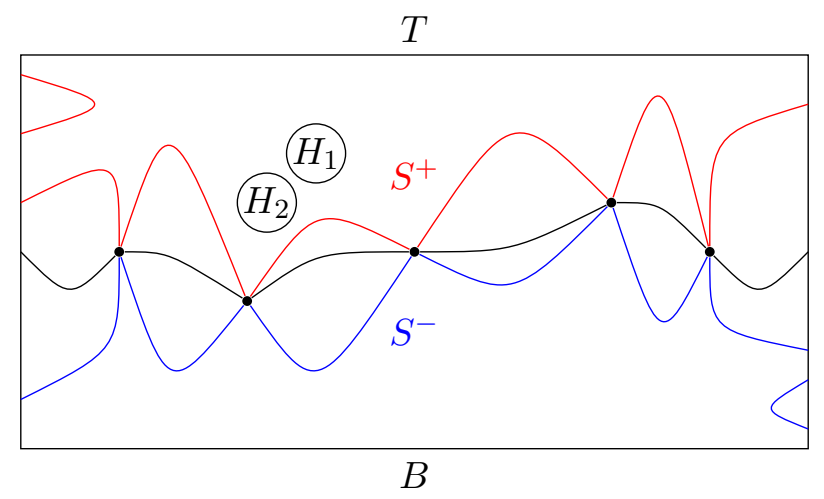

Figure 3.2. The sets $S^{+}$(red) and the set $S^{-}$(blue).

Lemma 3.1. The sets $\partial^{\text {ext }} O^{\prime}(T)$ and $\partial^{\text {ext }} O^{\prime}(B)$ are $*$-connected.

This result is a direct consequence of the first point in Lemma 2.1 in Deuschel and Pisztora (1996). We also mention the Lemma 2.23 in Kesten (1986) for a similar result on the set of vertices and a shorter argument presented in Timár (2013). We explain next the relation between the sets $S^{+}$, $S^{-}$and $\mathcal{P}$.

Lemma 3.2. The set $\mathcal{P}$ of the pivotal edges is the intersection between $S^{+}$and $S^{-}$.

Proof: We have the inclusion $\mathcal{P} \subset S^{+} \cap S^{-}$since all the pivotal edges are in all the cuts. Both $S^{+}$ and $S^{-}$contain a cut. We consider next an edge $e$ in $S^{+} \cap S^{-}$. Since $S^{+}$consists of the boundary edges of $O(T)$, there is an open path between $T$ and $e$. The same result holds for $S^{-}$. Therefore, there is a path between $T$ and $B$ whose edges other than $e$ are open. By opening $e$, we realise the event $\{T \longleftrightarrow B\}$. In other words, the edge $e$ is included in $\mathcal{P}$. We conclude that $S^{+} \cap S^{-} \subset \mathcal{P}$.

We also need a combinatorial result on the $*$-connectedness in dimension $d$.

Lemma 3.3. In the d-dimensional lattice, the number of $*$-neighbours of an edge $e$ is

$$
\alpha(d)=3^{d}+4(d-1) 3^{d-2}-1 .
$$

Proof: An $*$-neighbour edge $f$ of $e$ is either parallel to $e$ or belongs to the $(d-1)$-cube centred at a vertex of $e$ and of side-length 2 perpendicular to $e$. For the edges that are parallel to $e$, the distance between their centres is 1 and there are $3^{d}-1$ such edges. A $(d-1)$-cube of side-length 2 has $2(d-1) 3^{d-2}$ edges. Hence there are $3^{d}+4(d-1) 3^{d-2}-1 *$-neighbours of $e$. 
We now prove the Proposition 1.4. The main idea of the proof is to observe a long closed path outside of a cut whenever a pivotal edge is isolated. We use then the BK inequality and we conclude with the help of classical arguments of exponential decay.

Proof of Proposition 1.4: Since there is a pivotal edge which is at distance more than 1 from the others, there is a cut which contains at least one non pivotal edge. By Lemma 3.2, this cut is not included in $S^{-} \cap S^{+}$, thus there are at least two distinct cuts in the configuration. Let $e$ be an edge of $\mathcal{P}$ which is at distance at least $\kappa c \ln |\Lambda|$ from $\Lambda^{c} \cup \mathcal{P} \backslash\{e\}$. Let $e^{\prime}$ be the pivotal edge which is nearest to $e$ or one of them if there are several. By Lemma 3.1, there is a closed $*$-path included in $\partial^{e x t} O(T)$ between $e$ and $e^{\prime}$. This path might exit from the box $\Lambda$, since $\partial^{e x t} O(T)$ is defined as the external boundary of $O^{\prime}(T)$, where $O^{\prime}(T)$ is seen as a subset of $\mathbb{Z}^{d}$, not of $\Lambda$. However, since $e$ is at distance at least $\kappa c \ln |\Lambda|$ from $\mathcal{P} \backslash\{e\}$ and from $\Lambda^{c}$, the initial portion of the closed $*$-path from its origin until it has travelled a distance $\kappa c \ln |\Lambda|$ is inside the box $\Lambda$, it consists of closed edges which are not pivotal, and therefore, by Lemma 3.2, it is also disjoint from the set $S^{-}$. Let us denote by $\mathcal{E}(e)$ the event:

$$
\mathcal{E}(e)=\left\{\begin{array}{c}
\text { there exists a closed } * \text {-path starting at one } * \text {-neighbour of } e \\
\text { which travels a distance at least } \kappa c \ln |\Lambda|-2 d
\end{array}\right\} .
$$

From the previous discussion, we conclude that

$$
\left\{e \in \mathcal{P}, d\left(e, \Lambda^{c} \cup \mathcal{P} \backslash\{e\}\right) \geqslant \kappa c \ln |\Lambda|\right\} \cap\{T \longleftrightarrow B\} \quad \subset \quad \mathcal{E}(e) \circ\{T \longleftrightarrow B\},
$$

where $\circ$ means the disjoint occurrence. Therefore, we have the following inequality:

$$
P_{\mathcal{D}}\left(e \in \mathcal{P}, d\left(e, \Lambda^{c} \cup \mathcal{P} \backslash\{e\}\right) \geqslant \kappa c \ln |\Lambda|\right) \leqslant P_{\mathcal{D}}(\mathcal{E}(e) \circ\{T \longleftrightarrow B\}) .
$$

By the definition of $P_{\mathcal{D}}$, we have

$$
P_{\mathcal{D}}(\mathcal{E}(e) \circ\{T \leftrightarrow B\})=\frac{P_{p}(\mathcal{E}(e) \circ\{T \leftrightarrow B\})}{P_{p}(T \longleftrightarrow B)} .
$$

Note that the event $\mathcal{E}(e)$ and $\{T \leftrightarrow B\}$ are both decreasing. Applying the BK inequality (see Grimmett, 1999), we get

$$
P_{\mathcal{D}}\left(e \in \mathcal{P}, d\left(e, \Lambda^{c} \cup \mathcal{P} \backslash\{e\}\right) \geqslant \kappa c \ln |\Lambda|\right) \leqslant P_{p}(\mathcal{E}(e)) .
$$

The closed $*$-path in the event $\mathcal{E}(e)$ starts at a neighbour of $e$ and travels a distance at least $\kappa c \ln |\Lambda|-2 d$. By this, we mean that there is an Euclidean distance at least $\kappa c \ln |\Lambda|-2 d$ from one endpoint of the first edge of the path to one endpoint of the last edge of the path. The distance between the centres of two *-neighbouring edges is at most $d$, therefore the number of edges in such a path is at least

$$
\frac{1}{d}(\kappa c \ln |\Lambda|-2 d-1) .
$$

We assume that $|\Lambda| \geqslant 3^{6 d}$ and we choose $\kappa>1$, whence, for $c \geqslant 1$,

$$
\kappa c \ln |\Lambda|-2 d-1 \geqslant \frac{\kappa c}{2} \ln |\Lambda| .
$$

Hence

$$
P_{p}(\mathcal{E}(e)) \leqslant(1-p)^{\frac{\kappa c}{2 d} \ln |\Lambda|} \alpha(d) \frac{\kappa c}{2 d} \ln |\Lambda|
$$

We then sum the probability over all the edges $e$ in $\Lambda$. We obtain

$$
P_{\mathcal{D}}\left(\exists e \in \mathcal{P}, d\left(e, \Lambda^{c} \cup \mathcal{P} \backslash\{e\}\right) \geqslant \kappa c \ln |\Lambda|\right) \leqslant d|\Lambda|^{1+\frac{\kappa c}{2 d} \ln ((1-p) \alpha(d)) .}
$$


We choose $\tilde{p}<1$ such that $(1-\tilde{p}) \alpha(d)<1$. There exists a $\kappa>0$ such that, for any $p \geqslant \tilde{p}$ and any $c>0$, we have

$$
d|\Lambda|^{1+\frac{\kappa c}{2 d} \ln ((1-p) \alpha(d))} \leqslant \frac{1}{|\Lambda|^{c}},
$$

and we obtain the desired inequality.

We state now a corollary of the Proposition 1.4 which controls the distance between any cut present in the configuration and the set $\mathcal{P}$.

Corollary 3.4. There exists $\tilde{p}<1$ and $\kappa>1$ such that, for $p \geqslant \tilde{p}$, for any constant $c \geqslant 1$ and any box $\Lambda$ satisfying $|\Lambda|>3^{6 d}$, the following inequality holds:

$$
P_{\mathcal{D}}\left(\exists C \in \mathcal{C}, \exists e \in C, d\left(e, \mathcal{P} \cup \Lambda^{c}\right) \geqslant \kappa c \ln |\Lambda|\right) \leqslant \frac{1}{|\Lambda|^{c}} .
$$

Proof: Let $C$ be a cut and let $e$ be an edge of $C$ such that $d\left(e, \mathcal{P} \cup \Lambda^{c}\right) \geqslant \kappa c \ln |\Lambda|$. There exists a closed $*$-path included in $C$ which connects $e$ to a pivotal edge $f$. Within a distance less than $\kappa c \ln |\Lambda|$ from $e$, there is no pivotal edge. By stopping the path at the first pivotal edge that it encounters or at the first edge intersecting the boundary of $\Lambda$, we obtain a path $\left(e_{1}, \ldots, e_{n}\right)$ without pivotal edge. Suppose that this path encounters the set $S^{+}$or the set $S^{-}$. Let $e_{j}$ be the first edge of the path which is in $S^{+} \cup S^{-}$. By Lemma 3.2, the edge $e_{j}$ doesn't belong to $S^{+} \cap S^{-}$. Without loss of generality, we can suppose that $e_{j} \in S^{+} \backslash S^{-}$. We concatenate $\left(e_{1}, \ldots, e_{j}\right)$ and a closed path in $\partial^{e x t} O^{\prime}(T)$ from $e_{j}$ to a pivotal edge or to an edge on the boundary of $\Lambda$. We obtain a closed path disjoint from $S^{-}$. We reuse the same techniques as in the proof of 1.4 and we obtain the desired result.

We shall also study the case where there is no pivotal edge in a configuration.

Proposition 3.5. There exists a constant $\tilde{p}<1$, such that,

$$
\forall p \geqslant \tilde{p} \quad \forall \Lambda \quad P_{\mathcal{D}}(\mathcal{P}=\emptyset) \leqslant d|\Lambda| \exp (-D),
$$

where $D$ is the diameter of $T$ (or $B)$.

Proof: Suppose that $\mathcal{P}$ is empty. By Lemma 3.2, the set $S^{+}$and the set $S^{-}$are then disjoint. Each of them contains a cut. Therefore, there are two disjoint closed $*$-paths travelling a distance at least $|\Lambda|^{1 / d}$. By the same reasoning as in the proof of Proposition 1.4, the $P_{\mathcal{D}}$ probability of this event can be bounded by

$$
P_{p}(\exists \gamma \text { closed path } \subset \Lambda, \gamma \text { travels a distance at least } D) .
$$

Since there are at least $D / d$ edges in such a path $\gamma$, this probability is less than

$$
d|\Lambda|(\alpha(d)(1-p)) \frac{D}{d}
$$

There exists $\tilde{p}<1$ such that, for all $\Lambda$, we have

$$
\forall p \geqslant \tilde{p} \quad d|\Lambda|(\alpha(d)(1-p))^{\frac{D}{d}} \leqslant d|\Lambda| \exp (-D) .
$$

This yields the desired inequality. 


\section{Speed of the cuts}

We state now the crucial proposition which gives a control on the speed of the cuts.

Proposition 4.1. There exists $\tilde{p}<1$, such that for $p \geqslant \tilde{p}$, for any $\ell \geqslant 2, t \in \mathbb{N}, s \in\{0, \ldots,|\Lambda|\}$ and any edge $e \subset \Lambda$ at distance more than $\ell$ from $\Lambda^{c}$,

$$
P_{\mu}\left(\begin{array}{c}
e \in \mathcal{P}_{t+s} \\
\forall r \in[t, t+s] \quad \mathcal{P}_{r} \neq \emptyset
\end{array} \mid \exists c_{t} \in \mathcal{C}_{t}, d\left(e, c_{t}\right) \geqslant \ell\right) \leqslant \exp (-\ell) .
$$

To prove this result, we will construct a STP associated to the movement of the pivotal edges and then show that the probability to have such a long STP decreases exponentially fast as the length of the path grows.

4.1. Construction of the STP. We start by defining some properties of a STP. In the rest of the paper, unless otherwise specified, all the closed paths (and the closed STPs) are defined with the relation $*$ and the open paths (and the open STPs) are defined with the usual relation.

Definition 4.2. A STP $\left(e_{1}, t_{1}\right), \ldots,\left(e_{n}, t_{n}\right)$ is increasing (respectively decreasing) if

$$
t_{1} \leqslant \cdots \leqslant t_{n}\left(\text { resp. } t_{1} \geqslant \cdots \geqslant t_{n}\right) .
$$

If a STP is increasing or decreasing, we say that it is monotone.

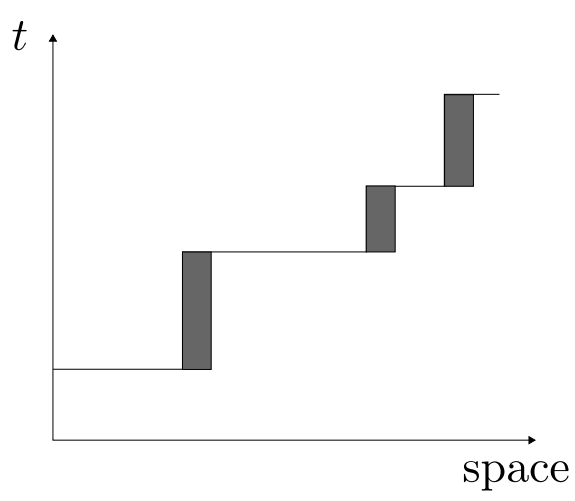

Figure 4.3. An increasing STP with its time change intervals in gray

Definition 4.3. A closed STP $\left(e_{1}, t_{1}\right), \ldots,\left(e_{n}, t_{n}\right)$ in $X$ (respectively $Y$ ) is called simple if each edge is visited only once or it is opened at least once between any two consecutive visits, i.e., for any $i, j$ in $\{1, \ldots, n\}$ such that $|i-j| \neq 1$,

$$
\left.\left.\left(e_{i}=e_{j} \quad t_{i}<t_{j}\right) \quad \Longrightarrow \quad \exists s \in\right] t_{i}, t_{j}\right] \quad X_{s}\left(e_{i}\right)=1 \quad\left(\text { resp. } Y_{s}\left(e_{i}\right)=1\right) .
$$

We show next that two pivotal edges occurring at different times are connected through a monotone simple STP closed in $Y$.

Proposition 4.4. Let $s$ and $t$ be two times such that $s<t$. We suppose that $\mathcal{P}_{r}$ is not empty for all $r \in[s, t]$. Let $f \in \mathcal{P}_{s}$ and $e \in \mathcal{P}_{t}$. Then there exists a decreasing simple STP $\gamma$ closed in $Y$ from $(e, t)$ to $(f, s)$ or a decreasing simple STP closed in $Y$ from $(e, t)$ to $(g, \alpha)$ where $g$ is an edge meeting the boundary $\partial \Lambda$ of $\Lambda$ and $\alpha \in[s, t]$. 


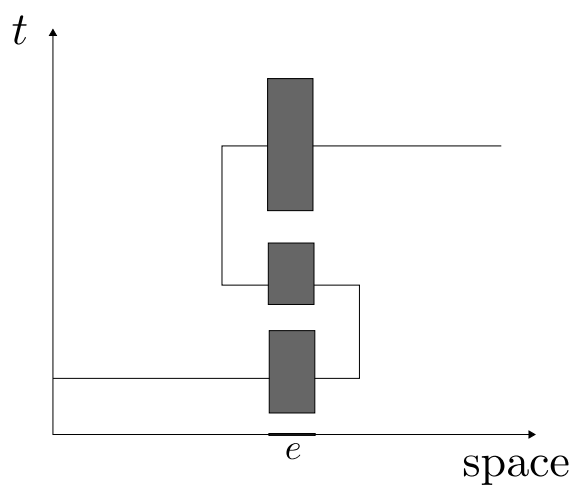

Figure 4.4. A simple STP, intervals of closure of the edge $e$ in gray

Proof: By Lemma 3.1, the edges of $\mathcal{P}_{t}$ are connected by a $*$-path which might possibly exit from $\Lambda$, but whose edges included in $\Lambda$ are closed in $Y_{t}$. We consider the function $\theta(t)$ giving the time when the oldest edge of $\mathcal{P}_{t}$ appeared, i.e.,

$$
\theta(t)=\min _{\varepsilon \in \mathcal{P}_{t}} \min \left\{r \leqslant t: \varepsilon \in \mathcal{P}_{r}, \varepsilon \notin \mathcal{P}_{r-1}, \forall \alpha \in[r, t], \varepsilon \in \mathcal{P}_{\alpha}\right\} .
$$

We denote by $e_{1}$ one of the edges realizing the minimum $\theta(t)$. We claim that $\theta(t)<t$. Indeed, suppose first that an edge closes at time $t$. Then a pivotal edge cannot be created at time $t$ and all the pivotal edges present at time $t$ were also pivotal at time $t-1$. Therefore $\theta(t) \leqslant t-1<t$. Suppose next that an edge opens at time $t$. Let us consider an edge $\varepsilon$ of $\mathcal{P}_{t-1}$, which is assumed to be not empty. At time $t-1$, there is one open path which connects $\varepsilon$ to $T$ and another one which connects $\varepsilon$ to $B$. Since one edge opens at time $t$, these two paths remain open at time $t$. Therefore $\varepsilon$ is still pivotal at time $t$. We have thus $\mathcal{P}_{t-1} \subset \mathcal{P}_{t}$ and it follows that $\theta(t) \leqslant t-1<t$. We have proved that $\theta(t)<t$.

If $\theta(t) \leqslant s$, we consider the STP obtained by connecting the path between $(e, t),\left(e_{1}, t\right)$ and the path between $\left(e_{1}, s\right),(f, s)$ with a time change from $t$ to $s$ on the edge $e_{1}$. If this STP does not encounter $\partial \Lambda$ then it answers the question. If it encounters $\partial \Lambda$, then we stop the STP at the first edge intersecting $\partial \Lambda$, we obtain a STP satisfying the second condition of the proposition. Suppose now that $\theta(t)>s$. We consider the edge $e_{1}$ at time $\theta(t)$. By construction, the edge $e_{1}$ belongs to $\mathcal{P}_{\theta(t)}$. Moreover, using Lemma 3.1, $\left(e_{1}, \theta(t)\right)$ is connected to $(e, t)$ by a STP consisting of a closed path at time $t$ and a time change from $t$ to $\theta(t)$ on the edge $e_{1}$. We take $\left(e_{1}, \theta(t)\right)$ as the new starting point. We repeat the procedure above and we obtain a sequence of times $\left(\theta(t), \theta(\theta(t)), \ldots, \theta^{(i)}(t), \ldots\right)$ by defining iteratively

$$
\theta^{i+1}(t)=\min _{\varepsilon \in \mathcal{P}_{\theta^{i}(t)}}\left\{r \leqslant \theta^{i}(t): \varepsilon \in \mathcal{P}_{r}, \varepsilon \notin \mathcal{P}_{r-1}, \forall \alpha \in\left[r, \theta^{i}(t)\right], \varepsilon \in \mathcal{P}_{\alpha}\right\} .
$$

For each index $i$, we choose an edge $e_{i} \in \mathcal{P}_{\theta^{i-1}(t)}$ which becomes pivotal at time $\theta^{(i)}(t)$. From the argument above, we obtain a strictly decreasing sequence

$$
t>\theta(t)>\cdots>\theta^{i}(t) .
$$

Therefore, there exists an index $k$ such that

$$
\theta^{k+1}(t) \leqslant s<\theta^{k}(t)
$$

For $i \in\{0, \ldots, k-1\}$, the edge-time $\left(e_{i}, \theta^{i}(t)\right)$ is connected to $\left(e_{i+1}, \theta^{i+1}(t)\right)$ by a decreasing STP $\gamma_{i}$ which is closed in $Y$. By concatenating these STPs, we obtain a decreasing STP between $(e, t)$ and $\left(e_{k}, \theta^{k}(t)\right)$. At time $\theta^{k}(t)$, there exists also a closed path $\rho$ between $e_{k}$ and $e_{k+1}$. We stop the 
time change at $s$ on the edge $e_{k+1}$ in order to arrive at an edge of $\mathcal{P}_{s}$. By Lemma 3.1, there is a closed path $\rho$ between $e_{k+1}$ and $f$ at time $s$. Therefore, the STP

$$
(e, t), \gamma_{0},\left(e_{1}, \theta(t)\right), \gamma_{1}, \ldots, \gamma_{k-1},\left(e_{k}, \theta^{k}(t)\right), \rho,\left(e_{k+1}, \theta^{k}(t)\right),\left(e_{k+1}, s\right), \rho,(f, s)
$$

is decreasing, closed in $Y$ and it connects $(e, t)$ and $(f, s)$. If this STP exits the box $\Lambda$, then the initial portion starting from $e$ until the first edge intersecting $\partial \Lambda$ satisfies the second condition of the proposition.

In order to obtain a STP which is simple in $Y$, we consider the following iterative procedure to modify a path. Let us denote by $\left(e_{i}, t_{i}\right)_{0 \leqslant i \leqslant N}$ the STP obtained previously. Starting with the edge $e_{0}$, we examine the rest of the edges one by one. Let $i \in\{0, \ldots, N\}$. Suppose that the edges $e_{0}, \ldots, e_{i-1}$ have been examined and let us focus on $e_{i}$. We encounter three cases:

- For every index $j \in\{i+1, \ldots, N\}$, we have $e_{j} \neq e_{i}$. Then, we don't modify anything and we start examining the edge $e_{i+1}$.

- There is an index $j \in\{i+1, \ldots, N\}$ such that $e_{i}=e_{j}$, but for the first index $k>i+1$ such that $e_{i}=e_{k}$, there is a time $\left.\alpha \in\right] t_{k}, t_{i}\left[\right.$ when $Y_{\alpha}\left(e_{i}\right)=1$. Then we don't modify anything and we start examining the next edge $e_{i+1}$.

- There is an index $j \in\{i+1, \ldots, N\}$ such that $e_{i}=e_{j}$ and for the first index $k>i+1$ such that $e_{i}=e_{k}$, we have $Y_{\alpha}\left(e_{i}\right)=0$ for all $\left.\alpha \in\right] t_{k}, t_{i}$. In this case, we remove all the time-edges whose indices are strictly between $i$ and $k$. We then have a simple time change between $t_{i}$ and $t_{k}$ on the edge $e_{i}$. We continue the procedure from the index $e_{k}$.

The STP becomes strictly shorter after every modification, and the procedure will end after a finite number of modifications. We obtain in the end a simple path in $Y$. Since the procedure doesn't change the order of the times $t_{i}$, we still have a decreasing path.

4.2. The BK inequality applied to a STP. Before embarking in technicalities, let us discuss the differences between the processes $\left(X_{t}\right)_{t \in \mathbb{N}}$ and $\left(Y_{t}\right)_{t \in \mathbb{N}}$. Let $(e, t)$ be a closed time-edge in $Y$. Since there is no constraint in the process $X$, the edge $e$ can be open in the configuration $X_{t}$. If the edge $e$ is open in $X_{t}$, then it belongs to $\mathcal{I}_{t}$. Now let us consider a time $t$ for which $E_{t}=e$ and $B_{t}=0$. Closing an edge doesn't create an open path between $T$ and $B$, thus the edge $e$ will be closed in both $X_{t}$ and $Y_{t}$. On the contrary, for a time $t$ such that $B_{t}=1$ and $E_{t}=e$ is pivotal at time $t-1$, the edge $e$ can be opened in the process $\left(X_{t}\right)_{t \in \mathbb{N}}$ but it remains closed in the process $\left(Y_{t}\right)_{t \in \mathbb{N}}$. Now let us consider the STP constructed in Proposition 4.4. Since the STP is closed in $Y$, each edge $e$ visited by the path is either closed at time $s$ or there is a time $r \in] s, t]$ when $E_{r}=e$ and $B_{r}=0$. In fact, since the STP is simple, then each edge is reopened and closed between two successive visits of the STP. Our first goal is to introduce the necessary notation in order to keep track of all the closing events implied by the STP.

We shall define the space projection of a STP. Given $k \in \mathbb{N}^{*}$ and a sequence $\Gamma=\left(e_{i}\right)_{1 \leqslant i \leqslant k}$ of edges, we say that it has length $k$, which we denote by length $(\Gamma)=k$, and we define its support

$$
\operatorname{support}(\Gamma)=\left\{e \subset \Lambda: \exists i \in\{1, \ldots, k\} \quad e_{i}=e\right\} .
$$

Let $\gamma=\left(e_{i}, t_{i}\right)_{1 \leqslant i \leqslant n}$ be a simple STP, the space projection of $\gamma$ is obtained by removing one edge in every time change in the sequence $\left(e_{i}\right)_{1 \leqslant i \leqslant n}$. More precisely, let $m$ be the number of time changes in $\gamma$. We define the function $\phi:\{1, \ldots, n-m\} \rightarrow \mathbb{N}$ by setting $\phi(1)=1$ and

$$
\forall i \in\{1, \ldots, n-m\} \quad \phi(i+1)=\left\{\begin{array}{lll}
\phi(i)+1 & \text { if } & e_{\phi(i)} \neq e_{\phi(i)+1} \\
\phi(i)+2 & \text { if } & e_{\phi(i)}=e_{\phi(i)+1}
\end{array} .\right.
$$

The sequence $\left(e_{\phi(i)}\right)_{1 \leqslant i \leqslant n-m}$ is called the space projection of $\gamma$, it is denoted by $\operatorname{Space}(\gamma)$. We say that length $(\operatorname{Space}(\gamma))$ is the length of the $\operatorname{STP} \gamma$, denoted also by length $(\gamma)$. We shall distinguish $\operatorname{Space}(\gamma)$ from the support of $\gamma$, denoted by $\operatorname{support}(\gamma)$, which we define as:

$$
\operatorname{support}(\gamma)=\operatorname{support}(\operatorname{Space}(\gamma)) \text {. }
$$


We say that a sequence of edges $\Gamma=\left(e_{i}\right)_{1 \leqslant i \leqslant k}$ is visitable if there exists a STP $\gamma$ such that $\operatorname{Space}(\gamma)=\Gamma$.

We prove next a key inequality to control the number of closing events along a simple STP.

Proposition 4.5. Let $\Gamma$ be a visitable sequence of edges and $[s, t]$ a time interval. For any $k \in$ $\{0, \ldots,|\operatorname{support}(\Gamma)|\}$ and any percolation configuration $y$ such that there are exactly $k$ closed edges in support $(\Gamma)$, we have the following inequality:

$$
P\left(\begin{array}{c}
\exists \gamma \text { decreasing closed } \\
\text { simple } S T P \text { in } Y \text { during }[s, t] \\
\text { such that } \operatorname{Space}(\gamma)=\Gamma
\end{array} \mid Y_{s}=y\right) \leqslant\left(\frac{(t-s)(1-p)}{|\Lambda|}\right)^{\operatorname{length}(\Gamma)-k} .
$$

Proof: We denote by $n$ the length of $\Gamma$ and $\left(e_{1}, \ldots, e_{n}\right)$ the sequence $\Gamma$. We consider a STP $\gamma$ such that $\operatorname{Space}(\gamma)=\Gamma$. Since $\gamma$ is closed, all the edges of $\Gamma$ are closed at time $s$ or become closed after $s$. For an edge $e \in \operatorname{support}(\Gamma)$, we denote by $v(e)$ the number of times that $\Gamma$ visits $e$ :

$$
v(e)=\left|\left\{j \in\{1, \ldots, n\}: e_{j}=e\right\}\right| .
$$

Since $\gamma$ is simple, between two consecutive visits, there exists a time when the edge $e$ is open, as illustrated in the Figure 4.5.

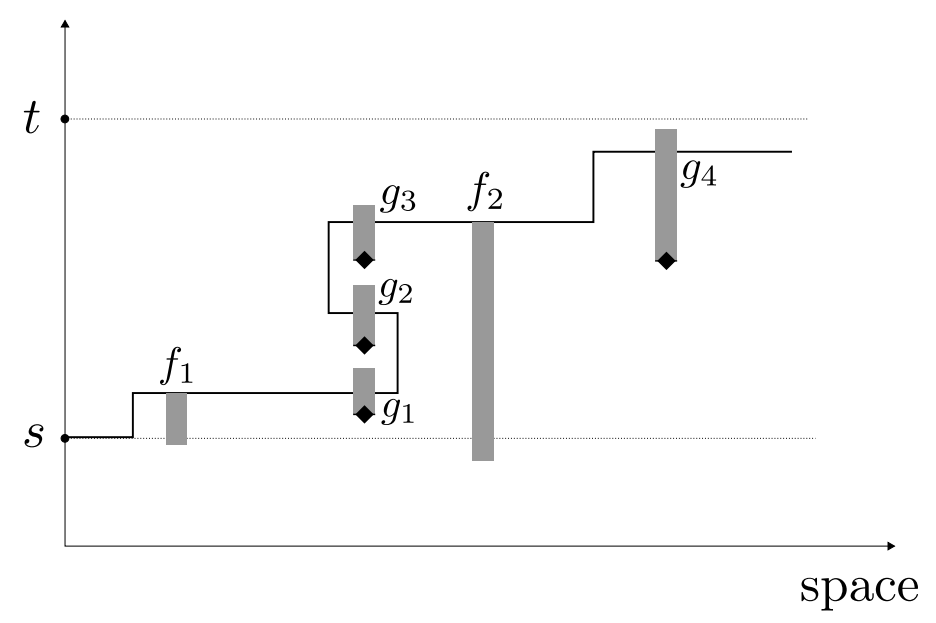

Figure 4.5. The edges $f_{1}, f_{2}$ are closed at time $s$. The edges $g_{1}, g_{2}, g_{3}$ and $g_{4}$ closes after $s$. We see that $g_{1}=g_{2}=g_{3}$ and the simplicity of the path implies that the edge opens and closes between two consecutive visits.

For each edge $e$ visited by $\gamma$, we distinguish two cases according to the configuration $Y_{s}$. If $Y_{s}(e)=1$, there is a time between $s$ and the first visit when $e$ becomes closed and the edge $e$ closes at least $v(e)$ times during the time interval $] s, t]$. If $Y_{s}(e)=0$, then, between the time $s$ and the first visit of $e$, it can remain closed and the edge $e$ becomes closed at least $v(e)-1$ times during $] s, t]$. Notice that the numbers $v(e)$ depend on the sequence $\Gamma$. The probability in the proposition is therefore less than or equal to

$$
\left.P\left(\left\{\begin{array}{c}
\{e \in \operatorname{support}(\Gamma) \quad y(e)=1 \\
e \text { closes at least } v(e) \text { times during }] s, t]
\end{array}\right\} \cap \begin{array}{c}
\forall f \in \operatorname{support}(\Gamma) \quad y(f)=0 \\
f \text { closes at least } v(f)-1 \text { times during }] s, t]
\end{array}\right\} \mid Y_{s}=y\right) .
$$

Notice that for any edge $e$ such that $y(e)=1$ (respectively $y(e)=0$ ), the event

$$
\{e \text { closes at least } v(e)(\text { resp. } v(e)-1) \text { times during }] s, t]\}
$$


depends on the collection of random variables

$$
F(e)=\left\{\left(E_{r}, B_{r}\right): s<r \leqslant t, E_{r}=e, B_{r}=0\right\} .
$$

Therefore these events are independent of the event $\left\{Y_{s}=y\right\}$ which depends on $\left(X_{0}, Y_{0}\right)$ and $\left\{\left(E_{r}, B_{r}\right): r \leqslant s\right\}$. The probability in (4.1) is thus equal to

$$
\left.P\left(\begin{array}{c}
\left\{\begin{array}{c}
\forall e \in \operatorname{support}(\Gamma) \quad y(e)=1 \\
e \text { closes at least } v(e) \text { times during }] s, t]
\end{array}\right\} \cap \\
\forall f \in \operatorname{support}(\Gamma) \quad y(f)=0 \\
f \text { closes at least } v(f)-1 \text { times during }] s, t]
\end{array}\right\}\right) .
$$

For any edge $e \in \operatorname{support}(\Gamma)$, we define $J(e)$ as the set of indices

$$
J(e)=\left\{s<j \leqslant t: E_{j}=e, B_{j}=0\right\} .
$$

We notice that the sets $(J(e), e \in \operatorname{support}(\Gamma))$ are pairwise disjoint subsets of $\mathbb{N}^{*}$. By the BK inequality applied to the random variables $\left(E_{t}, B_{t}\right)_{t \in \mathbb{N}}$, the probability in (4.1) is less than

$$
\begin{aligned}
& \left.\left.\prod_{e \in \operatorname{support}(\Gamma), y(e)=1} P(e \text { closes at least } v(e) \text { times during }] s, t\right]\right) \\
& \left.\left.\times \prod_{f \in \operatorname{support}(\Gamma), y(f)=0} P(f \text { closes at least } v(f)-1 \text { times during }] s, t\right]\right) .
\end{aligned}
$$

We obtain therefore

$$
\begin{gathered}
P\left(\begin{array}{c}
\exists \gamma \text { decreasing closed simple STP } \\
\text { in } Y \text { during }[s, t] \quad \operatorname{Space}(\gamma)=\Gamma
\end{array} \mid Y_{s}=y\right) \\
\left.\left.\leqslant \prod_{e \in \operatorname{support}(\Gamma), y(e)=1} P(e \text { closes } v(e) \text { times during }] s, t\right]\right) \\
\left.\left.\times \prod_{f \in \operatorname{support}(\Gamma), y(f)=0} P(f \text { closes } v(f)-1 \text { times during }] s, t\right]\right) .
\end{gathered}
$$

For any edge $e \in \operatorname{support}(\Gamma)$ and any $m \in \mathbb{N}$, we have

$P(e$ closes at least $m$ times during $] s, t])$

$$
\leqslant P\left(\begin{array}{ccc}
\exists J \subset\{s+1, \ldots, t\} & |J|=m \\
\forall j \in J \quad E_{j}=e & B_{j}=0
\end{array}\right) \leqslant\left(\frac{(t-s)(1-p)}{|\Lambda|}\right)^{m} .
$$

We use this inequality in (4.2) and we obtain

$$
\begin{aligned}
& P\left(\begin{array}{c|c}
\exists \gamma \text { decreasing closed simple STP } & Y_{s}=y \\
\text { in } Y \text { during }[s, t] & \operatorname{Space}(\gamma)=\Gamma
\end{array}\right) \\
& \leqslant\left(\frac{(t-s)(1-p)}{|\Lambda|}\right) \sum_{e \in \operatorname{support}(\Gamma), y(e)=1} v(e)+\sum_{f \in \operatorname{support}(\Gamma), y(f)=0} v(f)-1 \\
& =\left(\frac{(t-s)(1-p)}{|\Lambda|}\right)^{n-k} .
\end{aligned}
$$

This is the desired result. 
4.3. Proof of Proposition 4.1. Our goal is to study the speed of a cut during a time interval of size $|\Lambda|$. We start by using the results in the previous section to control the length of a STP far from a cut.

Proposition 4.6. Let $\ell$ be a positive constant, $\Gamma$ be a visitable sequence of edges starting from an edge $e$ such that $\Gamma$ travels a distance less than $\ell$ and $t$ be a time. For $s \in \mathbb{N}$, we have the following inequality:

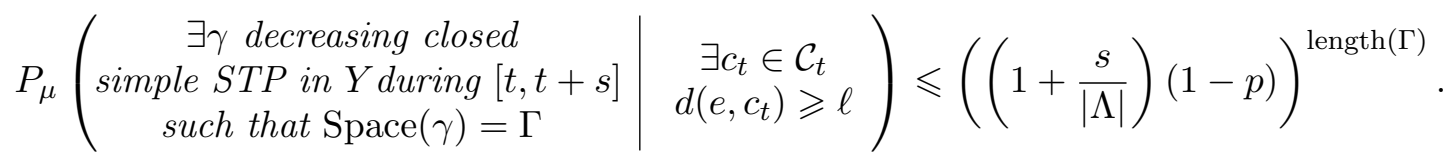

Proof: We start by rewriting the conditional probability in the proposition as

$$
\begin{aligned}
& P_{\mu}\left(\begin{array}{c}
\exists \gamma \text { decreasing closed } \\
\text { simple STP in } Y \text { during }[t, t+s] \\
\text { such that } \operatorname{Space}(\gamma)=\Gamma
\end{array} \mid \exists c_{t} \in \mathcal{C}_{t}, d\left(e, c_{t}\right) \geqslant \ell\right)= \\
& \frac{P_{\mu}\left(\left\{\begin{array}{c}
\exists \gamma \text { decreasing closed } \\
\text { simple STP in } Y \text { during }[t, t+s] \\
\text { such that Space }(\gamma)=\Gamma
\end{array}\right\} \cap\left\{\begin{array}{c}
\exists c_{t} \in \mathcal{C}_{t} \\
d\left(e, c_{t}\right) \geqslant \ell
\end{array}\right\}\right)}{P_{\mu}\left(\begin{array}{c}
\exists c_{t} \in \mathcal{C}_{t} \\
d\left(e, c_{t}\right) \geqslant \ell
\end{array}\right)}
\end{aligned}
$$

We denote by $\left(e_{i}, t_{i}\right)_{1 \leqslant i \leqslant N}$ the time-edges of $\gamma$. Let $n$ be the length of $\gamma$. We consider the case where there are exactly $k$ edges of $\operatorname{support}(\gamma)$ that are closed at time $t$. We shall estimate the following probability:

$$
P_{\mu}\left(\left\{\begin{array}{c}
\exists \gamma \text { simple decreasing STP } \\
\text { closed in } Y \text { of length } n \\
\gamma \text { starts at }(e, t+s) \text { and ends after } t \\
\operatorname{Space}(\gamma)=\Gamma \\
\exists F \subset \operatorname{support}(\gamma)|F|=k \\
\forall f \in F \quad Y_{t}(f)=0 \\
\forall f \in \operatorname{support}(\gamma) \backslash F \quad Y_{t}(f)=1 \\
\left\{\exists c_{t} \in \mathcal{C}_{t}, d\left(e, c_{t}\right) \geqslant \ell\right\}
\end{array}\right\} \cap\right) .
$$

We consider the set $M(k)$ of the configurations defined as

$$
M(k)=\left\{\begin{aligned}
& \exists F \subset \operatorname{support}(\Gamma),|F|=k \\
& \omega: \quad \forall f \in F \quad \omega(f)=0 \\
& \forall f \in \operatorname{support}(\Gamma) \backslash F \quad \omega(f)=1 \\
& \exists C \in \mathcal{C} \quad d(e, C) \geqslant \ell
\end{aligned}\right\} .
$$

The probability in (4.3) is bounded from above by

$$
\sum_{y \in M(k)} P_{\mu}\left(\begin{array}{c}
\exists \gamma \text { decreasing simple closed STP, } \\
\text { length }(\gamma)=n, \quad \text { Space }(\gamma)=\Gamma, \\
\gamma \text { starts at }(e, t+s) \text { and ends after } t
\end{array} \mid Y_{t}=y\right) P_{\mu}\left(Y_{t}=y\right)
$$

By Proposition 4.5, for any $y \in M(k)$, we have

$$
P_{\mu}\left(\begin{array}{c}
\exists \gamma \text { decreasing simple closed STP, } \\
\text { length }(\gamma)=n, \quad \text { Space }(\gamma)=\Gamma, \\
\gamma \text { starts at }(e, t+s) \text { and ends after } t
\end{array} \mid Y_{t}=y\right) \leqslant\left(\frac{s}{|\Lambda|}(1-p)\right)^{n-k} .
$$


We compute now the probability $P_{\mu}\left(Y_{t} \in M(k)\right)$. Notice that

$$
P_{\mu}\left(Y_{t} \in M(k)\right) \leqslant P_{\mu}\left(\begin{array}{cc}
\exists F & \subset \operatorname{support}(\Gamma) \quad|F|=k \\
\forall f \in F & Y_{t}(f)=0 \\
\exists c_{t} \in \mathcal{C}_{t} & d\left(e, c_{t}\right) \geqslant \ell
\end{array}\right) .
$$

The event in the last probability depends only on the configuration $Y_{t}$. Since the initial configuration $\left(X_{0}, Y_{0}\right)$ is distributed according to $\mu_{p}$, so is the couple $\left(X_{t}, Y_{t}\right)$. The configuration $Y_{t}$ is distributed according to the second marginal distribution $P_{\mathcal{D}}$. We have therefore

$$
P_{\mu}\left(\begin{array}{c}
\exists F \subset \operatorname{support}(\Gamma) \quad|F|=k \\
\forall f \in F \quad Y_{t}(f)=0 \\
\exists c_{t} \in \mathcal{C}_{t} \quad d\left(e, c_{t}\right) \geqslant \ell
\end{array}\right)=P_{\mathcal{D}}\left(\begin{array}{c}
\exists F \subset \operatorname{support}(\Gamma) \quad|F|=k \\
\forall f \in F \quad f \text { closed } \\
\exists C \in \mathcal{C} \quad d(e, C) \geqslant \ell
\end{array}\right) .
$$

By the definition of $P_{\mathcal{D}}$, we have

$$
\begin{aligned}
& P_{\mathcal{D}}\left(\begin{array}{c}
\exists F \subset \operatorname{support}(\Gamma) \quad|F|=k \\
\forall f \in F \quad f \text { closed } \\
\exists C \in \mathcal{C} \quad d(e, C) \geqslant \ell
\end{array}\right) \\
& =P_{p}\left(\begin{array}{c|c}
\exists F \subset \operatorname{support}(\Gamma) \quad|F|=k & \\
\forall f \in F \quad f \text { closed } & T \longleftrightarrow B \\
\exists C \in \mathcal{C} \quad d(e, C) \geqslant \ell &
\end{array}\right) \\
& =\frac{P_{p}\left(\left\{\begin{array}{c}
\exists F \subset \operatorname{support}(\Gamma) \quad|F|=k \\
\forall f \in F \quad f \text { closed }
\end{array}\right\} \cap\left\{\begin{array}{c}
\exists C \in \mathcal{C} \\
d(e, C) \geqslant \ell
\end{array}\right\} \cap\{T \longleftrightarrow B\}\right)}{P_{p}(T \longleftrightarrow B)} .
\end{aligned}
$$

The existence of a cut implies the event $\{T \longleftrightarrow B\}$, thus we can rewrite the numerator as

$$
P_{p}\left(\left\{\begin{array}{c}
\exists F \subset \operatorname{support}(\Gamma) \quad|F|=k \\
\forall f \in F \quad f \text { closed }
\end{array}\right\} \bigcap\{\exists C \in \mathcal{C}, d(e, C) \geqslant \ell\}\right) .
$$

The edges of support $(\Gamma)$ are at distance less than $\ell$ from the edge $e$ and the event $\{\exists C \in \mathcal{C}, d(e, C) \geqslant$ $\ell\}$ depends on the edges at distance more than $\ell$ from $e$. It follows that the two events in the previous probability are independent and we have

$$
\begin{aligned}
P_{p}\left(\left\{\begin{array}{c}
\exists F \subset \operatorname{support}(\Gamma) \quad|F| \\
\forall f \in F \quad f \text { closed }
\end{array}\right\} \bigcap\{\exists C \in \mathcal{C}, d(e, C) \geqslant \ell\}\right) \\
=P_{p}\left(\begin{array}{c}
\exists F \subset \operatorname{support}(\Gamma) \quad|F|=k \\
\forall f \in F \quad f \text { closed }
\end{array}\right) P_{p}(\exists C \in \mathcal{C}, d(e, C) \geqslant \ell) .
\end{aligned}
$$

Replacing the numerator in (4.5) by this product, we obtain

$$
\begin{gathered}
P_{p}\left(\left\{\begin{array}{r}
\exists F \subset \operatorname{support}(\Gamma) \quad|F|=k \\
\forall f \in F \quad f \text { closed }
\end{array}\right\} \cap\{\exists C \in \mathcal{C}, d(e, C) \geqslant \ell\} \mid T \leftrightarrow B\right) \\
=\frac{P_{p}\left(\begin{array}{c}
\exists F \subset \operatorname{support}(\Gamma) \quad|F|=k \\
\forall f \in F \quad f \text { closed }
\end{array}\right) P_{p}(\exists C \in \mathcal{C}, d(e, C) \geqslant \ell)}{P_{p}(T \leftrightarrow B)} \\
=P_{p}\left(\begin{array}{c}
\exists F \subset \operatorname{support}(\Gamma) \quad|F|=k \\
\forall f \in F \quad f \text { closed }
\end{array}\right) P_{\mathcal{D}}(\exists C \in \mathcal{C}, d(e, C) \geqslant \ell) .
\end{gathered}
$$

Since the edges of $F$ are distinct, we have

$$
P_{p}\left(\begin{array}{c}
\exists F \subset \operatorname{support}(\Gamma) \quad|F|=k \\
\forall f \in F \quad f \text { closed }
\end{array}\right) \leqslant\left(\begin{array}{c}
|\operatorname{support}(\Gamma)| \\
k
\end{array}\right)(1-p)^{k} .
$$


Combined with (4.4), we obtain that, for $\Gamma$ and $k$ fixed, the probability in (4.3) is bounded from above by

$$
\left(\begin{array}{c}
|\operatorname{support}(\Gamma)| \\
k
\end{array}\right)\left(\frac{s}{|\Lambda|}\right)^{n-k}(1-p)^{n} P_{\mathcal{D}}(\exists C \in \mathcal{C}, d(e, C) \geqslant \ell) .
$$

We sum on the number $k$ from 0 to $|\operatorname{support}(\Gamma)|$, and we recall that

$$
|\operatorname{support}(\Gamma)| \leqslant n \text {. }
$$

We have therefore

$$
\begin{gathered}
P_{\mu}\left(\left\{\begin{array}{c}
\exists \gamma \operatorname{simple} \text { closed decreasing STP, } \\
\text { length }(\gamma)=n, \quad \text { Space }(\gamma)=\Gamma, \\
\gamma \text { starts at }(e, t+s) \text { and ends after } t
\end{array}\right\} \cap\left\{\begin{array}{c}
\exists c_{t} \in \mathcal{C}_{t} \\
d\left(e, c_{t}\right) \geqslant \ell
\end{array}\right\}\right) \\
\leqslant \sum_{0 \leqslant k \leqslant \ell / 2 d}\left(\begin{array}{l}
n \\
k
\end{array}\right)\left(\frac{s}{|\Lambda|}\right)^{n-k}(1-p)^{n} P_{\mathcal{D}}(\exists C \in \mathcal{C}, d(e, C) \geqslant \ell) \\
=\left(\left(1+\frac{s}{|\Lambda|}\right)(1-p)\right)^{n} P_{\mathcal{D}}(\exists C \in \mathcal{C}, d(e, C) \geqslant \ell)
\end{gathered}
$$

Since the second marginal of $P_{\mu}$ is $P_{\mathcal{D}}$, we have

$$
P_{\mu}\left(\begin{array}{c}
\exists c_{t} \in \mathcal{C}_{t} \\
d\left(e, c_{t}\right) \geqslant \ell
\end{array}\right)=P_{\mathcal{D}}(\exists C \in \mathcal{C}, d(e, C) \geqslant \ell)
$$

This yields the inequality in the proposition.

We can now estimate the probability that the set of the pivotal edges moves fast. To do so, we study the STP constructed in Proposition 4.4 and we use the previous results.

Proof of Proposition 4.1: We rewrite the conditional probability appearing in the proposition as

$$
\frac{P_{\mu}\left(\left\{e \in \mathcal{P}_{t+s}\right\} \cap\left\{\forall r \in[t, t+s] \quad \mathcal{P}_{r} \neq \emptyset\right\} \cap\left\{\exists c_{t} \in \mathcal{C}_{t}, d\left(e, c_{t}\right) \geqslant \ell\right\}\right)}{P_{\mu}\left(\exists c_{t} \in \mathcal{C}_{t}, d\left(e, c_{t}\right) \geqslant \ell\right)} .
$$

Let us estimate the probability in the numerator. By Proposition 4.4, there exists a closed decreasing simple STP $\gamma$ inside of $\Lambda$ which connects $(e, t+s)$ to either an edge of $\mathcal{P}_{t}$ at time $t$ or to an edge intersecting the boundary of $\Lambda$ after time $t$. In both cases, this STP travels a distance at least $\ell$ because all the edges of $\mathcal{P}_{t}$ are included in the cuts and $e$ is at distance more than $\ell$ from $\Lambda^{c}$. Since the STP is a $*$-STP, the distance between two consecutive edges is at most $d$, and the length of the $\mathrm{STP}$ is at least $(\ell-1) / d$. We denote by $\left(e_{i}, t_{i}\right)_{1 \leqslant i \leqslant N}$ the time-edges of $\gamma$. Let $n$ be the first index such that the STP

is longer than $\ell / 2 d$, i.e.,

$$
\left(e_{1}, t_{1}\right), \ldots,\left(e_{n}, t_{n}\right)
$$

$$
n=\inf \left\{k \geqslant 1: \operatorname{length}\left(\left(e_{1}, t_{1}\right), \ldots,\left(e_{k}, t_{k}\right)\right) \geqslant \frac{\ell}{2 d}\right\} .
$$

We set $\Gamma=\operatorname{Space}\left(\left(e_{i}, t_{i}\right)_{1 \leqslant i \leqslant n}\right)$ and we denote $\Gamma=\left(f_{i}\right)_{i \in I}$. We have the following inequality:

$$
\begin{aligned}
& P_{\mu}\left(\begin{array}{c}
e \in \mathcal{P}_{t+s} \\
\forall r \in[t, t+s] \quad \mathcal{P}_{r} \neq \emptyset
\end{array} \mid \exists c_{t} \in \mathcal{C}_{t}, d\left(e, c_{t}\right) \geqslant \ell\right) \\
& \leqslant \sum_{\Gamma} P_{\mu}\left(\begin{array}{c|c}
\exists \gamma \operatorname{simple} \text { closed decreasing STP, } & \exists c_{t} \in \mathcal{C}_{t}, \\
\text { length }(\gamma)=\ell / 2 d, \quad \text { Space }(\gamma)=\Gamma, & d\left(e, c_{t}\right) \geqslant \ell \\
\gamma \operatorname{starts} \text { at }(e, t+s) \text { and ends after } t &
\end{array}\right)
\end{aligned}
$$


By Proposition 4.6, each term in the sum is less than

$$
\left(\left(1+\frac{s}{|\Lambda|}\right)(1-p)\right)^{\ell / 2 d}
$$

We sum next on all the possible choices of $\Gamma$. By Lemma 3.3, we have

$$
P_{\mu}\left(\begin{array}{c}
e \in \mathcal{P}_{t+s} \\
\forall r \in[t, t+s] \quad \mathcal{P}_{r} \neq \emptyset
\end{array} \mid \exists c_{t} \in \mathcal{C}_{t}, d\left(e, c_{t}\right) \geqslant \ell\right) \leqslant\left(\alpha(d)\left(1+\frac{s}{|\Lambda|}\right)(1-p)\right)^{\ell / 2 d} .
$$

There is a constant $\tilde{p}<1$ such that, for all $p \geqslant \tilde{p}, s \leqslant|\Lambda|$ and $\ell \geqslant 2$,

$$
\left(\alpha(d)\left(1+\frac{s}{|\Lambda|}\right)(1-p)\right)^{\ell / 2 d} \leqslant e^{-\ell} .
$$

We have obtained the result stated in the Proposition 4.1.

\section{The localisation around pivotal edges}

We start by stating a corollary of Proposition 4.1. Recall at first that the Hausdorff distance between two subsets $A$ and $B$ of $\mathbb{R}^{d}$, denoted by $d_{H}(A, B)$, is

$$
d_{H}(A, B)=\max \left\{\sup _{a \in A} d(a, B), \sup _{b \in B} d(b, A)\right\} .
$$

For $A$ a subset of $\mathbb{R}^{d}$ and $r>0$, we define the neighbourhood

$$
\mathcal{V}(A, r)=\left\{x \in \mathbb{R}^{d}: d(x, A)<r\right\} .
$$

The Hausdorff distance is also equal to

$$
\inf \{r \geqslant 0: A \subset \mathcal{V}(B, r), \quad B \subset \mathcal{V}(A, r)\} \text {. }
$$

For $\ell \geqslant 0$, we consider two subsets $A, B$ of $\Lambda$ and we define a semi-distance between two such subsets, denoted by $d_{H}^{\ell}(A, B)$, adapted to our study, by

$$
d_{H}^{\ell}(A, B)=\inf \left\{r \geqslant 0: \begin{array}{ll}
A \backslash \mathcal{V}\left(\Lambda^{c}, \ell\right) \subset \mathcal{V}(B, r) \\
B \backslash \mathcal{V}\left(\Lambda^{c}, \ell\right) \subset \mathcal{V}(A, r)
\end{array}\right\}
$$

Notice that $d_{H}^{\ell}$ is a semi-distance, in fact the triangle inequality is not satisfied. However, the following lemma allow us to compare $d_{H}^{\ell}$ with the Hausforff distance and provides us an alternative to the triangle inequality.

Lemma 5.1. For two subsets $A, B$ of $\Lambda$ and for all $\ell \geqslant 0$, we have

$$
d_{H}^{\ell}(A, B) \vee \ell \geqslant d_{H}\left(A \cup \Lambda^{c}, B \cup \Lambda^{c}\right) .
$$

Proof: Let $A, B$ be two subsets of $\Lambda$, and let us set $d_{1}=d_{H}^{\ell}(A, B)$. We claim that

$$
A \cup \Lambda^{c} \subset \mathcal{V}\left(B \cup \Lambda^{c}, d_{1} \vee \ell\right) \text {. }
$$

Let $x \in A \cup \Lambda^{c}$, we will show that $x$ belongs to $\mathcal{V}\left(B \cup \Lambda^{c}, d_{1} \vee \ell\right)$. We distinguish two cases. If $x \in \mathcal{V}\left(\Lambda^{c}, \ell\right)$, then we have

$$
x \in \mathcal{V}\left(B \cup \Lambda^{c}, \ell\right) \subset \mathcal{V}\left(B \cup \Lambda^{c}, d_{1} \vee \ell\right) .
$$

In the other case, if $x \in A \backslash \mathcal{V}\left(\Lambda^{c}, \ell\right)$ and by the definition of $d_{H}^{\ell}$, we have

$$
x \in \mathcal{V}\left(B, d_{1}\right) \subset \mathcal{V}\left(B \cup \Lambda^{c}, d_{1}\right) \subset \mathcal{V}\left(B \cup \Lambda^{c}, d_{1} \vee \ell\right)
$$

By exchanging $A$ and $B$, we have

$$
B \cup \Lambda^{c} \subset \mathcal{V}\left(A \cup \Lambda^{c}, d_{1} \vee \ell\right)
$$

By the definition of $d_{H}$, we obtain the desired claim, which in turn proves the lemma. 
Proposition 5.2. We have the following result:

$$
\begin{aligned}
\exists \tilde{p}<1 \quad \exists \kappa>1 \quad \forall p \geqslant \tilde{p} \quad \forall c \geqslant 1 \quad \forall \Lambda \quad|\Lambda| \geqslant(c d)^{c d^{2} \quad \forall t \geqslant 0} \\
P_{\mu}\left(\exists s \leqslant|\Lambda| \quad d_{H}^{\kappa c \ln |\Lambda|}\left(\mathcal{P}_{t}, \mathcal{P}_{t+s}\right) \geqslant \kappa c \ln |\Lambda|\right) \leqslant \frac{10 d}{|\Lambda|^{c}} .
\end{aligned}
$$

Proof: We fix $s \in\{1, \ldots,|\Lambda|\}$. By the definition of the distance $d_{H}^{\ell}$, we have, for any $\kappa>1$,

$$
\begin{aligned}
& P_{\mu}\left(d_{H}^{\kappa c \ln |\Lambda|}\left(\mathcal{P}_{t}, \mathcal{P}_{t+s}\right) \geqslant \kappa c \ln |\Lambda|\right) \leqslant \\
& P_{\mu}\left(\mathcal{P}_{t+s} \backslash \mathcal{V}\left(\Lambda^{c}, \kappa c \ln |\Lambda|\right) \not \mathcal{V}\left(\mathcal{P}_{t}, \kappa c \ln |\Lambda|\right)\right) \\
& +P_{\mu}\left(\mathcal{P}_{t} \backslash \mathcal{V}\left(\Lambda^{c}, \kappa c \ln |\Lambda|\right) \nsubseteq \mathcal{V}\left(\mathcal{P}_{t+s}, \kappa c \ln |\Lambda|\right)\right) .
\end{aligned}
$$

Since the two probabilities in the sum depend only on the process $Y$, which is reversible, they are in fact equal to each other. We shall estimate the first probability. We discuss first the case where there is a time $r \in\{t, \ldots, t+s\}$ when $\mathcal{P}_{r}=\emptyset$. By Proposition 3.5, there is a $\tilde{p}<1$ such that, for $p \geqslant \tilde{p}$ and all $\Lambda$,

$$
\forall r \in \mathbb{N} \quad P_{\mathcal{D}}\left(\mathcal{P}_{r}=\emptyset\right) \leqslant d|\Lambda| \exp (-D),
$$

where $D$ is the diameter of $T$. By summing over the time $r$, we have

$$
P_{\mathcal{D}}\left(\exists r \in[t, t+s] \quad \mathcal{P}_{r}=\emptyset\right) \leqslant d|\Lambda|^{2} \exp (-D) .
$$

We now consider the case where there exists always at least one pivotal edge during the time interval $[t, t+s]$. We can then apply Proposition 4.1 with an $\ell$ which will be determined later. There exists $\tilde{p}<1$ such that for $p \geqslant \tilde{p}$, for $t \geqslant 0$, and for any $s \leqslant|\Lambda|$ and $e$ an edge such that $d\left(e, \Lambda^{c}\right) \geqslant \ell$,

$$
P_{\mu}\left(\begin{array}{c}
e \in \mathcal{P}_{t+s} \\
\forall r \in[t, t+s] \quad \mathcal{P}_{r} \neq \emptyset
\end{array} \mid \exists c_{t} \in \mathcal{C}_{t}, d\left(e, c_{t}\right) \geqslant \ell\right) \leqslant e^{-\ell} .
$$

Let us fix $t \geqslant 0, s \leqslant|\Lambda|$ and $e$ an edge such that $d\left(e, \Lambda^{c}\right) \geqslant \ell$. The previous inequality implies that

$$
P_{\mu}\left(\begin{array}{c}
e \in \mathcal{P}_{t+s} \\
\forall r \in[t, t+s], \mathcal{P}_{r} \neq \emptyset \\
\exists c_{t} \in \mathcal{C}_{t} \quad d\left(e, c_{t}\right) \geqslant \ell
\end{array}\right) \leqslant e^{-\ell}
$$

In order to replace $c_{t}$ by $\mathcal{P}_{t}$ in the last probability, we use the Corollary 3.4. At the time $t$, the configuration $Y_{t}$ follows the distribution $P_{\mathcal{D}}$. Therefore, there exists $\tilde{p}<1$ and a $\kappa^{\prime}>1$ such that for $p \geqslant \tilde{p}$, for all $c \geqslant 1$ and all $\Lambda$ such that $|\Lambda| \geqslant 3^{6 d}$, we have

$$
P_{\mu}\left(\begin{array}{c}
\exists C \in \mathcal{C}_{t} \quad \exists f \in C \\
d\left(f, \Lambda^{c} \cup \mathcal{P}_{t} \backslash\{f\}\right) \geqslant \kappa^{\prime} c \ln |\Lambda| \\
\forall r \in[t, t+s] \quad \mathcal{P}_{r} \neq \emptyset
\end{array}\right) \leqslant \frac{1}{|\Lambda|^{c}} .
$$

From now onwards, we suppose that $p$ is larger than the three previous $\tilde{p}$. Let $c>0$ be fixed and let $\kappa^{\prime}$ be associated to $c$ as above. We distinguish two cases to control the following probability:

$$
\begin{gathered}
P_{\mu}\left(e \in \mathcal{P}_{t+s}, d\left(e, \mathcal{P}_{t}\right) \geqslant \kappa c \ln |\Lambda|, \forall r \in[t, t+s] \mathcal{P}_{r} \neq \emptyset\right) \leqslant \\
\qquad P_{\mu}\left(\begin{array}{c}
e \in \mathcal{P}_{t+s}, \quad d\left(e, \mathcal{P}_{t}\right) \geqslant \kappa c \ln |\Lambda|, \\
\forall C \in \mathcal{C}_{t} \quad \forall f \in C \backslash \mathcal{V}\left(\Lambda^{c}, \kappa^{\prime} c \ln |\Lambda|\right) \\
d\left(f, \mathcal{P}_{t}\right)<\kappa^{\prime} c \ln |\Lambda|, \\
\forall r \in[t, t+s] \quad \mathcal{P}_{r} \neq \emptyset \\
+P_{\mu}\left(\exists C \in \mathcal{C}_{t}, \exists f \in C, d\left(f, \Lambda^{c} \cup \mathcal{P}_{t} \backslash\{f\}\right) \geqslant \kappa^{\prime} c \ln |\Lambda|\right) .
\end{array}\right.
\end{gathered}
$$

The second probability is less than $1 /|\Lambda|^{c}$. Let us study the first probability. Since all the edges of a cut at time $t$ are either at distance less than $\kappa^{\prime} c \ln |\Lambda|$ from $\Lambda^{c}$ or at distance less than $\kappa^{\prime} c \ln |\Lambda|$ 
from $\mathcal{P}_{t}$ and the distance between $e$ and $\mathcal{P}_{t} \cup \Lambda^{c}$ is larger than $\kappa c \ln |\Lambda|$, then all the cuts at time $t$ are at distance more than $\left(\kappa-\kappa^{\prime}\right) c \ln |\Lambda|$ from $e$. Hence, for $\kappa>\kappa^{\prime}$,

$$
\begin{gathered}
P_{\mu}\left(\begin{array}{c}
e \in \mathcal{P}_{t+s} \quad d\left(e, \Lambda^{c} \cup \mathcal{P}_{t}\right) \geqslant \kappa c \ln |\Lambda| \\
\forall C \in \mathcal{C}_{t} \quad \forall f \in C \backslash \mathcal{V}\left(\Lambda^{c}, \kappa^{\prime} c \ln |\Lambda|\right) \\
d\left(f, \mathcal{P}_{t}\right)<\kappa^{\prime} c \ln |\Lambda| \\
\forall r \in[t, t+s] \quad \mathcal{P}_{r} \neq \emptyset
\end{array}\right) \leqslant \\
P_{\mu}\left(\begin{array}{c}
e \in \mathcal{P}_{t+s} d\left(e, \Lambda^{c}\right) \geqslant \kappa c \ln |\Lambda| \\
\forall C \in \mathcal{C}_{t} \quad \forall f \in C \backslash \mathcal{V}\left(\Lambda^{c}, \kappa^{\prime} c \ln |\Lambda|\right) \\
d(f, e)>\left(\kappa-\kappa^{\prime}\right) c \ln |\Lambda| \\
\forall r \in[t, t+s] \quad \mathcal{P}_{r} \neq \emptyset
\end{array}\right) \leqslant \\
P_{\mu}\left(\begin{array}{r}
\exists c_{t} \in \mathcal{C}_{t} \quad d\left(e, c_{t}\right) \geqslant\left(\kappa-\kappa^{\prime}\right) c \ln |\Lambda| \\
\forall r \in[t, t+s]
\end{array}\right) \leqslant \frac{1}{|\Lambda|_{r}^{\left(\kappa-\kappa^{\prime}\right) c}} . \emptyset
\end{gathered}
$$

We choose now $\kappa=\kappa^{\prime}+1$, and we get

$$
P_{\mu}\left(e \in \mathcal{P}_{t+s}, d\left(e, \mathcal{P}_{t}\right) \geqslant \kappa c \ln |\Lambda|, \forall r \in[t, t+s] \mathcal{P}_{r} \neq \emptyset\right) \leqslant \frac{2}{|\Lambda|^{c}} .
$$

We sum over $e$ in $\Lambda$ and $s \in\{1, \ldots,|\Lambda|\}$ to get

$$
P_{\mu}\left(\begin{array}{c}
\exists s \leqslant|\Lambda|, \exists e \in \mathcal{P}_{t+s} \\
d\left(e, \Lambda^{c} \cup \mathcal{P}_{t}\right) \geqslant \kappa c \ln |\Lambda| \\
\forall r \in[t, t+s] \quad \mathcal{P}_{r} \neq \emptyset
\end{array}\right) \leqslant \frac{4 d}{|\Lambda|^{c-2}}
$$

We add the probability in (5.2) and we obtain

$$
P_{\mu}\left(\begin{array}{c}
\exists s \leqslant|\Lambda|, \exists e \in \mathcal{P}_{t+s} \\
d\left(e, \Lambda^{c} \cup \mathcal{P}_{t}\right) \geqslant \kappa c \ln |\Lambda|
\end{array}\right) \leqslant \frac{4 d}{|\Lambda|^{c-2}}+d|\Lambda|^{2} \exp (-D) .
$$

This is the first probability in (5.1) and we conclude that

$$
P_{\mu}\left(\begin{array}{c}
\exists s \leqslant|\Lambda| \\
d_{H}^{\Lambda}\left(\mathcal{P}_{t}, \mathcal{P}_{t+s}\right) \geqslant \kappa c \ln |\Lambda|
\end{array}\right) \leqslant \frac{8 d}{|\Lambda|^{c-2}}+2 d|\Lambda|^{2} \exp (-D) .
$$

For all box $\Lambda$ such that $|\Lambda| \geqslant(c d)^{c d^{2}}$, we have

$$
\exp (-D) \leqslant \frac{1}{|\Lambda|^{c}}
$$

Therefore, for all $\Lambda$ such that $|\Lambda| \geqslant(c d)^{c d^{2}}$, we have

$$
\frac{8 d}{|\Lambda|^{c-2}}+2 d|\Lambda|^{2} \exp (-D) \leqslant \frac{10 d}{|\Lambda|^{c-2}} .
$$

In order to obtain $1 /|\Lambda|^{c}$, we replace $c$ by $c+2$, since $(c+2) / c \leqslant 3$ for $c \geqslant 1$, we have, for $|\Lambda| \geqslant \max \left\{(c d)^{c d^{2}}, 3^{6 d}\right\}$

$$
P_{\mu}\left(\begin{array}{c}
\exists s \leqslant|\Lambda| \\
d_{H}^{\Lambda}\left(\mathcal{P}_{t}, \mathcal{P}_{t+s}\right) \geqslant 3 \kappa c \ln |\Lambda|
\end{array}\right) \leqslant \quad P_{\mu}\left(\begin{array}{c}
\exists s \leqslant|\Lambda| \\
d_{H}^{\Lambda}\left(\mathcal{P}_{t}, \mathcal{P}_{t+s}\right) \geqslant \kappa(c+2) \ln |\Lambda|
\end{array}\right) \leqslant \frac{10 d}{|\Lambda|^{c}}
$$

This yields the desired inequality.

We now complete the proof of the Theorem 1.1. 
Proof of Theorem 1.1: Let us fix an edge $e$ in $\Lambda$ and a time $t$. We distinguish the cases where $e \in \mathcal{I}_{t} \backslash \mathcal{P}_{t}$ and $e \in \mathcal{P}_{t}$. If $e \in \mathcal{P}_{t}$, then we use the Proposition 1.4. We consider now the case where $e \in \mathcal{I}_{t} \backslash \mathcal{P}_{t}$. We consider the last time $\tau$ when $e$ was pivotal,

$$
\tau=\max \left\{0 \leqslant s<t: e \in \mathcal{P}_{s}, e \notin \mathcal{P}_{s+1}\right\} .
$$

The edge $e$ has not been modified between $\tau$ and $t$. Let $c \geqslant 1$. We have

$$
P_{\mu}(t-\tau \geqslant c|\Lambda| \ln |\Lambda|) \leqslant P_{\mu}\left(\begin{array}{c}
\forall r \in[t-c|\Lambda| \ln |\Lambda|, t] \\
E_{r} \neq e
\end{array}\right) \leqslant \frac{1}{|\Lambda|^{c}} .
$$

We consider now the case where $t-\tau<c|\Lambda| \ln |\Lambda|$. We split the interval $[\tau, t]$ into subintervals of length $|\Lambda|$ and we set

$$
t_{i}=\tau+i|\Lambda|, \quad 0 \leqslant i<\frac{t-\tau}{|\Lambda|} \quad \text { and } \quad t_{\lfloor(t-\tau) /|\Lambda|\rfloor+1}=t .
$$

According to Proposition 5.2, there exists $\tilde{p}<1$ and $\kappa^{\prime}>1$, such that

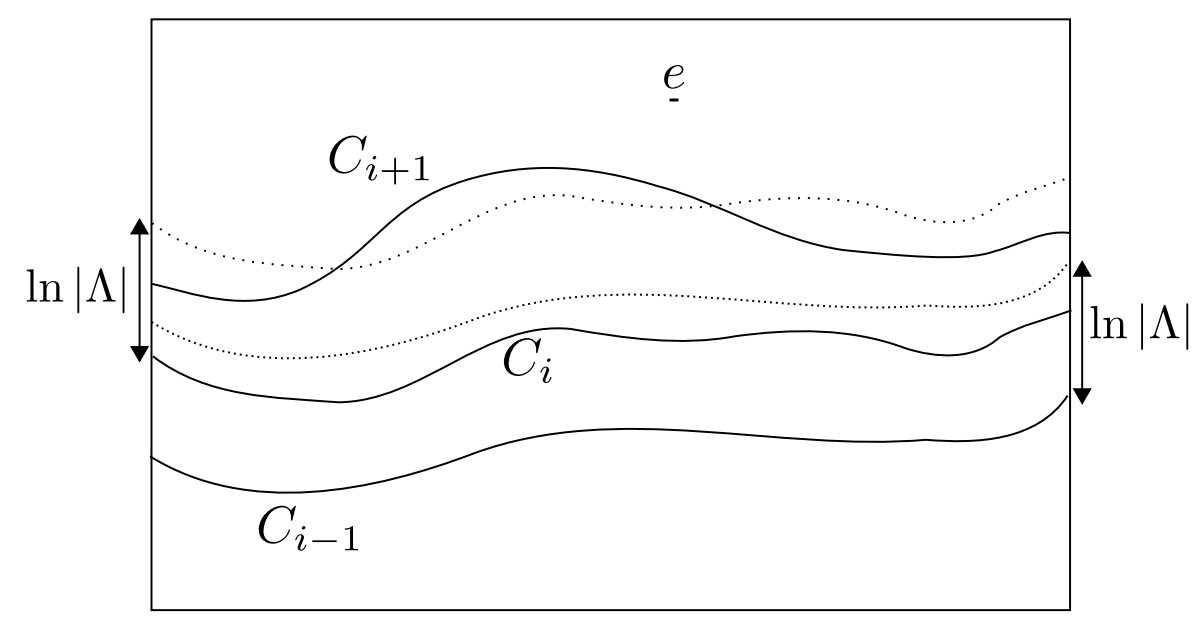

Figure 5.6. The cut $C_{i}$ at time $t_{i}$ is at distance less than $\ln |\Lambda|$ from $C_{i-1}$ and the cut $C_{i+1}$ is at distance more than $\ln |\Lambda|$ from $C_{i}$.

$$
\forall p \geqslant \tilde{p} \quad \forall c \geqslant 1 \quad \forall|\Lambda| \geqslant(c d)^{c d^{2}} \forall j \geqslant 0 \quad P_{\mu}\left(d_{H}^{\kappa^{\prime} c \ln |\Lambda|}\left(\mathcal{P}_{t_{j}}, \mathcal{P}_{t_{j+1}}\right) \geqslant \kappa^{\prime} c \ln |\Lambda|\right) \leqslant \frac{10 d}{|\Lambda|^{c}}
$$

Let $c \geqslant 1$. We suppose that

$$
d\left(e, \mathcal{P}_{t} \cup \Lambda^{c}\right) \geqslant 2 \kappa^{\prime} c^{2}(\ln |\Lambda|)^{2}>(c \ln |\Lambda|+1) \kappa^{\prime} c \ln |\Lambda| .
$$

We have by Lemma 5.1, as illustrated in the Figure 5.6,

$$
\begin{aligned}
& \sum_{0 \leqslant i<(t-\tau) /|\Lambda|} d_{H}^{\kappa^{\prime} c \ln |\Lambda|}\left(\mathcal{P}_{t_{i}}, \mathcal{P}_{t_{i+1}}\right) \vee \kappa^{\prime} c \ln |\Lambda| \\
& \geqslant \sum_{\substack{0 \leqslant i<(t-\tau) /|\Lambda|\\
}} d_{H}\left(\mathcal{P}_{t_{i}} \cup \Lambda^{c}, \mathcal{P}_{t_{i+1}} \cup \Lambda^{c}\right) \\
& \left.\quad \mathcal{P}_{\tau} \cup \Lambda^{c}, \mathcal{P}_{t} \cup \Lambda^{c}\right) \geqslant d\left(e, \mathcal{P}_{t} \cup \Lambda^{c}\right) \geqslant 2 \kappa^{\prime} c^{2}(\ln |\Lambda|)^{2} .
\end{aligned}
$$

Necessarily, there is an index $0 \leqslant j<c \ln |\Lambda|$ such that

$$
d_{H}^{\kappa^{\prime} c \ln |\Lambda|}\left(\mathcal{P}_{t_{j}}, \mathcal{P}_{t_{j+1}}\right) \vee \kappa^{\prime} c \ln |\Lambda| \geqslant 2 \kappa^{\prime} c \ln |\Lambda| .
$$


Therefore, we have

$$
d_{H}^{\kappa^{\prime} c \ln |\Lambda|}\left(\mathcal{P}_{t_{j}}, \mathcal{P}_{t_{j+1}}\right) \geqslant \kappa^{\prime} c \ln |\Lambda| .
$$

By summing over $j$ from 0 to $\lfloor c \ln |\Lambda|\rfloor$, we have

$$
P_{\mu}\left(e \in \mathcal{I}_{t}, d\left(e, \mathcal{P}_{t}\right) \geqslant 2 \kappa^{\prime} c^{2}(\ln |\Lambda|)^{2}, t-\tau<c|\Lambda| \ln |\Lambda|\right) \leqslant \frac{10 d(c \ln |\Lambda|+1)}{|\Lambda|^{c}}
$$

We set $\kappa=2 \kappa^{\prime}$ and we obtain

$$
\begin{aligned}
& P_{\mu}\left(e \in \mathcal{P}_{t} \cup \mathcal{I}_{t}, d\left(e, \mathcal{P}_{t} \backslash\{e\}\right) \geqslant \kappa c^{2}(\ln |\Lambda|)^{2}\right) \\
& \qquad P_{\mu}\left(e \in \mathcal{P}_{t}, d\left(e, \mathcal{P}_{t} \backslash\{e\}\right) \geqslant \kappa(c \ln |\Lambda|)^{2}\right) \\
& +P_{\mu}(t-\tau \geqslant c|\Lambda| \ln |\Lambda|)+P_{\mu}\left(e \in \mathcal{I}_{t}, d\left(e, \mathcal{P}_{t}\right) \geqslant \kappa(c \ln |\Lambda|)^{2}, t-\tau \leqslant c \ln |\Lambda|\right) \\
& \leqslant \frac{2}{|\Lambda|^{c}}+\frac{10 d(c \ln |\Lambda|+1)}{|\Lambda|^{c}} .
\end{aligned}
$$

We sum over the edge $e$. For $\Lambda$ such that $|\Lambda| \geqslant(c d)^{c d^{2}}$, we have

$$
P_{\mu}\left(\exists e \in \mathcal{P}_{t} \cup \mathcal{I}_{t}, d\left(e, \mathcal{P}_{t} \backslash\{e\}\right) \geqslant \kappa(c \ln |\Lambda|)^{2}\right) \leqslant \frac{4 d+20 d^{2}(c \ln |\Lambda|+1)}{|\Lambda|^{c-1}} \leqslant \frac{1}{|\Lambda|^{c-2}} .
$$

We apply this result with $c+2$ instead of $c$, since for $c \geqslant 1,(c+2)^{2} / c^{2} \leqslant 9$, we have

$$
9 \kappa c^{2} \geqslant \kappa(c+2)^{2} \text {. }
$$

Therefore, we have

$$
P_{\mu}\left(\exists e \in \mathcal{P}_{t} \cup \mathcal{I}_{t}, d\left(e, \mathcal{P}_{t} \backslash\{e\}\right) \geqslant 9 \kappa(c \ln |\Lambda|)^{2}\right) \leqslant \frac{1}{|\Lambda|^{c}} .
$$

This proves the statement of Theorem 1.1.

\section{Speed estimations conditioned by the past}

We derive further estimates on the speed of the pivotal edges which will be used in the proof of the Theorem 1.3. First, we give a corollary of the Proposition 4.1, which provides a control on the cuts, rather than the pivotal edges.

Corollary 6.1. We have the following inequality:

$$
\begin{aligned}
\exists \tilde{p}<1 \quad \forall p \geqslant \tilde{p} \quad \forall \Lambda & \\
& \forall \ell \geqslant 1 \quad \forall e \in \Lambda \quad d\left(e, \Lambda^{c}\right) \geqslant \ell \quad \forall t>0 \quad \forall s \in\{0, \ldots,|\Lambda|\} \\
& P_{\mu}\left(\exists C \in \mathcal{C}_{t+s}, e \in C \mid \exists c_{t} \in \mathcal{C}_{t}, d\left(e, c_{t}\right) \geqslant \ell\right) \leqslant \exp (-\ell) .
\end{aligned}
$$

Proof: We adapt the construction of the STP done in the Proposition 4.4. We cannot use directly the STP constructed in Proposition 4.4 because between the times $t$ and $t+s$, the set of the pivotal edges can be empty. Therefore, we consider $\tau$ the last time before $t+s$ when $\mathcal{P}$ is empty, i.e.,

$$
\tau=\sup \left\{r \leqslant t+s: \mathcal{P}_{r}=\emptyset\right\} \text {. }
$$

If $\tau \leqslant t$, the conditions of Proposition 4.4 are satisfied and there exists a closed decreasing simple STP starting from $(e, t+s)$ and ending after $t$ which travels a distance at least $\ell$. If $\tau=t+s$, since the edge $e$ is in a cut, there exists a closed $*$-path in $Y_{t+s}$ which connects $e$ to an edge intersecting the boundary of $\Lambda$. This path travels a distance at least $\ell$. If $t<\tau<t+s$, then we have $\mathcal{P}_{r} \neq \emptyset$ for $\tau<r \leqslant t+s$. According to Proposition 4.4, there exists a STP from $(e, t+s)$ to an edge of $\mathcal{P}_{\tau+1}$ at time $\tau+1$ or an edge intersecting the boundary of $\Lambda$ after time $\tau+1$. If the STP ends 
at an edge intersecting the boundary, then it travels a distance at least $\ell$. If it ends at an edge of $\mathcal{P}_{\tau+1}$ at time $\tau+1$, then, at time $\tau+1$, there must be an edge which becomes open and creates the pivotal edges of $\mathcal{P}_{\tau+1}$ which are on a cut $C$ at time $\tau+1$. Notice that the cut $C$ existed already at time $\tau$ because all the edges of $C$ are closed. Therefore, there exists a decreasing closed STP which connects $(e, t+s)$ to an edge intersecting the boundary of $\Lambda$ at time $\tau$. We reapply the algorithm of modification described in the proof of Proposition 4.4 to obtain a simple STP. In all the cases above, we obtain a decreasing closed simple STP starting at $(e, t+s)$ which travels a distance at least $\ell$. We apply the same arguments as in the proof of Proposition 4.1 in order to obtain the desired estimate.

We wish to control the movement of the set of the cuts over a time interval. To achieve this goal, we will derive estimates for the appearance of a pivotal edge conditionally on the presence of a cut far away during a whole interval. In Proposition 4.1, the conditioning gave information on one instant, not a whole interval. In the next lemma, we deal with a time interval of length $|\Lambda|$.

Lemma 6.2. There exist $\tilde{p}<1$ and $\kappa>0$ such that for $p \geqslant \tilde{p}$, any $c \geqslant 1$, any integer $m \geqslant 1$, any $\Lambda$ such that $|\Lambda| \geqslant 2 d$, any edge $e$ at distance more than $\kappa c \ln |\Lambda|$ from $\Lambda^{c}$ and for $0<s \leqslant|\Lambda| \leqslant t$, we have

$$
P_{\mu}\left(\begin{array}{c|c}
\exists C \in \mathcal{C}_{t+s} & \forall r \in] t-|\Lambda|, t] \\
d(e, C) \leqslant(m-1) \kappa c \ln |\Lambda| & \begin{array}{c}
\exists C_{r} \in \mathcal{C}_{r} \quad d\left(e, C_{r}\right) \geqslant m \kappa c \ln |\Lambda| \\
\exists C^{\prime} \in \mathcal{C}_{t-|\Lambda|}
\end{array} \quad d\left(e, C^{\prime}\right) \geqslant(m+1) \kappa c \ln |\Lambda|
\end{array}\right) \leqslant \frac{1}{|\Lambda|^{c}}
$$

Proof: Let $\kappa$ be a positive constant which will be chosen at the end of the proof. We reuse the construction of the STP in Corollary 6.1: there exists a decreasing closed simple STP which connects $(e, t+s)$ to a pivotal edge at time $t$ or to an edge intersecting the boundary of $\Lambda$ at a time after $t$. Since the edge $e$ is at distance at least $\kappa c \ln |\Lambda|$ from $\mathcal{P}_{t} \cup \Lambda^{c}$, in both cases, there exists a decreasing closed simple STP $\gamma$ of length $(\kappa c \ln |\Lambda|) / 2 d$ starting from the time-edge $(e, t+s)$ and ending after $t$ which is strictly included in the box $\Lambda$. Let $\Gamma$ be the space projection of $\gamma$, i.e.,

$$
\Gamma=\operatorname{Space}(\gamma)=\left(e_{1}, \ldots, e_{m}\right) .
$$

We introduce the following events:

$$
\begin{gathered}
\left.\left.D_{1}=\{\forall r \in] t-|\Lambda|, t\right], \exists C_{r} \in \mathcal{C}_{r}, d\left(e, C_{r}\right) \geqslant m \kappa c \ln |\Lambda|\right\}, \\
\bar{D}_{1}=\left\{\exists C \in \mathcal{C}_{t-|\Lambda|}, d(e, C) \geqslant(m+1) \kappa c \ln |\Lambda|\right\},
\end{gathered}
$$

and

$$
\mathcal{E}(t, s, \Gamma)=\left\{\begin{array}{c}
\exists \gamma \text { simple closed decreasing STP } \\
\text { length }(\gamma)=(\kappa c \ln |\Lambda|) / 2 d, \operatorname{Space}(\gamma)=\Gamma \\
\gamma \text { starts at an edge }\left(e^{\prime}, t+s\right) \\
d\left(e^{\prime}, e\right) \leqslant(m-1) \kappa c \ln |\Lambda| \\
\text { and ends after } t
\end{array}\right\} .
$$

As in the proof of Proposition 4.1, the probability appearing in the proposition is less than

$$
\sum_{\Gamma} P_{\mu}\left(\mathcal{E}(t, s, \Gamma) \mid D_{1}, \bar{D}_{1}\right)
$$

where the sum is over the possible choices for $\Gamma$. We fix a path $\Gamma$ and we condition each probability in the sum by the configuration at time $t$. Let $A$ be a subset of $\operatorname{support}(\Gamma)$, we denote by $M(A)$ the following set of configurations:

$$
M(A)=\left\{\begin{array}{ll}
\omega: \quad \forall f \in A \quad \omega(f)=0 \\
& \forall f \in \operatorname{support}(\Gamma) \backslash A \quad \omega(f)=1
\end{array}\right\} .
$$

Let $y$ be a configuration in $M(A)$ and let us start by estimating the probability

$$
P_{\mu}\left(\mathcal{E}(t, s, \Gamma) \mid Y_{t}=y, D_{1}, \bar{D}_{1}\right) .
$$


By the Markov property, this probability is equal to

$$
P_{\mu}\left(\mathcal{E}(t, s, \Gamma) \mid Y_{t}=y\right)
$$

and by Proposition 4.5 , it is less than

$$
\left(\frac{s}{|\Lambda|}(1-p)\right)^{(\kappa c \ln |\Lambda|) / 2 d-|A|}
$$

Each term of the sum in (6.1) can be written as

$$
\sum_{0 \leqslant k \leqslant|\operatorname{support}(\Gamma)|} \sum_{\substack{A \subset \operatorname{support}(\Gamma) \\|A|=k}} \sum_{y \in M(A)} P_{\mu}\left(\mathcal{E}(t, s, \Gamma) \mid Y_{t}=y, D_{1}, \bar{D}_{1}\right) \times P_{\mu}\left(Y_{t}=y \mid D_{1}, \bar{D}_{1}\right) .
$$

Using (6.2), we see that each term in (6.1) is less than

$$
\sum_{0 \leqslant k \leqslant|\operatorname{support}(\Gamma)|}\left(\frac{s}{|\Lambda|}(1-p)\right)^{(\kappa c \ln |\Lambda|) / 2 d-k} \sum_{\substack{A \subset \operatorname{support}(\Gamma) \\|A|=k}} P_{\mu}\left(Y_{t} \in M(A) \mid D_{1}, \bar{D}_{1}\right) .
$$

In the rest of the proof, we will calculate an upper bound of

$$
\sum_{\substack{A \subset \operatorname{support}(\Gamma) \\|A|=k}} P_{\mu}\left(Y_{t} \in M(A) \mid D_{1}, \bar{D}_{1}\right) .
$$

Notice that, for an edge $f \in \Gamma$, if there is a time $r \in[t-|\Lambda|, t]$ such that $E_{r}=f$, then, under the probability $P_{p}$, conditioned on $D_{1}, \overline{D_{1}}$, the state of $f$ at time $t$ is independent of the other edges of $\Gamma$ and it follows a Bernoulli variable of parameter $p$. On the contrary, if $E_{r} \neq f$ for all $r \in[t-|\Lambda|, t]$, then the state of $f$ at time $t$ is the same as at time $t-|\Lambda|$. For $A$ a subset of $\operatorname{support}(\Gamma)$ and $B$ a subset of $A$, we define the event $\operatorname{reset}(B, A)$ as

$$
\operatorname{reset}(B, A)=\left\{\begin{array}{c}
\forall e \in B, \exists r \in[t-|\Lambda|, t], E_{r}=e \\
\forall r \in[t-|\Lambda|, t], E_{r} \notin A \backslash B
\end{array}\right\}
$$

For each subset $A$, we partition the probability in (6.4) according to the subset $B$ of $A$ for which the event $\operatorname{reset}(B, A)$ occurs, and we get

$$
\begin{aligned}
& \sum_{\substack{A \subset \operatorname{support}(\Gamma) \\
|A|=k, B \subset A}} P_{\mu}\left(Y_{t} \in M(A), \operatorname{reset}(B, A) \mid D_{1}, \bar{D}_{1}\right) \\
& \underset{\substack{A \subset \operatorname{support}(\Gamma) \\
|A|=k, B \subset A}}{=P_{\mu}}\left(\begin{array}{c|c}
\forall f \in B, Y_{t}(f)=0 \\
\forall f \in A \backslash B, Y_{t-|\Lambda|}(f)=0
\end{array} \mid D_{1}, \bar{D}_{1}\right) .
\end{aligned}
$$

We write

$$
P_{\mu}(\cdot)=\sum_{x_{0}, y_{0}} P_{x_{0}, y_{0}}(\cdot) \mu\left(\left(x_{0}, y_{0}\right)\right)
$$


where $P_{x_{0}, y_{0}}$ is the law of the process $\left(X_{t}, Y_{t}\right)_{t \in \mathbb{N}}$ starting from the initial configuration $\left(x_{0}, y_{0}\right)$. For each term we rewrite the conditioned probability as follows:

$$
\begin{aligned}
P_{x_{0}, y_{0}}\left(\begin{array}{c}
\forall f \in B, Y_{t}(f)=0 \\
\forall f \in A \backslash B, Y_{t-|\Lambda|}(f)=0 \\
\operatorname{reset}(B, A)
\end{array}\right. & \left.D_{1}, \bar{D}_{1}\right) \\
& \left.=\frac{P_{x_{0}, y_{0}}\left(\left\{\begin{array}{c}
\forall f \in B, Y_{t}(f)=0 \\
\forall f \in A \backslash B, Y_{t-|\Lambda|}(f)=0 \\
\operatorname{reset}(B, A)
\end{array}\right.\right.}{P_{x_{0}, y_{0}}\left(D_{1}, \bar{D}_{1}\right)} . \cap D_{1} \cap \bar{D}_{1}\right)
\end{aligned} .
$$

Starting from an initial configuration $\left(x_{0}, y_{0}\right)$, the process $\left(Y_{t}\right)_{t \in \mathbb{N}}$ is obtained by conditioning to stay in the configurations with disconnexion. We can replace $P_{\mu}$ by $P_{p}$ in the previous fraction and the numerator can be written as

$$
\begin{aligned}
P_{p}\left(\left\{\begin{array}{c}
\forall f \in B, X_{t}(f)=0 \\
\forall f \in A \backslash B, X_{t-|\Lambda|}(f)=0 \\
\operatorname{reset}(B, A)
\end{array}\right\} \bigcap D_{1} \bigcap \bar{D}_{1}\right) \\
\quad=P_{p}\left(\left\{\begin{array}{c}
\forall f \in B, B_{\tau(f)}=0 \\
\forall f \in A \backslash B, X_{t-|\Lambda|}(f)=0 \\
\operatorname{reset}(B, A)
\end{array}\right\} \bigcap D_{1} \bigcap \bar{D}_{1}\right),
\end{aligned}
$$

where the time $\tau(f)$ is the last time before $t$ when the edge $f$ is chosen, i.e.,

$$
\tau(f)=\sup \left\{s \leqslant t: E_{s}=f\right\}
$$

Let us fix a sequence of edges $\mathbf{e}=\left(e_{1}, \ldots, e_{t}\right)$ and let us condition this last probability by the event

$$
\left(E_{1}, \ldots, E_{t}\right)=\mathbf{e}
$$

We have

$$
\begin{aligned}
& P_{p}\left(\left\{\begin{array}{c}
\forall f \in B, B_{\tau(f)}=0 \\
\forall f \in A \backslash B, X_{t-|\Lambda|}(f)=0 \\
\operatorname{reset}(B, A)
\end{array}\right\} \bigcap D_{1} \bigcap \bar{D}_{1}\right) \\
&=\sum_{\mathbf{e} \in \operatorname{reset}(B, A)} P_{p}\left(\left\{\begin{array}{c}
\forall f \in B, B_{\tau(f)}=0 \\
\forall f \in A \backslash B, X_{t-|\Lambda|}(f)=0
\end{array}\right\} \cap D_{1} \cap \bar{D}_{1} \mid\right.\left.\left(E_{1}, \ldots, E_{t}\right)=\mathbf{e}\right) \\
& \times P_{p}\left(\left(E_{1}, \ldots, E_{t}\right)=\mathbf{e}\right) .
\end{aligned}
$$

Notice that on the event $\operatorname{reset}(B, A)$, for an edge $f \in B$, we have necessarily $\tau(f)>t-|\Lambda|$. Therefore the event $\left\{\forall f \in B, B_{\tau(f)}=0\right\}$ depends on the set of variables $\left\{B_{s}: e_{s} \in B, s>t-|\Lambda|\right\}$. The events

$$
\left\{\forall f \in A \backslash B, X_{t-|\Lambda|}(f)=0\right\}
$$

and $\bar{D}_{1}$ depend on the variables $\left\{B_{s}: s \leqslant t-|\Lambda|\right\}$ and the event $D_{1}$ does not depend on the variables $\left\{B_{s}: E_{s} \in B\right\}$. All the events above are decreasing, by the BK inequality applied to the 
random variables $\left(B_{s}\right)_{s \in \mathbb{N}}$, we have

$$
\begin{gathered}
P_{p}\left(\left\{\begin{array}{c}
\forall f \in B, B_{\tau(f)}=0 \\
\forall f \in A \backslash B, X_{t-|\Lambda|}(f)=0
\end{array}\right\} \cap D_{1} \cap \bar{D}_{1} \mid\left(E_{1}, \ldots, E_{t}\right)=\mathbf{e}\right) \\
\leqslant P_{p}\left(\forall f \in B, B_{\tau(f)}=0 \mid\left(E_{1}, \ldots, E_{t}\right)=\mathbf{e}\right) \\
\times P_{p}\left(\begin{array}{c}
\forall f \in A \backslash B, X_{t-|\Lambda|}(f)=0 \\
D_{1} \cap \bar{D}_{1}
\end{array}\right. \\
\leqslant(1-p)^{|B|} P_{p}\left(\begin{array}{c}
\forall f \in A \backslash B, X_{t-|\Lambda|}(f)=0 \\
D_{1} \cap \bar{D}_{1}
\end{array} \mid\left(E_{1}, \ldots, E_{t}\right)=\mathbf{e}\right) .
\end{gathered}
$$

We use this inequality in (6.7) and we obtain

$$
\begin{aligned}
P_{p}\left(\left\{\begin{array}{c}
\forall f \in B, B_{\tau(f)}=0 \\
\forall f \in A \backslash B, X_{t-|\Lambda|}(f) \\
\operatorname{reset}(B, A)
\end{array}\right\} \bigcap D_{1} \bigcap \bar{D}_{1}\right) \\
\leqslant(1-p)^{|B|} P_{p}\left(\left\{\begin{array}{c}
\forall f \in A \backslash B, X_{t-|\Lambda|}(f)=0 \\
\operatorname{reset}(B, A)
\end{array}\right\} \bigcap D_{1} \bigcap \bar{D}_{1}\right) .
\end{aligned}
$$

We replace the numerator in (6.6) and we sum over the initial configurations, we have

$$
\begin{aligned}
& P_{\mu}\left(\begin{array}{c|c}
\forall f \in B, Y_{t}(f)=0 & \\
\forall f \in A \backslash B, Y_{t-|\Lambda|}(f)=0 & D_{1}, \bar{D}_{1} \\
\operatorname{reset}(B, A) &
\end{array}\right) \\
& \leqslant(1-p)^{|B|} P_{\mu}\left(\begin{array}{c|c}
\forall f \in A \backslash B, X_{t-|\Lambda|}(f)=0 & D_{1}, \bar{D}_{1} \\
\operatorname{reset}(B, A) &
\end{array} .\right.
\end{aligned}
$$

This last probability is less than

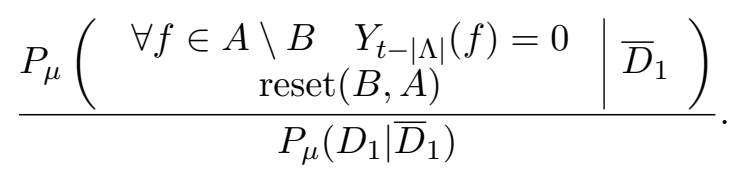

Let us estimate separately the numerator and the denominator. In order to calculate the numerator, we use the notation $\bar{M}(A)$ defined as follows:

$$
\bar{M}(A)=\{\omega: \forall f \in A \quad \omega(f)=0\} .
$$

We obtain

$$
P_{\mu}\left(\begin{array}{c}
\forall f \in A \backslash B \quad Y_{t-|\Lambda|}(f)=0 \\
\operatorname{reset}(B, A)
\end{array} \mid \bar{D}_{1}\right)=\sum_{y \in \bar{M}(A \backslash B)} P_{\mu}\left(\operatorname{reset}(B, A), Y_{t-|\Lambda|}=y \mid \bar{D}_{1}\right) .
$$

As in the proof of Proposition 4.1, we write

$$
P_{\mu}\left(\operatorname{reset}(B, A), Y_{t-|\Lambda|}=y \mid \bar{D}_{1}\right)=P_{\mu}\left(\operatorname{reset}(B, A) \mid Y_{t-|\Lambda|}=y, \bar{D}_{1}\right) P_{\mu}\left(Y_{t-|\Lambda|}=y \mid \bar{D}_{1}\right) .
$$

Since the event $\operatorname{reset}(B, A)$ depends only on the variables

$$
\left\{\left(E_{r}, B_{r}\right): t-|\Lambda|<r \leqslant t\right\},
$$

it is independent from $Y_{t-|\Lambda|}$ (and also from the event $\bar{D}_{1}$, as $\bar{D}_{1}$ is entirely determined by $Y_{t-|\Lambda|}$ ). We obtain

$$
\sum_{y \in \bar{M}(A \backslash B)} P_{\mu}\left(\operatorname{reset}(B, A), Y_{t-|\Lambda|}=y \mid \bar{D}_{1}\right)=P_{\mu}(\operatorname{reset}(B, A)) P_{\mu}\left(Y_{t-|\Lambda|} \in \bar{M}(A \backslash B) \mid \bar{D}_{1}\right) .
$$


Let us estimate the last probability. Since the second marginal of $P_{\mu}$ is $P_{\mathcal{D}}$ and $P_{\mathcal{D}}(\cdot)=P_{p}(\cdot \mid T \longleftrightarrow$ $B)$, we have

$$
P_{\mu}\left(Y_{t-|\Lambda|} \in \bar{M}(A \backslash B) \mid \bar{D}_{1}\right)=\frac{P_{p}\left(\begin{array}{c}
\forall f \in A \backslash B \quad f \text { closed } \\
\exists C \in \mathcal{C}, d(e, C) \geqslant(m+1) \kappa c \ln |\Lambda|
\end{array}\right)}{P_{\mathcal{D}}(\exists C \in \mathcal{C}, d(e, C) \geqslant(m+1) \kappa c \ln |\Lambda|) P_{p}(T \longleftrightarrow B)} .
$$

The event

$$
\{\forall f \in A \backslash B \quad f \text { closed }\}
$$

depends only on the edges at distance less than $(m-1 / 2) \kappa c \ln |\Lambda|$ from the edge $e$, while the event

$$
\{\exists C \in \mathcal{C}, d(e, C) \geqslant(m+1) \kappa c \ln |\Lambda|\}
$$

depends on the edges at distance larger than $(m+1) \kappa c \ln |\Lambda|$ from $e$. By independence, we have

$$
\begin{aligned}
& P_{p}\left(\begin{array}{c}
\forall f \in A \backslash B \quad f \text { closed } \\
\exists C \in \mathcal{C}, d(e, C) \geqslant(m+1) \kappa c \ln |\Lambda|
\end{array}\right)= \\
& \quad P_{p}(\forall f \in A \backslash B \quad f \text { closed }) P_{p}(\exists C \in \mathcal{C}, d(e, C) \geqslant(m+1) \kappa c \ln |\Lambda|) .
\end{aligned}
$$

We obtain therefore

$$
P_{\mu}\left(Y_{t-|\Lambda|} \in \bar{M}(A \backslash B) \mid \bar{D}_{1}\right) \leqslant P_{p}(\forall f \in A \backslash B \quad f \text { closed })=(1-p)^{|A \backslash B|} .
$$

We conclude that the numerator of (6.8) is less than

$$
(1-p)^{|A \backslash B|} P_{\mu}(\operatorname{reset}(B, A)) \text {. }
$$

Now, we estimate the denominator in (6.8). In fact, this probability is equal to

$$
\left.\left.1-P_{\mu}(\exists s \in] t-|\Lambda|, t\right], \forall C \in \mathcal{C}_{s}, d(e, C)<m \kappa c \ln |\Lambda| \mid \bar{D}_{1}\right) .
$$

By Corollary 6.1, there exists a $\tilde{p}<1$ such that for $p \geqslant \tilde{p}$, for any $c, \kappa_{1} \geqslant 1$ and for any edge $e$ at distance more than $\kappa_{1} c \ln |\Lambda|$ from $\Lambda^{c}$, we have

$$
P_{\mu}\left(\exists C \in \mathcal{C}_{t+s}, e \in C\left|\exists c_{t} \in \mathcal{C}_{t}, d\left(e, c_{t}\right) \geqslant \kappa_{1} c \ln \right| \Lambda \mid\right) \leqslant \frac{1}{|\Lambda|^{\kappa_{1} c}} .
$$

Since $(c+3) / c \leqslant 4$ for $c \geqslant 1$, there exists a $\kappa_{1}>1$, such that for any $c \geqslant 1$,

$$
\frac{1}{|\Lambda|^{\kappa_{1} c}} \leqslant \frac{1}{|\Lambda|^{c+3}}
$$

We have therefore, as illustrated in the Figure 6.7, for $|\Lambda| \geqslant 2 d$ :

$$
\begin{aligned}
& P_{\mu}\left(\begin{array}{c|c}
\exists s \in] t-|\Lambda|, t], \forall C^{\prime} \in \mathcal{C}_{s} & \bar{D}_{1} \\
d\left(e, C^{\prime}\right)<\kappa_{1} c \ln |\Lambda| &
\end{array}\right) \leqslant \\
& \sum_{s \in] t-|\Lambda|, t]} \sum_{f: d(e, f)<\kappa_{1} c \ln |\Lambda|} P_{\mu}\left(\exists C_{s} \in \mathcal{C}_{s}, f \in C_{s} \mid \bar{D}_{1}\right) \leqslant \\
& \sum_{s \in] t-|\Lambda|, t]} \sum_{f: d(e, f)<\kappa_{1} c \ln |\Lambda|} \frac{1}{|\Lambda|^{c+3}} \leqslant \frac{1}{|\Lambda|^{c}} \leqslant \frac{1}{2} .
\end{aligned}
$$

Let $\kappa \geqslant \kappa_{1}$, the probability (6.8) is less than

$$
2(1-p)^{|A \backslash B|} P_{\mu}(\operatorname{reset}(B, A)) .
$$




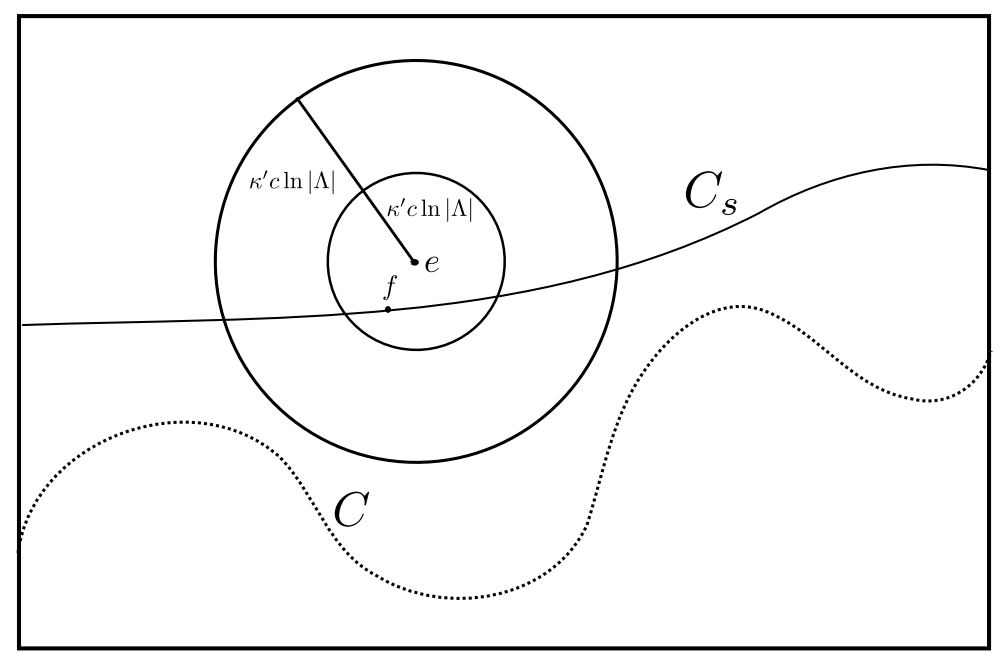

Figure 6.7. The edge $f \in \mathcal{P}_{s}$ is at distance less than $\kappa^{\prime} c \ln |\Lambda|$ from $e$ and the cut $C \in \mathcal{C}_{t-|\Lambda|}$ is at distance larger than $2 \kappa^{\prime} c \ln |\Lambda|$ from $e$.

We bound from above each term of (6.5) and we obtain an upper bound for (6.4):

$$
\sum_{\substack{\subset \operatorname{support}(\Gamma) \\|A|=k}} P_{\mu}\left(Y_{t} \in M(A) \mid D_{1}, \bar{D}_{1}\right) \leqslant \sum_{\substack{A \subset \operatorname{support}(\Gamma) \\|A|=k, B \subset A}} 2(1-p)^{k} P_{\mu}(\operatorname{reset}(B, A)) .
$$

For each set $A$ fixed, we have

$$
\sum_{B \subset A} P_{\mu}(\operatorname{reset}(B, A))=1
$$

Therefore, we obtain

$$
\begin{gathered}
P_{\mu}\left(Y_{t} \in M(A) \mid D_{1}, \bar{D}_{1}\right) \leqslant 2(1-p)^{|A|} \\
\sum_{\substack{A \subset \operatorname{support}(\Gamma) \\
|A|=k}} P_{\mu}\left(Y_{t} \in M(A) \mid D_{1}, \bar{D}_{1}\right) \leqslant 2\left(\begin{array}{c}
|\operatorname{support}(\Gamma)| \\
k
\end{array}\right)(1-p)^{k} .
\end{gathered}
$$

Finally, combined with (6.3), we obtain an upper bound for (6.1) which is

$$
\begin{aligned}
2(1-p)^{(\kappa c \ln |\Lambda|) / 2 d} \sum_{0 \leqslant k \leqslant(\kappa c \ln |\Lambda|) / 2 d}\left(\begin{array}{c}
|\operatorname{support}(\Gamma)| \\
k
\end{array}\right)\left(\frac{s}{|\Lambda|}\right)^{(\kappa c \ln |\Lambda|) / 2 d-k} & \\
& \leqslant 2\left((1-p)\left(1+\frac{s}{|\Lambda|}\right)\right)^{(\kappa c \ln |\Lambda|) / 2 d} .
\end{aligned}
$$

We sum over the possible choices for the path $\Gamma$, by the Lemma 3.3, the sum in (6.1) is less than

$$
2|\Lambda|(\alpha(d)(1-p)(1+s /|\Lambda|))^{(\kappa c \ln |\Lambda|) / 2 d} .
$$

There is a $\kappa>0$, such that for $p \geqslant \tilde{p}$, such that this term is less than

$$
\frac{1}{|\Lambda|^{c}} \text {. }
$$

We obtain the result stated in the lemma.

We next show a generalisation of Proposition 4.1 and Corollary 6.1 which is an essential ingredient for the proof of Theorem 1.3. 
Proposition 6.3. We have the following estimate:

$$
\begin{aligned}
& \exists \tilde{p}<1 \quad \exists \kappa>1 \quad \forall p \geqslant \tilde{p} \quad \forall c \geqslant 2 \quad \forall \Lambda \quad|\Lambda| \geqslant 12(2 \kappa d)^{d} \\
& \forall e \subset \Lambda \quad d\left(e, \Lambda^{c}\right) \geqslant \kappa c^{2} \ln ^{2}|\Lambda| \\
& \forall n \geqslant 1 \quad n \leqslant c \ln |\Lambda| \quad \forall m \geqslant 0 \quad n+m \leqslant c \ln |\Lambda| \\
& \forall s \in\{1, \ldots,|\Lambda|\} \quad \forall t \geqslant n|\Lambda| \\
& P_{\mu}\left(\begin{array}{c|c}
\forall k \in\{1, \ldots, n\} \\
\exists C \in \mathcal{C}_{t+s} & \forall r \in] t-k|\Lambda|, t-(k-1)|\Lambda|] \\
d(e, C) \leqslant m \kappa c \ln |\Lambda| & \begin{array}{c}
\exists C_{r} \in \mathcal{C}_{r} \quad d\left(e, C_{r}\right) \geqslant(k+m) \kappa c \ln |\Lambda| \\
\exists C^{\prime} \in \mathcal{C}_{t-n|\Lambda|}
\end{array} \quad d\left(e, C^{\prime}\right) \geqslant(n+m+1) \kappa c \ln |\Lambda|
\end{array}\right) \leqslant \frac{1}{|\Lambda|^{c}} .
\end{aligned}
$$

Proof: Notice that for the case $n=1$, this proposition corresponds to Lemma 6.2. Let $\kappa$ be a constant which will be determined at the end of the proof. We start by introducing some notations. For $m \in \mathbb{N}$ and $k \geqslant 1$, we define $D_{k, m}$ to be the event

$$
D_{k, m}=\left\{\begin{array}{c}
\forall r \in] t-k|\Lambda|, t-(k-1)|\Lambda|] \\
\exists C_{r} \in \mathcal{C}_{r} \quad d\left(e, C_{r}\right) \geqslant(k+m) \kappa c \ln |\Lambda|
\end{array}\right\}
$$

and $\bar{D}_{k, m}$ the event

$$
\bar{D}_{k, m}=\left\{\exists C \in \mathcal{C}_{t-k|\Lambda|} \quad d(e, C) \geqslant(k+m+1) \kappa c \ln |\Lambda|\right\} .
$$

For $\kappa \geqslant 1, c \geqslant 2, k \in \mathbb{N}^{*}, e \subset \Lambda$ and $t, s \in \mathbb{N}^{*}$, we denote by $\left(H_{k, m}\right)$ the following inequality:

$$
\left(H_{k, m}\right): \quad P_{\mu}\left(\begin{array}{c}
\exists C \in \mathcal{C}_{t+s} \\
d(e, C) \leqslant m \kappa c \ln |\Lambda|
\end{array} \mid D_{1, m}, \ldots, D_{k, m}, \bar{D}_{k, m}\right) \leqslant \frac{1}{|\Lambda|^{c}} .
$$

Our goal is to show that there exist $\tilde{p}<1$ and $\kappa>1$ such that for $p \geqslant \tilde{p}, c \geqslant 2, e \subset \Lambda$ at distance larger than $\kappa c^{2} \ln ^{2}|\Lambda|$ from $\Lambda^{c}, s \in\{1, \ldots,|\Lambda|\}$, the inequality $\left(H_{k, m}\right)$ holds for any $1 \leqslant k+m \leqslant c \ln |\Lambda|$ and $t \geqslant(k+m)|\Lambda|$. In particular, the inequality stated in the proposition corresponds to the case $(k, m)=(n, 0)$. In order to show this proposition by induction on $k$, we introduce an auxiliary inequality $\left(G_{k, m}\right)$ for $A \subset \Lambda, d(e, A) \leqslant(\kappa c \ln |\Lambda|) / 2$ :

$$
\left(G_{k, m}\right): P_{\mu}\left(\forall f \in A \quad Y_{t}(f)=0 \mid D_{1, m}, \ldots, D_{k+m}, \bar{D}_{k+m}\right) \leqslant 2^{k}(1-p)^{|A|} .
$$

By Lemma 6.2, there exist $\tilde{p}<1$ and $\kappa>1$ such that for $p \geqslant \tilde{p}, c \geqslant 2, e \subset \Lambda$ at distance larger than $\kappa c^{2} \ln ^{2}|\Lambda|$ from $\Lambda^{c}$ and $t \geqslant c|\Lambda| \ln |\Lambda|, s \in\{1, \ldots,|\Lambda|\}$, the inequalities $\left(H_{1, m}\right)$ hold for all $m \leqslant c \ln |\Lambda|-1$, meanwhile, the inequalities $\left(G_{1, m}\right)$ was also proved in (6.9). For this $\tilde{p}$, there exists a $\kappa>0$ such that, for any $c \geqslant 2$, we have

$$
\left(\alpha(d) 2^{1+2 d / \kappa}(1-\tilde{p})\right)^{(\kappa c \ln |\Lambda|) / 2 d} \leqslant \frac{1}{|\Lambda|^{c}} .
$$

Notice that for this $\kappa$, the inequality in Lemma 6.2 is also satisfied. Let us fix $c \geqslant 2$ and let us show the inequalities by induction on the integer $k$. Let $k<c \ln |\Lambda|$, we suppose that the inequalities $\left(H_{k, m}\right)$ and $\left(G_{k, m}\right)$ hold for all $m \in \mathbb{N}$ such that $k+m \leqslant c \ln |\Lambda|$. Let us prove first the inequality $\left(G_{k+1, m}\right)$ for a $m \in\{0, \ldots,\lfloor c \ln |\Lambda|\rfloor-k-1\}$. We reuse the notations $\operatorname{reset}(I, A, B)$ and $\bar{M}(A)$ defined for a subset $B$ of $A$ and a time interval $I$ :

$$
\begin{aligned}
\operatorname{reset}(I, A, B) & =\left\{\begin{array}{c}
\forall e \in B, \exists r \in I, E_{r}=e \\
\forall r \in I, E_{r} \notin A \backslash B
\end{array}\right\}, \\
\bar{M}(A) & =\{\omega: \forall f \in A \quad \omega(f)=0\} .
\end{aligned}
$$


We denote by $I_{1}$ the interval $\left.] t-|\Lambda|, t\right]$. We rewrite the probability $\left(G_{k+1, m}\right)$ as in the proof of Lemma 6.2:

$$
\begin{aligned}
P_{\mu}\left(\forall f \in A \quad Y_{t}(f)\right. & \left.=0 \mid D_{1, m}, \ldots, D_{k+1, m}, \bar{D}_{k+1, m}\right) \\
= & \sum_{B \subset A} P_{\mu}\left(\forall f \in A \quad Y_{t}(f)=0, \operatorname{reset}\left(I_{1}, A, B\right) \mid D_{1, m}, \ldots, D_{k+1, m}, \bar{D}_{k+1, m}\right) .
\end{aligned}
$$

For each $B \subset A$, we have

$$
\begin{aligned}
& P_{\mu}\left(\begin{array}{ll}
\forall f \in A \quad Y_{t}(f)=0, \operatorname{reset}\left(I_{1}, A, B\right) \mid D_{1, m}, \ldots, D_{k+1, m}, \bar{D}_{k+1, m}
\end{array}\right) \\
& =P_{\mu}\left(\begin{array}{c|c}
\forall f \in B, Y_{t}(f)=0 \\
\forall f \in \begin{array}{c}
A \backslash B, Y_{t-|\Lambda|}(f)=0 \\
\operatorname{reset}\left(I_{1}, A, B\right)
\end{array}
\end{array} \mid D_{1, m}, \ldots, D_{k+1, m}, \bar{D}_{k+1, m}\right) .
\end{aligned}
$$

We use the same arguments as in the inequality (6.6) of the Lemma 6.2 to obtain a factor $1-p$ for each edge where the event reset is realised. We have

$$
\begin{aligned}
& P_{\mu}\left(\begin{array}{c|c}
\forall f \in B, Y_{t}(f)=0 \\
\forall f \in A \backslash B, Y_{t-|\Lambda|}(f)=0 \\
\operatorname{reset}\left(I_{1}, A, B\right)
\end{array}\right. \\
& \quad \leqslant(1-p)^{|B|} P_{\mu}\left(Y_{t-|\Lambda|} \in \bar{M}(A \backslash B), \operatorname{reset}\left(I_{1}, A, B\right) \mid D_{1, m}, \ldots, D_{k+1, m}, \bar{D}_{k+1, m}\right)
\end{aligned}
$$

The event $\operatorname{reset}\left(I_{1}, A, B\right)$ is independent of what happens before and until $t-|\Lambda|$ and of $D_{2, m}, \ldots, D_{k+1, m}, \bar{D}_{k+1, m}$. Therefore, this last probability is less than or equal to

$$
\begin{gathered}
\frac{P_{\mu}\left(Y_{t-|\Lambda|} \in \bar{M}(A \backslash B), \operatorname{reset}\left(I_{1}, A, B\right) \mid D_{2, m}, \ldots, D_{k+1, m} \bar{D}_{k+1, m}\right)}{P_{\mu}\left(D_{1, m} \mid D_{2, m}, \ldots, D_{k+1, m}, \bar{D}_{k+1, m}\right)}= \\
\frac{P_{\mu}\left(\operatorname{reset}\left(I_{1}, A, B\right)\right)}{P_{\mu}\left(D_{1, m} \mid D_{2, m}, \ldots, D_{k+1, m}, \bar{D}_{k+1, m}\right)} \\
\times P_{\mu}\left(Y_{t-|\Lambda|} \in \bar{M}(A \backslash B) \mid D_{2, m}, \ldots, D_{k+1, m}, \bar{D}_{k+1, m}\right) .
\end{gathered}
$$

We apply the inequality $\left(G_{k, m+1}\right)$, at time $t-|\Lambda|$. The last probability is less or equal than

$$
2^{k}(1-p)^{|A \backslash B|} \text {. }
$$

For the denominator, we apply $\left(H_{k, m+1}\right)$ at time $t-1$ and we obtain

$$
\begin{aligned}
P_{\mu}\left(D_{1, m} \mid D_{2, m}, \ldots, D_{k+1, m}, \bar{D}_{k+1, m}\right) & \\
& \geqslant 1-P_{\mu}\left(\begin{array}{c}
\exists r \in] t-|\Lambda|, t] \quad \exists C_{r} \in \mathcal{C}_{r} \\
d\left(e, C_{r}\right) \leqslant m \kappa c \ln |\Lambda|
\end{array} D_{2, m}, \ldots, D_{k+1, m}, \bar{D}_{k+1, m}\right) \geqslant 1-\frac{|\Lambda|}{|\Lambda|^{c}} .
\end{aligned}
$$

Therefore, for $|\Lambda| \geqslant 2$, we have

$$
\frac{1}{|\Lambda|^{c-1}} \leqslant \frac{1}{2}
$$

Therefore, we have for the denominator

$$
P_{\mu}\left(D_{1, m} \mid D_{2, m}, \ldots, D_{k+1, m}, \bar{D}_{k+1, m}\right) \geqslant \frac{1}{2} .
$$


We obtain $\left(G_{k+1, m}\right)$ by summing over the choices of $B$ :

$$
\begin{aligned}
P_{\mu}\left(\forall f \in A \quad Y_{t}(f)=0 \mid D_{1, m}, \ldots, D_{k+1, m}, \bar{D}_{k+1, m}\right) & \\
& \leqslant \frac{2^{k}(1-p)^{|A|}}{1 / 2} \sum_{B \subset A} P_{\mu}\left(\operatorname{reset}\left(I_{1}, A, B\right)\right)=2^{k+1}(1-p)^{|A|} .
\end{aligned}
$$

In order to obtain $\left(H_{k+1, m}\right)$, we will study the STP obtained as in the Corollary 6.1. We recall that this STP is of length at least $(\kappa c \ln |\Lambda|) / 2 d$. We fix first the space projection of the STP, which we denote by $\Gamma$. As in the proof of Lemma 6.2 and Proposition 4.1, we study separately the edges that close after the time $t$ and the edges which are closed at time $t$ by conditioning the probability by the configuration $Y_{t}$. For the edges which become closed after $t$, we apply Proposition 4.5 and we obtain that the probability for obtaining a simple closed decreasing STP $\gamma$ between $t$ and $t+s$ satisfying $\operatorname{Space}(\gamma)=\Gamma$ is less than

$$
\begin{aligned}
\sum_{0 \leqslant j \leqslant \operatorname{support}(\Gamma)} \sum_{A \subset \operatorname{support}(\Gamma):|A|=j}\left(\frac{s}{|\Lambda|}(1-p)\right)^{(\kappa c \ln |\Lambda|) / 2 d-j} \times \\
P\left(Y_{t} \in \bar{M}(A) \mid D_{1, m}, \ldots, D_{k+1, m}, \bar{D}_{k+1, m}\right) .
\end{aligned}
$$

We apply the inequality $\left(G_{k+1, m}\right)$ for the last probability and we have

$$
P\left(Y_{t} \in \bar{M}(A) \mid D_{1, m}, \ldots, D_{k+1, m}, \bar{D}_{k+1, m}\right) \leqslant 2^{k+1}(1-p)^{j} .
$$

Therefore, the sum in (6.10) is less than

$$
\begin{aligned}
& \sum_{0 \leqslant j \leqslant \operatorname{support}(\Gamma)}\left(\begin{array}{c}
\operatorname{support}(\Gamma) \\
j
\end{array}\right)\left(\frac{s}{|\Lambda|}\right)^{j} 2^{k+1}(1-p)^{(\kappa c \ln |\Lambda|) / 2 d} \leqslant \\
& 2^{k+1}((1+s /|\Lambda|)(1-p))^{(\kappa c \ln |\Lambda|) / 2 d} .
\end{aligned}
$$

For $|\Lambda| \geqslant 2 d, k+1 \leqslant c \ln |\Lambda|$ and $s \leqslant|\Lambda|$, we have

$$
2^{k+1}((1+s /|\Lambda|)(1-p))^{(\kappa c \ln |\Lambda|) / 2 d} \leqslant\left(2^{1+2 d / \kappa}(1-p)\right)^{(\kappa c \ln |\Lambda|) / 2 d} .
$$

We sum over the choices for $\Gamma$ by using the Lemma 3.3, and we have

$$
\begin{aligned}
P_{\mu}\left(\begin{array}{c}
\exists C \in \mathcal{C}_{t+s} \\
d(e, C) \leqslant m \kappa c \ln |\Lambda|
\end{array} \mid D_{1, m}, \ldots, D_{k+1, m}, \bar{D}_{k+1, m}\right) & \\
& \leqslant|\Lambda|\left(\alpha(d) 2^{1+2 d / \kappa}(1-p)\right)^{(\kappa c \ln |\Lambda|) / 2 d} .
\end{aligned}
$$

For $p \geqslant \tilde{p}$ and the $\kappa$ chosen at the beginning of the proof, for any $c \geqslant 2$, we have

$$
|\Lambda|\left(\alpha(d) 2^{1+2 d / \kappa}(1-p)\right)^{(\kappa c \ln |\Lambda|) / 2 d} \leqslant \frac{1}{|\Lambda|^{c}} .
$$

Notice that the constant $\kappa$ doesn't depend on $k$. Therefore, the inequalities

$$
\left\{\left(H_{k, m}\right),\left(G_{k, m}\right): 1 \leqslant k+m \leqslant c \ln |\Lambda|\right\}
$$

are all satisfied for $p \geqslant \tilde{p}$ and this $\kappa$. This concludes the induction. 


\section{The law of an edge far from a cut}

We now show the Theorem 1.3 with the help of Proposition 6.3.

Proof of Theorem 1.3: Since $\mu$ is the stationary distribution of $\left(X_{t}, Y_{t}\right)_{t \in \mathbb{N}}$, we can choose a time $t$ and show the result for the configuration $\left(X_{t}, Y_{t}\right)$. For a time $r \in \mathbb{N}$ and a distance $\ell>0$, we introduce the events

$$
\bar{D}(r, \ell)=\left\{\exists C \in \mathcal{C}_{r}, d(e, C) \geqslant \ell\right\}
$$

and

$$
\left.D(r, \ell)=\{\forall \theta \in] r, r+|\Lambda|], \exists C_{\theta} \in \mathcal{C}_{\theta}, d\left(e, C_{\theta}\right) \geqslant \ell\right\} .
$$

We have to estimate the probability

$$
P_{\mu}\left(e \in \mathcal{I}_{t} \mid \bar{D}\left(t, \kappa^{\prime} c^{2} \ln ^{2}|\Lambda|\right)\right)
$$

where $\kappa^{\prime}$ is a constant which will be determined later. For the moment, we can simply consider a large $\kappa^{\prime}$. We notice first that, on the event $\bar{D}\left(t, \kappa^{\prime} c^{2} \ln ^{2}|\Lambda|\right)$, there is a cut which is disjoint from $e$, so the edge $e$ cannot be pivotal, thus

$$
P_{\mu}\left(e \in \mathcal{I}_{t} \mid \bar{D}\left(t, \kappa^{\prime} c^{2} \ln ^{2}|\Lambda|\right)\right)=P_{\mu}\left(e \in \mathcal{I}_{t} \backslash \mathcal{P}_{t} \mid \bar{D}\left(t, \kappa^{\prime} c^{2} \ln ^{2}|\Lambda|\right)\right)
$$

We consider the last time when $e$ is pivotal, i.e., the time $t-s$ defined by

$$
s=\inf \left\{r \geqslant 0: e \in \mathcal{P}_{t-r}\right\} .
$$

On the interval $] t-s, t]$, the edge $e$ is not pivotal and it remains in the interface. Therefore, this edge is not modified during this interval, so we have

$$
P_{\mu}\left(e \in \mathcal{I}_{t} \mid \bar{D}\left(t, \kappa^{\prime} c^{2} \ln ^{2}|\Lambda|\right)\right) \leqslant P_{\mu}\left(\begin{array}{c}
\exists s \geqslant 0, e \in \mathcal{P}_{t-s} \\
\forall r \in] t-s, t] \\
e \notin \mathcal{P}_{r}, E_{r} \neq e
\end{array} \mid \bar{D}\left(t, \kappa^{\prime} c^{2} \ln ^{2}|\Lambda|\right)\right)
$$

The events appearing in this probability concern only the process $\left(E_{t}\right)_{t \in \mathbb{N}}$ and the process $\left(Y_{t}\right)_{t \in \mathbb{N}}$. These processes are both reversible. By reversing the time, we obtain that

$$
\begin{aligned}
P_{\mu}\left(\begin{array}{c}
\exists s \geqslant 0, e \in \mathcal{P}_{t-s} \\
\forall r \in] t-s, t] \\
e \notin \mathcal{P}_{r}, E_{r} \neq e
\end{array} \mid \bar{D}\left(t, \kappa^{\prime} c^{2} \ln ^{2}|\Lambda|\right)\right)= & \\
& P_{\mu}\left(\begin{array}{c}
\exists s \geqslant 0, e \in \mathcal{P}_{t+s} \\
\forall r \in] t, t+s] \\
e \notin \mathcal{P}_{r}, E_{r} \neq e
\end{array} \mid \bar{D}\left(t, \kappa^{\prime} c^{2} \ln ^{2}|\Lambda|\right)\right) .
\end{aligned}
$$

Notice that the sequence $\left(E_{r}\right)_{t<r \leqslant t+s}$ is independent of the configuration $Y_{t}$. We estimate first the probability that the interval $] t, t+s]$ is too large. More precisely, we will show that $s$ is at most of order $|\Lambda| \ln |\Lambda|$. Let $c \geqslant 1$ be a constant. We have

$$
\begin{aligned}
& P_{\mu}\left(\begin{array}{c|c}
\exists s \geqslant c|\Lambda| \ln |\Lambda|, e \in \mathcal{P}_{t+s} & \\
\forall r \in] t, t+s] & \bar{D}\left(t, \kappa^{\prime} c^{2} \ln ^{2}|\Lambda|\right) \\
e \notin \mathcal{P}_{r}, E_{r} \neq e &
\end{array}\right) \leqslant \\
& P_{\mu}\left(\begin{array}{c}
\forall r \in] t, t+c|\Lambda| \ln |\Lambda|] \\
E_{r} \neq e
\end{array}\right) \leqslant\left(1-\frac{1}{|\Lambda|}\right)^{c|\Lambda| \ln |\Lambda|} \leqslant \frac{1}{|\Lambda|^{c}}
\end{aligned}
$$

We now consider the case where $s<c|\Lambda| \ln |\Lambda|$. We split the interval $[t, t+s]$ into subintervals of length $|\Lambda|$. We set, for $0 \leqslant i<s /|\Lambda|$,

$$
t_{i}=t+i|\Lambda|
$$

Let us distinguish two cases according to the positions of the cuts during the time interval $\left.] t, t_{1}\right]$. We consider a constant $\kappa>0$ which will be chosen later. If the event $D\left(t, \kappa^{\prime} c^{2} \ln ^{2}|\Lambda|-\kappa c \ln |\Lambda|\right)$ 
doesn't occur, then there exists a time $\left.\tau \in] t, t_{1}\right]$ and a cut of $\mathcal{C}_{\tau}$ which visits at least an edge $f$ at distance less than $\kappa^{\prime} c^{2} \ln ^{2}|\Lambda|-\kappa c \ln |\Lambda|$ from $e$. Therefore, for a $s<c|\Lambda| \ln |\Lambda|$ fixed, we have

$$
\begin{aligned}
& P_{\mu}\left(e \in \mathcal{P}_{t+s} \mid \bar{D}\left(t, \kappa^{\prime} c^{2} \ln ^{2}|\Lambda|\right)\right) \leqslant \\
& P_{\mu}\left(\begin{array}{c}
\left.\exists \tau \in] t, t_{1}\right], \exists C_{\tau} \in \mathcal{C}_{\tau}, \exists f \in C_{\tau} \\
d(e, f) \leqslant \kappa^{\prime} c^{2} \ln ^{2}|\Lambda|-\kappa c \ln |\Lambda|
\end{array} \mid \bar{D}\left(t, \kappa^{\prime} c^{2} \ln ^{2}|\Lambda|\right)\right)+ \\
& \quad P_{\mu}\left(\begin{array}{c}
e \in \mathcal{P}_{t+s} \\
D\left(t, \kappa^{\prime} c^{2} \ln ^{2}|\Lambda|-\kappa c \ln |\Lambda|\right)
\end{array} \mid \bar{D}\left(t, \kappa^{\prime} c^{2} \ln ^{2}|\Lambda|\right)\right) .
\end{aligned}
$$

We estimate the first probability with the help of Corollary 6.1. This case is illustrated in Figure 6.7 but this time with the radius of the circles taken to be $\kappa^{\prime} c^{2} \ln ^{2}|\Lambda|$ and $\kappa^{\prime} c^{2} \ln ^{2}|\Lambda|-\kappa c \ln |\Lambda|$. There is a $\tilde{p}<1$, such that, for $p \geqslant \tilde{p}$ and $\kappa>0$, for any $c \geqslant 2,0<\tau \leqslant|\Lambda|$ and an edge $f$ at distance less than $\kappa^{\prime} c^{2} \ln ^{2}|\Lambda|-\kappa c \ln |\Lambda|$ from $e$, we have

$$
P_{\mu}\left(\exists C_{\tau} \in \mathcal{C}_{\tau}, f \in C_{\tau} \mid \bar{D}\left(t, \kappa^{\prime} c^{2} \ln ^{2}|\Lambda|\right)\right) \leqslant \frac{1}{|\Lambda|^{c+2}} .
$$

Therefore, the following inequality holds:

$$
\begin{aligned}
P_{\mu}\left(\begin{array}{c}
\left.\exists \tau \in] t, t_{1}\right], \exists C_{\tau} \in \mathcal{C}_{\tau}, \exists f \in C_{\tau} \\
d(e, f) \leqslant \kappa^{\prime} c^{2} \ln ^{2}|\Lambda|-\kappa c \ln |\Lambda|
\end{array} \mid \bar{D}\left(t, \kappa^{\prime} c^{2} \ln ^{2}|\Lambda|\right)\right) \leqslant \\
\sum_{\left.\tau \in] t, t_{1}\right]} P_{\mu}\left(\exists C_{\tau} \in \mathcal{C}_{\tau}, d\left(e, C_{\tau}\right) \leqslant \kappa^{\prime} c^{2} \ln ^{2}|\Lambda|-\kappa c \ln |\Lambda| \mid \bar{D}\left(t, \kappa^{\prime} c^{2} \ln ^{2}|\Lambda|\right)\right) \leqslant \frac{2 d}{|\Lambda|^{c}} .
\end{aligned}
$$

We then obtain

$$
P_{\mu}\left(e \in \mathcal{P}_{t+s} \mid \bar{D}\left(t, \kappa^{\prime} \ln ^{2}|\Lambda|\right)\right) \quad \leqslant \quad \frac{2 d}{|\Lambda|^{c}}+P_{\mu}\left(e \in \mathcal{P}_{t+s} \mid \begin{array}{c}
D\left(t, \kappa^{\prime} \ln ^{2}|\Lambda|-\kappa \ln |\Lambda|\right) \\
\bar{D}\left(t, \kappa^{\prime} \ln ^{2}|\Lambda|\right)
\end{array}\right) .
$$

Starting from this inequality, we apply Proposition 6.3 and repeat the previous argument at the times $t_{i}, 0 \leqslant i<s /|\Lambda|$. By iteration, we obtain that, for any $n<s /|\Lambda|$ and $|\Lambda| \geqslant 12(2 \kappa d)^{d}$,

$$
\begin{aligned}
& P_{\mu}\left(e \in \mathcal{P}_{t+s} \mid \bar{D}\left(t, \kappa^{\prime} c^{2} \ln ^{2}|\Lambda|\right)\right) \leqslant
\end{aligned}
$$

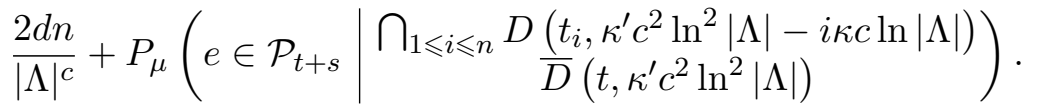

We consider this inequality with $n=\lfloor s /|\Lambda|\rfloor<c \ln |\Lambda|$ :

$$
\begin{aligned}
& P_{\mu}\left(e \in \mathcal{P}_{t+s} \mid \bar{D}\left(t, \kappa^{\prime} c^{2} \ln ^{2}|\Lambda|\right)\right) \leqslant \\
& \frac{2 d c \ln |\Lambda|}{|\Lambda|^{c}}+P_{\mu}\left(e \in \mathcal{P}_{t+s} \mid \bigcap_{1 \leqslant i<s /|\Lambda|} \frac{D\left(t_{i}, \kappa^{\prime} c^{2} \ln ^{2}|\Lambda|-i \kappa c \ln |\Lambda|\right)}{D\left(t, \kappa^{\prime} c^{2} \ln ^{2}|\Lambda|\right)}\right) .
\end{aligned}
$$

We notice that $s-|\Lambda|\lfloor s /|\Lambda|\rfloor<|\Lambda|$ and there exists a $\kappa^{\prime}>0$ such that

$$
\kappa^{\prime} c^{2} \ln ^{2}|\Lambda|-\kappa c \ln |\Lambda|\lfloor s /|\Lambda|\rfloor \geqslant \kappa c \ln |\Lambda| .
$$

We can apply again Proposition 6.3 at time $t_{n}$ and we get

$$
P_{\mu}\left(e \in \mathcal{P}_{t+s} \mid \begin{array}{c}
\bigcap_{1 \leqslant i<c \ln |\Lambda|} \frac{D\left(t_{i}, \kappa^{\prime} c^{2} \ln ^{2}|\Lambda|-i \kappa c \ln |\Lambda|\right)}{D}\left(t, \kappa^{\prime} c^{2} \ln ^{2}|\Lambda|\right)
\end{array}\right) \leqslant \frac{1}{|\Lambda|^{c}} .
$$

Finally, we obtain the following upper bound for (7.3):

$$
P_{\mu}\left(e \in \mathcal{P}_{t+s} \mid \bar{D}\left(t, \kappa^{\prime} c^{2} \ln ^{2}|\Lambda|\right)\right) \leqslant \frac{2 d c \ln |\Lambda|+1}{|\Lambda|^{c}} .
$$


We sum over the choices of $s<c|\Lambda| \ln |\Lambda|$ and we combine with (7.2). We obtain

$$
P_{\mu}\left(\begin{array}{c}
\exists s \geqslant 0, e \in \mathcal{P}_{t+s} \\
\forall r \in] t, t+s] \\
e \notin \mathcal{P}_{r}, E_{r} \neq e
\end{array} \mid \bar{D}\left(t, \kappa^{\prime} c^{2} \ln ^{2}|\Lambda|\right)\right) \leqslant \frac{1+c|\Lambda| \ln |\Lambda|+2 d|\Lambda| c^{2} \ln ^{2}|\Lambda|}{|\Lambda|^{c}} .
$$

For $|\Lambda|>4+c+2 d c^{2}+12(2 \kappa d)^{d}$, we have $\ln |\Lambda| \leqslant|\Lambda|$ and thus

$$
\frac{1+c|\Lambda| \ln |\Lambda|+2|\Lambda| d c^{2} \ln ^{2}|\Lambda|}{|\Lambda|^{c}} \leqslant \frac{1+c+2 d c^{2}}{|\Lambda|^{c-3}} \leqslant \frac{1}{|\Lambda|^{c-4}}
$$

Therefore, there exists a $\tilde{p}<1$ and a $\kappa^{\prime}>0$ such that for $p \geqslant \tilde{p}$, for any $c \geqslant 2$, we have

$$
P_{\mu}\left(e \in \mathcal{I}_{t} \backslash \mathcal{P}_{t} \mid \bar{D}\left(t, \kappa^{\prime} c^{2} \ln ^{2}|\Lambda|\right)\right) \leqslant \frac{1}{|\Lambda|^{c-4}} .
$$

Since $(c+4)^{2} / c^{2} \leqslant 25$ for $c \geqslant 1$, by replacing $\kappa^{\prime}$ by $25 \kappa^{\prime}$ in the probability, we can replace $1 /|\Lambda|^{c-4}$ by $1 /|\Lambda|^{c}$. Hence the desired result.

\section{The construction of the impatient STP}

We will construct a STP which connects an edge $e \in \mathcal{P}_{t}$ at time $t$ and the set $\mathcal{P}_{s} \cup \mathcal{I}_{s}$ at time $s<t$. Before starting the construction, we define first some relevant properties of a STP, which will be enjoyed by our construction.

Definition 8.1. A STP $\left(e_{1}, t_{1}\right), \ldots,\left(e_{n}, t_{n}\right)$ is impatient if every time-change is ended by an edge which is updated, i.e.,

$$
\forall i \in\{1, \ldots, n-2\} \quad e_{i}=e_{i+1} \Rightarrow E_{t_{i+1}+1}=e_{i+1} .
$$

Definition 8.2. A STP $\left(e_{1}, t_{1}\right), \ldots,\left(e_{n}, t_{n}\right)$ is called $X$-closed-moving (resp. $Y$-closed-moving) if all the edges which are not time-change edges are closed in $X$ (resp. in $Y$ ), i.e.,

$$
\forall i \in\{1, \ldots, n-1\} \quad e_{i} \neq e_{i+1} \Rightarrow X_{t_{i}}\left(e_{i}\right)=0 \quad\left(\text { resp. } Y_{t_{i}}\left(e_{i}\right)=0\right) .
$$

We now construct a specific STP satisfying some of these properties.

Proposition 8.3. Let $s<t$ be two times and $e \in \mathcal{P}_{t}$. There exists a decreasing simple impatient $S T P$ which connects the time-edge $(e, t)$ to an edge of the set $\mathcal{P}_{s} \cup \mathcal{I}_{s} \backslash\{e\}$ at time $s$ or an edge $f$ intersecting the boundary of $\Lambda$ after time s. Moreover this STP is X-closed-moving except on the edge e.

Proof: The proof of this proposition is done in two steps. The first step is to construct a STP which connects certain edges. In the second step, we modify the STP obtained in the first step to get a simple and impatient STP.

Step 1. At time $t$, the edge $e$ belongs to a cut. Therefore, there exists a path $\gamma_{1}$ which connects $e$ to the boundary of $\Lambda$. We start at the edge $e$ and we follow the path $\gamma_{1}$. If the path $\gamma_{1}$ does not encounter an edge $f \in \mathcal{I}_{t} \cup \mathcal{P}_{t-1} \backslash\{e\}$ then the STP

$$
(e, t),\left(\gamma_{1}, t\right)
$$

connects $e$ to the boundary of $\Lambda$, where the notation $(\rho, t)$, for a path $\rho=\left(e_{i}\right)_{1 \leqslant i \leqslant n}$ and a time $t$, means the sequence of time-edges $\left(e_{i}, t\right)_{1 \leqslant i \leqslant n}$. Suppose next that there exists an edge of $\mathcal{I}_{t} \cup \mathcal{P}_{t-1} \backslash\{e\}$ in $\gamma_{1}$. We enumerate the edges of $\gamma_{1}$ in the order they are visited when starting from $e$ and we consider the first edge $e_{1}$ in $\gamma_{1}$ which belongs to the set $\mathcal{I}_{t} \cup \mathcal{P}_{t-1}$. We denote by $\rho_{1}$ the sub-path of $\gamma_{1}$ visited between $e$ and $e_{1}$. We then consider the time $\eta(t)$ defined as follows:

$$
\eta(t)=\max \left\{r<t: X_{r}\left(e_{1}\right)=Y_{r}\left(e_{1}\right)\right\} .
$$


Since $e_{1} \in \mathcal{I}_{t}$, the time $\eta(t)$ when it becomes an edge of the interface is strictly less than $t$ and if $e_{1} \in \mathcal{P}_{t-1} \backslash \mathcal{I}_{t}$, we have $\eta(t) \leqslant t-1$. In both cases, we have $\eta(t)<t$ and the edge $e_{1}$ is closed in $X_{\eta(t)}$. If the time $\eta(t)$ is before the time $s$ then, at time $s$, the edge $e_{1}$ belongs to the set $\mathcal{I}_{s} \cup \mathcal{P}_{s} \backslash\{e\}$. Therefore the STP

$$
(e, t),(\rho, t),\left(e_{1}, t\right),\left(e_{1}, s\right)
$$

satisfies the conditions in the proposition. If we have $\eta(t)>s$, then we repeat the above argument starting from the edge $e_{1}$ at time $\eta(t)$. We obtain either a path $\gamma_{2}$ which connects $e_{1}$ to the boundary of $\Lambda$ at time $\eta(t)$ or a path $\rho_{2}$ which connects $e_{1}$ to an edge $e_{2} \in \mathcal{I}_{\eta(t)} \cup \mathcal{P}_{\eta(t)-1} \backslash\{e\}$ and a time $\eta^{2}(t)<\eta(t)$. We proceed in this way until we reach a time edge $\left(e_{k}, \eta^{k}(t)\right)$ with $\eta^{k}(t) \leqslant s$. Since $\eta(t)<t$, the sequence of times

$$
\eta(t), \eta^{2}(t), \ldots, \eta^{k}(t)
$$

decreases strictly through this procedure and this procedure terminates after a finite number of iterations. The concatenation of the paths obtained at the end of the procedure,

$$
(e, t),\left(\rho_{1}, t\right),\left(e_{1}, \eta(t)\right), \ldots,\left(\rho^{k}, \eta^{k-1}(t)\right),\left(f_{k}, s\right),
$$

connects $e$ to an edge of $\mathcal{P}_{s} \cup \mathcal{I}_{s} \backslash\{e\}$. Since the sequence $\left(\eta^{i}(t)\right)_{1 \leqslant i \leqslant k}$ is decreasing, this is a decreasing STP. Each time when the STP meets an edge of $\mathcal{I}$ which is different from $e$, there is a time change to the time before it opened in $X$, therefore each movement in space except on the edge $e$ is done through a closed edge in $X$ and the STP is $X$-closed-moving.

Step 2. We use two iterative procedures to transform the STP in the step 1 into a simple and impatient STP. To get a simple STP, we use the same procedure as in the proof of Proposition 4.4. Let us denote by $\left(e_{i}, t_{i}\right)_{0 \leqslant i \leqslant N}$ the STP obtained previously. Starting with the edge $e_{0}$, we examine the rest of the edges one by one. Let $i \in\{0, \ldots, N\}$. Suppose that the edges $e_{0}, \ldots, e_{i-1}$ have been examined and let us focus on $e_{i}$. We encounter three cases:

- For every index $j \in\{i+1, \ldots, N\}$, we have $e_{j} \neq e_{i}$. Then, we don't modify anything and we start examining the edge $e_{i+1}$.

- There is an index $j \in\{i+1, \ldots, N\}$ such that $e_{i}=e_{j}$, but for the first index $k>i+1$ such that $e_{i}=e_{k}$, there is a time $\left.\alpha \in\right] t_{k}, t_{i}$ [ when $X_{\alpha}\left(e_{i}\right)=1$. Then we don't modify anything and we start examining the next edge $e_{i+1}$.

- There is an index $j \in\{i+1, \ldots, N\}$ such that $e_{i}=e_{j}$ and for the first index $k>i+1$ such that $e_{i}=e_{k}$, we have $X_{\alpha}\left(e_{i}\right)=0$ for all $\left.\alpha \in\right] t_{k}, t_{i}$ [. In this case, we remove all the time-edges whose indices are strictly between $i$ and $k$. We then have a simple time change between $t_{i}$ and $t_{k}$ on the edge $e_{i}$. We continue the procedure from the index $k$.

The STP becomes strictly shorter after every modification (we remove systematically the consecutive time changes if there is any), and the procedure will end after a finite number of modifications. We obtain in the end a simple path in $X$. Since the procedure doesn't change the order of the times $t_{i}$, we still have a decreasing path. In order to obtain an impatient STP, we modify the simple decreasing STP obtained above and we use another iterative procedure as follows. We denote again by $\left(e_{i}, t_{i}\right)_{0 \leqslant i \leqslant n}$ the simple STP obtained above. We start by examining the time-edge $\left(e_{0}, t_{0}\right)$ and then the rest of the time edges of the STP one by one as illustrated in the Figure 8.8. Suppose that we have examined the indices $i<k$ and that we are checking the index $k$. If the edge $e_{k+1}$ is different from $e_{k}$, we don't modify the STP at this stage and we continue the procedure from $\left(e_{k+1}, t_{k+1}\right)$. If the edge $e_{k+1}$ is equal to $e_{k}$, then the time-edge $\left(e_{k}, t_{k}\right)$ belongs to a time change. Since the STP is $X$-closed-moving, then the edge $e_{k+1}$ is closed at time $t_{k+1}$. Let $[\alpha, \beta]$ be the biggest interval containing $t_{k+1}$ during which the edge $e_{k+1}$ is closed in $X$. If $\beta \geqslant t_{k}$ and $e_{k+2} \neq e_{k+3}$, we replace the sub-sequence

by

$$
\left(e_{k}, t_{k}\right),\left(e_{k+1}, t_{k+1}\right),\left(e_{k+2}, t_{k+2}\right)
$$

$$
\left(e_{k}, t_{k}\right),\left(e_{k+2}, t_{k}\right),\left(e_{k+2}, t_{k+2}\right),
$$




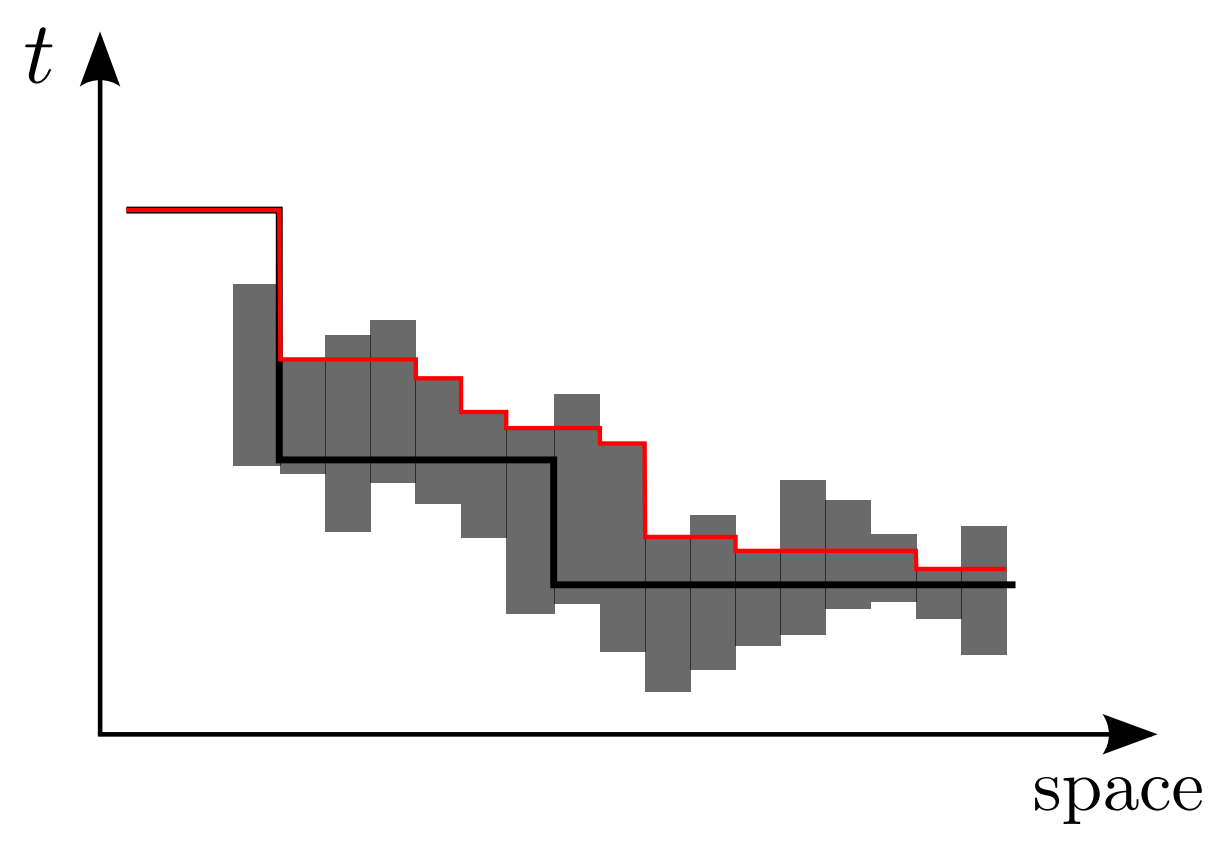

Figure 8.8. An impatient modification (in red) of a STP (in black) according to the intervals when each edge is closed (in gray)

and we continue the modification from the time-edge $\left(e_{k+2}, t_{k}\right)$. If $\beta \geqslant t_{k}$ and $e_{k+2}=e_{k+3}$ we replace the sequence

$$
\left(e_{k}, t_{k}\right),\left(e_{k+1}, t_{k+1}\right),\left(e_{k+2}, t_{k+2}\right),\left(e_{k+3}, t_{k+3}\right)
$$

by

$$
\left(e_{k}, t_{k}\right),\left(e_{k+2}, t_{k}\right),\left(e_{k+2}, t_{k+3}\right)
$$

and we continue the modification from the time-edge $\left(e_{k+2}, t_{k}\right)$. If $\beta<t_{k}$ and $e_{k+2} \neq e_{k+3}$, we replace $\left(e_{k}, t_{k}\right),\left(e_{k+1}, t_{k+1}\right)$ by

$$
\left(e_{k}, t_{k}\right),\left(e_{k}, \beta\right),\left(e_{k+2}, \beta\right) .
$$

If $\beta<t_{k}$ and $e_{k+2}=e_{k+3}$, we replace $\left(e_{k}, t_{k}\right),\left(e_{k+1}, t_{k+1}\right),\left(e_{k+2}, t_{k+2}\right)$ by

$$
\left(e_{k}, t_{k}\right),\left(e_{k}, \beta\right),\left(e_{k+2}, \beta\right)
$$

and we continue the STP at the time-edge $\left(e_{k+2}, \beta\right)$. The STP obtained after the modification procedure is decreasing, $X$-closed-moving and impatient. Moreover, between two consecutive visits of an edge $f$ of the STP, there exists a time when the edge $f$ is open. Therefore, this STP is also simple.

\section{Exponential decay of the new STP}

We show here that the set $\mathcal{P} \cup \mathcal{I}$ cannot move too fast. Typically, during an interval of size $|\Lambda| \ln |\Lambda|$, the set $\mathcal{P} \cup \mathcal{I}$ can at most move a distance of order $\ln |\Lambda|$. This result relies on an estimate for the STP constructed in Proposition 8.3 which we state in the following proposition. 
Lemma 9.1. Let $e$ be an edge in $\Lambda$ and $\ell \in \mathbb{N}^{*}$. Let $\left(\varepsilon_{1}, \ldots, \varepsilon_{n}\right)$ be a sequence of edges such that $\left|\operatorname{support}\left(\varepsilon_{1}, \ldots, \varepsilon_{n}\right)\right|=\ell$. We have the following inequality:

$$
\begin{aligned}
\exists \tilde{p}<1 \quad \forall p \geqslant \tilde{p} \quad \forall s, t \quad 0<t-s \leqslant \ell|\Lambda| \\
\qquad P_{\mu}\left(\begin{array}{c}
\exists \gamma \text { decreasing simple impatient } \\
X \text {-closed-moving STP except on } e, \\
\gamma \text { starts from }(e, t) \text { and ends after } s, \\
\operatorname{space}(\gamma)=\left(\varepsilon_{1}, \ldots, \varepsilon_{n}\right)
\end{array}\right) \leqslant\left(1+\frac{1}{|\Lambda|}\right)^{\ell|\Lambda|}(4-4 p)^{n} .
\end{aligned}
$$

Proof: Let us fix a STP $\gamma$ satisfying the conditions stated in the probability. We denote by $\left(e_{i}, t_{i}\right)_{i \in I}$ the sequence of the time-edges of $\gamma$. We denote by $k$ the number of the time changes in $\gamma$ and by $T$ the set of the indices of the time changes, i.e.,

$$
T=\left\{i \in I: e_{i}=e_{i+1}, \quad t_{i} \neq t_{i+1}\right\} .
$$

We shall obtain an upper bound of the probability

$$
P\left(\begin{array}{c}
\left(e_{i}, t_{i}\right)_{i \in I} \text { is a decreasing simple impatient } \\
X \text {-closed-moving STP except on } e
\end{array}\right),
$$

which depends only upon the integer $n$ and the number of time changes $k$. In order to bound the probability appearing in the lemma, we shall sum over the choices of the set of the $k$ times, denoted by $K$, in the interval $\{s, \ldots, t\}$, over the choices of set of the $k$ edges, denoted by $A$, where the time changes occur and the number $k$ from 1 to $n$. The probability appearing in the lemma is less or equal than

$$
\sum_{1 \leqslant k \leqslant n} \sum_{\substack{A \subset\{1, \ldots, n\} \\
|A|=k}} \sum_{\substack{\mathcal{A}\{s, \ldots, t\} \\
|K|=k}} P\left(\begin{array}{c}
\left(e_{i}, t_{i}\right)_{i \in I} \text { is a decreasing simple impatient } \\
X \text {-closed-moving STP except on } e
\end{array}\right) .
$$

Let us obtain an upper bound for this probability. The STP is impatient and $X$-closed-moving, therefore for any $i \in T$, the edge $e_{i+1}$ becomes open at time $t_{i+1}+1$. Moreover, the STP is simple, thus for any pair of indices $(p, q) \in I \backslash T$, if $e_{p}=e_{q}$ and $t_{p}>t_{q}$, there exists a time $\left.r \in\right] t_{q}, t_{p}[$, such that the edge $e_{p}$ is open at time $r$. We can rewrite the probability inside the sum as

$$
P_{\mu}\left(\begin{array}{c}
\forall i \in T \quad E_{t_{i+1}+1}=e_{i+1} \\
\forall i \in I \backslash T \quad X_{t_{i}}\left(e_{i}\right)=0 \\
\forall p, q \in I \backslash T \text { s.t. } e_{p}=e_{q}, t_{p}>t_{q} \\
\exists r \in] t_{q}, t_{p}\left[\quad X_{r}\left(e_{p}\right)=1\right.
\end{array}\right) .
$$

Since the times $t_{i}$ are fixed, this probability can be factorised as a product over the edges. In fact, the event in the probability depends only on the process $\left(X_{t}\right)_{t \in \mathbb{N}}$. We introduce, for an edge $f \subset \Lambda$, the subset $J(f)$ of $I$ :

$$
J(f)=\left\{i \in I: e_{i}=f\right\} .
$$

Let us denote by $S$ the set $\operatorname{support}(\gamma)$. The previous probability is less or equal than

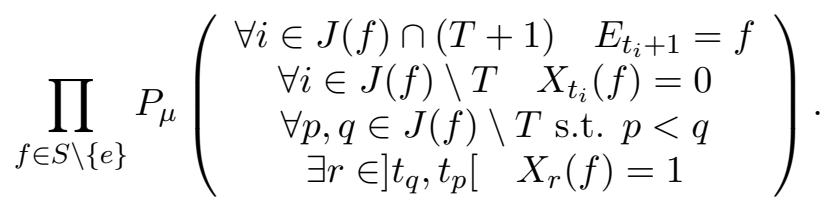

Let us consider one term of the product. For a fixed edge $f$, we can order the set $\left\{t_{i}: i \in J(f) \backslash T\right\}$ in an increasing sequence $\left(\tau_{i}\right)_{1 \leqslant i \leqslant m_{f}}$, where $m_{f}=|J(f) \backslash T|$. Let us denote by $T(f)$ the set of the indices among $\left\{1, \ldots, m_{f}\right\}$ which correspond to the end of a time change, i.e., the set corresponding to $J(f) \cap(T+1)$ before the reordering. Since the STP is simple, between two consecutive visits at times $\tau_{i}$ and $\tau_{i+1}$ of $f$, there is a time $\theta_{i}$ when $f$ is open. Moreover the STP is impatient, so for each 
index $i \in T(f)$, the edge $f$ becomes open at time $\tau_{i}+1$. Therefore, each term of the product (9.4) is less or equal than

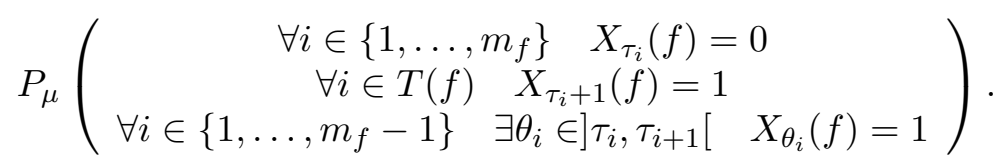

In order to simplify the notations, we define, for a time $r$, the event

$$
\mathcal{E}(r)=\left\{\begin{array}{c}
\forall i \in\left\{1, \ldots, m_{f}\right\} \text { such that } \tau_{i} \leqslant r \quad X_{\tau_{i}}(f)=0 \\
\forall i \in T(f) \text { such that } \tau_{i}+1 \leqslant r \quad X_{\tau_{i}+1}(f)=1 \\
\forall i \in\left\{1, \ldots, m_{f}-1\right\} \text { such that } \tau_{i} \leqslant r \\
\left.\exists \theta_{i} \in\right] \tau_{i}, \tau_{i+1}\left[X_{\theta_{i}}(f)=1\right.
\end{array}\right\} .
$$

The status of the edge $f$ in the process $\left(X_{t}\right)_{t \in \mathbb{N}}$ evolves according to a Markov chain on $\{0,1\}$. The sequence $\left(\tau_{i}\right)_{1 \leqslant i \leqslant m_{f}}$ being fixed, if $m_{f} \in T(f)$, we condition 9.5 by the events before time $\tau_{m_{f}}$, we have

$$
\begin{aligned}
& P_{\mu}\left(\begin{array}{c}
\forall i \in\left\{1, \ldots, m_{f}\right\} \quad X_{\tau_{i}}(f)=0 \\
\forall i \in T(f) \quad X_{\tau_{i}+1}(f)=1, \quad m_{f} \in T(f) \\
\left.\forall i \in\left\{1, \ldots, m_{f}-1\right\} \quad \exists \theta_{i} \in\right] \tau_{i}, \tau_{i+1}\left[\quad X_{\theta_{i}}(f)=1\right.
\end{array}\right)= \\
& P_{\mu}\left(X_{\tau_{m_{f}}+1}(f)=1 \mid \mathcal{E}\left(\tau_{m_{f}}\right)\right) P_{\mu}\left(\mathcal{E}\left(\tau_{m_{f}}\right)\right) \leqslant \frac{P_{\mu}\left(\mathcal{E}\left(\tau_{m_{f}}\right)\right)}{|\Lambda|} .
\end{aligned}
$$

If $m_{f} \notin T(f)$, the probability

$$
P_{\mu}\left(\begin{array}{c}
\forall i \in\left\{1, \ldots, m_{f}\right\} \quad X_{\tau_{i}}(f)=0 \\
\forall i \in T(f) \quad X_{\tau_{i}+1}(f)=1, \quad m_{f} \notin T(f) \\
\left.\forall i \in\left\{1, \ldots, m_{f}-1\right\} \quad \exists \theta_{i} \in\right] \tau_{i}, \tau_{i+1}\left[\quad X_{\theta_{i}}(f)=1\right.
\end{array}\right)
$$

is equal to $P_{\mu}\left(\mathcal{E}\left(\tau_{m_{f}}\right)\right)$. We then condition $P_{\mu}\left(\mathcal{E}\left(\tau_{m_{f}}\right)\right)$ by the events before time $\tau_{m_{f}-1}$. We shall distinguish two cases according to whether $m_{f}-1$ belongs to $T(f)$ or not. If $m_{f}-1 \in T(f)$, we have

$$
P_{\mu}\left(\mathcal{E}\left(\tau_{m_{f}}\right)\right)=P_{\mu}\left(\begin{array}{c|c}
X_{\tau_{m_{f}}}(f)=0 \\
X_{\tau_{m_{f}-1}+1}(f)=1 & \mathcal{E}\left(\tau_{m_{f}-1}\right)
\end{array}\right) P_{\mu}\left(\mathcal{E}\left(\tau_{m_{f}-1}\right)\right),
$$

and if $m_{f}-1 \notin T(f)$, we have

$$
P_{\mu}\left(\mathcal{E}\left(\tau_{m_{f}}\right)\right)=P_{\mu}\left(\begin{array}{c}
X_{\tau_{m_{f}}}(f)=0 \\
\left.\exists \theta_{m_{f}} \in\right] \tau_{m_{f}-1}, \tau_{m_{f}}[ \\
X_{\theta_{m_{f}}}(f)=1
\end{array} \mid \mathcal{E}\left(\tau_{m_{f}-1}\right)\right) P_{\mu}\left(\mathcal{E}\left(\tau_{m_{f}-1}\right)\right) .
$$

We condition successively the event $P_{\mu}\left(\mathcal{E}\left(\tau_{i}\right)\right)$ by $\mathcal{E}\left(\tau_{i-1}\right)$, we obtain

$$
\begin{aligned}
P_{\mu}\left(\mathcal{E}\left(\tau_{m_{f}}\right)\right)=P_{\mu}\left(\mathcal{E}\left(\tau_{1}\right)\right) & \prod_{1 \leqslant i<m_{f}, i \in T(f)} P_{\mu}\left(\begin{array}{r|r}
X_{\tau_{i+1}}(f)=0 \\
X_{\tau_{i}+1}(f)=1
\end{array}\right. \\
& \times \prod_{1 \leqslant i<m_{f}, i \notin T(f)} P_{\mu}\left(\begin{array}{c}
X_{\tau_{i+1}}(f)=0 \\
\left.\exists \theta_{i+1} \in\right] \tau_{i}, \tau_{i+1}[ \\
X_{\theta_{i}}(f)=1
\end{array}\right.
\end{aligned}
$$

By the Markov property, each term in the second product is equal to

$$
P_{\mu}\left(\begin{array}{c|c}
X_{\tau_{i+1}}(f)=0 \\
\left.\exists \theta_{i} \in\right] \tau_{i}, \tau_{i+1}[ & X_{\tau_{i}}(f)=0 \\
X_{\theta_{i+1}}(f)=1 &
\end{array}\right)
$$


Since this probability is invariant by translation in time, it is equal to

$$
P_{0}\left(\begin{array}{c}
X_{\tau^{\prime}}(f)=0 \\
\exists \theta \in] 0, \tau^{\prime}[ \\
X_{\theta}(f)=1
\end{array}\right)
$$

where we have set $\tau^{\prime}=\tau_{i+1}-\tau_{i}$ and $P_{0}$ is the law of the Markov chain $\left(X_{t}(f)\right)_{t \in \mathbb{N}}$ starting from a closed edge. By considering the stopping time $\theta^{\prime}$ defined as the first time after 0 when $f$ is open, we have by the strong Markov property

$$
P_{0}\left(\begin{array}{c}
X_{\tau^{\prime}}(f)=0 \\
\exists \theta \in] 0, \tau^{\prime}[ \\
X_{\theta}(f)=1
\end{array}\right) \leqslant P_{0}\left(X_{\tau^{\prime}}(f)=0 \mid X_{\theta^{\prime}}(f)=1\right)=P_{1}\left(X_{\tau^{\prime}-\theta^{\prime}}(f)=0\right) .
$$

Notice that for $r \geqslant 1$, we have

$$
P_{1}\left(X_{r}(f)=0\right) \leqslant P_{\mu}\left(X_{r}(f)=0\right)=1-p .
$$

Therefore we have

$$
P_{0}\left(\begin{array}{c}
X_{\tau^{\prime}}(f)=0 \\
\exists \theta \in] 0, \tau^{\prime}[ \\
X_{\theta}(f)=1
\end{array}\right) \leqslant 1-p
$$

As for the probabilities in the first product of (9.6), we can also replace $\mathcal{E}\left(\tau_{i}\right)$ by $\left\{X_{\tau_{i}}(f)=0\right\}$ in the conditioning. The difference between the previous case is that we have directly $\theta^{\prime}=1$, since $X_{\tau_{i}+1}(f)=1$. We have

$$
P_{\mu}\left(\begin{array}{c|c}
X_{\tau_{i+1}}(f)=0 \\
X_{\tau_{i}+1}(f)=1
\end{array} \mid \mathcal{E}\left(\tau_{i}\right)\right) \leqslant P_{1}\left(X_{\tau^{\prime}-1}(f)=0\right) P_{0}\left(X_{1}(f)=1\right) \leqslant \frac{1-p}{|\Lambda|} .
$$

Combining the upper bounds for each term of the product above, we have the following upper bound for $P_{\mu}\left(\mathcal{E}\left(\tau_{m_{f}}\right)\right)$ :

$$
P_{\mu}\left(\mathcal{E}\left(\tau_{m_{f}}\right)\right) \leqslant \frac{(1-p)^{m_{f}}}{|\Lambda|^{\left|T(f) \cap\left\{1, \ldots, m_{f}-1\right\}\right|}}
$$

where

$$
\left|T(f) \cap\left\{1, \ldots, m_{f}-1\right\}\right|=\left\{\begin{array}{cl}
|J(f) \cap(T+1)| & \text { if } m_{f} \notin T(f) \\
|J(f) \cap(T+1)|-1 & \text { if } m_{f} \in T(f)
\end{array} .\right.
$$

In both cases, we have the following upper bound for (9.5):

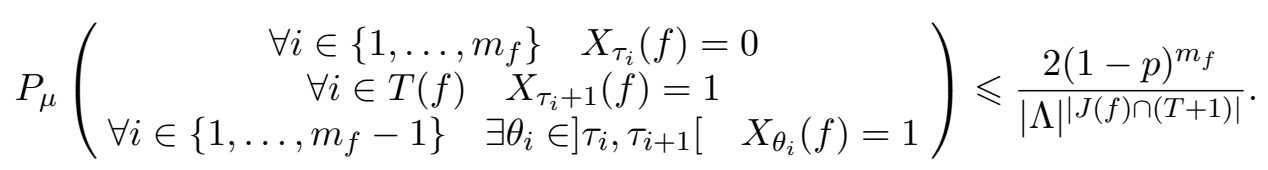

We obtain an upper bound for (9.3) by multiplying this inequality over the edges $f$ in $\operatorname{support}(\gamma)$ :

$$
P_{\mu}\left(\begin{array}{c}
\forall i \in T \quad E_{t_{i+1}}=e_{i+1} \\
\forall i \in I \backslash T \quad X_{t_{i}}\left(e_{i}\right)=0 \\
\forall p, q \in I \backslash T \text { s.t. } e_{p}=e_{q}, t_{p}>t_{q} \\
\exists r \in] t_{q}, t_{p}\left[\quad X_{r}\left(e_{p}\right)=1\right.
\end{array}\right) \leqslant \frac{2^{|S|}(1-p)^{\sum_{f \in S} m_{f}}}{|\Lambda|^{\sum_{f}|J(f) \cap(T+1)|}} \leqslant \frac{2^{|S|}(1-p)^{|I|-k}}{|\Lambda|^{k}} .
$$

Since $|I|-k \geqslant n$, and $|S| \leqslant n$, for $k$ fixed and $\left(t_{i}\right)_{i \in I}$ fixed, we have the following upper bound for $(9.1)$

$$
P\left(\begin{array}{c}
\left(e_{i}, t_{i}\right)_{i \in I} \text { is a decreasing simple impatient } \\
X \text {-closed-moving STP except on } e
\end{array}\right) \leqslant \frac{(2-2 p)^{n}}{|\Lambda|^{k}} .
$$


Finally, we use this upper bound in (9.2) and we have

$$
\begin{gathered}
P_{\mu}\left(\begin{array}{c}
\exists \gamma \text { decreasing simple impatient } \\
X \text {-closed-moving STP except on } e, \\
\gamma \text { starts from }(e, t) \text { and ends after } s, \\
\operatorname{space}(\gamma)=\left(\varepsilon_{1}, \ldots, \varepsilon_{n}\right)
\end{array}\right) \\
\quad \leqslant \sum_{1 \leqslant k \leqslant n} \sum_{A \subset\{1, \ldots, n\},|A|=k K \subset\{s, \ldots, t\},|K|=k} \frac{(2-2 p)^{n}}{|\Lambda|^{k}} \\
\leqslant \sum_{1 \leqslant k \leqslant n}\left(\begin{array}{c}
n \\
k
\end{array}\right)\left(\begin{array}{c}
\ell|\Lambda| \\
k
\end{array}\right) \frac{(2-2 p)^{n}}{|\Lambda|^{k}} \leqslant \sum_{1 \leqslant k \leqslant n}\left(\begin{array}{c}
\ell|\Lambda| \\
k
\end{array}\right) \frac{(4-4 p)^{n}}{|\Lambda|^{k}} \\
\leqslant\left(1+\frac{1}{|\Lambda|}\right)^{\ell|\Lambda|}(4-4 p)^{n} .
\end{gathered}
$$

This yields the desired result.

We use next Proposition 8.3 and Lemma 9.1 to show that the pivotal edges cannot move too fast.

Proposition 9.2. There exists $\tilde{p}<1$, such that for $p \geqslant \tilde{p}$, for $\ell \geqslant 1, t \in \mathbb{N}, s \in \mathbb{N}, s \leqslant \ell|\Lambda|$ and any edge $e$ at distance at least $\ell$ from the boundary of $\Lambda$,

$$
P_{\mu}\left(e \in \mathcal{P}_{t+s}, d\left(e, \mathcal{P}_{t} \cup \mathcal{I}_{t} \backslash\{e\}\right) \geqslant \ell\right) \leqslant \exp (-\ell)
$$

Proof: By Proposition 8.3, there exists a STP which is decreasing simple impatient and $X$-closedmoving except on $e$ which starts from the edge $e$ at time $t+s$ and ends at an edge of $\mathcal{P}_{t} \cup \mathcal{I}_{t} \backslash\{e\}$ or an edge intersecting the boundary of $\Lambda$ after the time $t$. In both cases, this STP has a length at least $\ell$. Therefore, we have the inequality

$$
P_{\mu}\left(e \in \mathcal{P}_{t+s}, d\left(e, \mathcal{P}_{t} \cup \mathcal{I}_{t} \backslash\{e\}\right) \geqslant \ell\right) \leqslant P_{\mu}\left(\begin{array}{c}
\exists \gamma \text { decreasing simple impatient } \\
X \text {-closed-moving STP except on } e \\
\gamma \text { starts from }(e, t+s) \text { and ends after } t \\
\mid \text { length }(\gamma) \mid \geqslant \ell
\end{array}\right) .
$$

Let us fix a path $\left(e_{1}, \ldots, e_{n}\right)$ with $n=\ell$ starting from $e$. By Lemma 9.1, for $\ell \geqslant 1$, we have

$$
P_{\mu}\left(\begin{array}{c}
\exists \gamma \text { decreasing simple impatient } \\
X \text {-closed-moving STP except on } e, \\
\gamma \text { starts from }(e, t+s) \text { and ends after } t \\
\operatorname{space}(\gamma)=\left(e_{1}, \ldots, e_{\ell}\right)
\end{array}\right) \leqslant\left(1+\frac{1}{|\Lambda|}\right)^{\ell|\Lambda|}(4-4 p)^{\ell} .
$$

We sum over the number of the choices for the path $\left(e_{1}, \ldots, e_{\ell}\right)$ and we obtain

$$
P_{\mu}\left(\begin{array}{c}
\exists \gamma \text { decreasing simple impatient } \\
X \text {-closed-moving STP except on } e \\
\gamma \text { starts from }(e, t+s) \text { and ends after } t \\
\mid \text { length }(\gamma) \mid \geqslant \ell
\end{array}\right) \leqslant\left(1+\frac{1}{|\Lambda|}\right)^{\ell|\Lambda|} \alpha(d)^{\ell}(4-4 p)^{\ell},
$$

where $\alpha(d)$ is the number of the $*$-neighbours of an edge in dimension $d$. There exists a $\tilde{p}<1$ such that for $p \geqslant \tilde{p}$, we have

$$
\forall \ell \geqslant 1 \quad\left(1+\frac{1}{|\Lambda|}\right)^{\ell|\Lambda|} \alpha(d)^{\ell}(4-4 p)^{\ell} \leqslant e^{-\ell} .
$$

This gives the desired upper bound. 


\section{The proof of Theorem 1.5}

We now prove Theorem 1.5 with the help of Proposition 9.2 and the observation that an edge of the interface cannot survive a time more than $O(|\Lambda| \ln |\Lambda|)$.

Proof of Theorem 1.5: Let $c$ be a constant bigger than 1. We define two sets $\mathfrak{P}_{t}^{-}$and $\mathfrak{P}_{t}^{+}$as

$$
\begin{aligned}
\mathfrak{P}_{t}^{-} & =\bigcup_{r \in[t-2 d c|\Lambda| \ln |\Lambda|, t]} \mathcal{P}_{r} \\
\mathfrak{P}_{t}^{+} & =\bigcup_{s \in[t, t+2 d c|\Lambda| \ln |\Lambda|]} \mathcal{P}_{s} .
\end{aligned}
$$

By the definition of $d_{H}^{\ell}$, we have

$$
\begin{aligned}
& P_{\mu}\left(d_{H}^{2 d c \ln |\Lambda|}\left(\mathfrak{P}_{t}^{+}, \mathfrak{P}_{t}^{-}\right) \geqslant\right.2 d c \ln |\Lambda|) \\
&=P_{\mu}\left(\begin{array}{r}
\exists s \in[0,2 d c|\Lambda| \ln |\Lambda|] \quad \exists e \in \mathcal{P}_{t+s} \\
d\left(e, \Lambda^{c} \cup \mathfrak{P}_{t}^{-}\right) \geqslant 2 d c \ln |\Lambda|
\end{array}\right) \\
& \quad+P_{\mu}\left(\begin{array}{c}
\exists s \in[0,2 d c|\Lambda| \ln |\Lambda|] \quad \exists e \in \mathcal{P}_{t-s} \\
d\left(e, \Lambda^{c} \cup \mathfrak{P}_{t}^{+}\right) \geqslant 2 d c \ln |\Lambda|
\end{array}\right) .
\end{aligned}
$$

Since the probability concerns only the process $\left(Y_{t}\right)_{t \in \mathbb{N}}$, which is reversible, we have

$$
P_{\mu}\left(\begin{array}{c}
\exists s \in[0,2 d c|\Lambda| \ln |\Lambda|] \quad \exists e \in \mathcal{P}_{t+s} \\
d\left(e, \Lambda^{c} \cup \mathfrak{P}_{t}^{-}\right) \geqslant 2 d c \ln |\Lambda|
\end{array}\right) \quad=\quad P_{\mu}\left(\begin{array}{c}
\exists s \in[0,2 d c|\Lambda| \ln |\Lambda|] \quad \exists e \in \mathcal{P}_{t-s} \\
d\left(e, \Lambda^{c} \cup \mathfrak{P}_{t}^{+}\right) \geqslant 2 d c \ln |\Lambda|
\end{array}\right) .
$$

Therefore, we can concentrate on the following probability

$$
P_{\mu}\left(\begin{array}{c}
\exists s \in[0,2 d c|\Lambda| \ln |\Lambda|] \quad \exists e \in \mathcal{P}_{s} \\
d\left(e, \Lambda^{c} \cup \mathfrak{P}_{t}^{-}\right) \geqslant 2 d c \ln |\Lambda|
\end{array}\right) .
$$

Let us fix an edge $e$ in $\Lambda$ at distance at least $2 d c \ln |\Lambda|$ from $\mathfrak{P}_{t}^{-}$and a $s \in[0,2 d c|\Lambda| \ln |\Lambda|]$. We distinguish two cases. If the set $\mathcal{P}_{t} \cup \mathcal{I}_{t}$ is at distance more than $2 d c \ln |\Lambda|$ from the edge $e$, then by Proposition 9.2, there exists a $p_{2}<1$ such that, for $p \geqslant p_{2}$ and $c \geqslant 1$, we have

$$
P_{\mu}\left(e \in \mathcal{P}_{t+s}, d\left(e, \mathcal{P}_{t} \cup \mathcal{I}_{t}\right) \geqslant 2 d c \ln |\Lambda|\right) \leqslant \exp (-2 d c \ln |\Lambda|) .
$$

If there exists an edge $f \in \mathcal{P}_{t} \cup \mathcal{I}_{t}$, which is at distance less than $2 d c \ln |\Lambda|$ from $e$, we consider the last time when $f$ was pivotal before $t$ and we define the random integer $\tau$ such that

$$
\tau=\inf \left\{r \geqslant 0: f \in \mathcal{P}_{t-r}\right\} .
$$

Since $f \notin \mathfrak{P}_{t}^{-}$, we must have $\tau \geqslant 2 d c|\Lambda| \ln |\Lambda|$. The edge $f$ is not pivotal during the time interval $[t-\tau+1, t]$ and it belongs to the interface. Moreover, it cannot be chosen to be modified during this interval since it must remain different in the two processes. Therefore, for any $r \in[t-2 d c|\Lambda| \ln |\Lambda|+$ $1, t]$, we have $E_{r} \neq f$. However, this event is unlikely because the sequence $\left(E_{t}\right)_{t \in \mathbb{N}}$ is a sequence of i.i.d. random edges chosen uniformly in $\Lambda$. More precisely, we have the following inequality:

$$
\begin{aligned}
& P_{\mu}(\tau \geqslant 2 d c|\Lambda| \ln |\Lambda|) \leqslant P\left(\forall r \in[t-2 d c|\Lambda| \ln |\Lambda|+1, t], E_{r} \neq f\right) \\
& \leqslant\left(1-\frac{1}{2 d|\Lambda|}\right)^{2 d c|\Lambda| \ln |\Lambda|} \leqslant \frac{1}{|\Lambda|^{c}}
\end{aligned}
$$


We obtain the following inequality:

$$
P_{\mu}\left(\begin{array}{c}
e \in \mathcal{P}_{t+s} \quad \exists f \in \mathcal{P}_{t} \cup \mathcal{I}_{t} \\
d(e, f)<2 d c \ln |\Lambda| \\
d\left(e, \Lambda \cup \mathfrak{P}_{t}^{-}\right) \geqslant 2 d c \ln |\Lambda|
\end{array}\right) \leqslant \frac{\lambda(d)(2 d c \ln |\Lambda|)^{d}}{|\Lambda|^{c}}
$$

where $\lambda(d)$ is a constant depending only on the dimension. We combine the two cases (10.1) and (10.2), we obtain

$$
P_{\mu}\left(e \in \mathcal{P}_{t+s}, d\left(e, \Lambda \cup \mathfrak{P}_{t}^{-}\right) \geqslant 2 d c \ln |\Lambda|\right) \leqslant \frac{\lambda(d)(2 d c \ln |\Lambda|)^{d}}{|\Lambda|^{c}}+\frac{1}{|\Lambda|^{2 d c}} .
$$

We then sum over the number of the choices for the edge $e$ and of the number $s$ from 1 to $2 d c|\Lambda| \ln |\Lambda|$. We obtain

$$
P_{\mu}\left(\begin{array}{c}
\exists s \in[t, t+2 d c|\Lambda| \ln |\Lambda|] \quad \exists e \in \mathcal{P}_{t+s} \\
d\left(e, \Lambda^{c} \cup \mathfrak{P}_{t}^{-}\right) \geqslant 2 d c \ln |\Lambda|
\end{array}\right) \leqslant \frac{\lambda(d)(2 d c \ln |\Lambda|)^{d+1}}{|\Lambda|^{c-2}}+\frac{2 d c \ln |\Lambda|}{|\Lambda|^{2 d c-2}}
$$

In other words, we have

$$
P_{\mu}\left(\mathfrak{P}_{t}^{+} \nsubseteq \mathcal{V}\left(\Lambda^{c} \cup \mathfrak{P}_{t}^{-}, 2 d c \ln |\Lambda|\right)\right) \leqslant \frac{\lambda(d)(2 d c \ln |\Lambda|)^{d+1}}{|\Lambda|^{c-2}}+\frac{2 d c \ln |\Lambda|}{|\Lambda|^{2 d c-2}}
$$

By the reversibility of the process $\left(Y_{t}\right)_{t \in \mathbb{N}}$, we also have

$$
P_{\mu}\left(\mathfrak{P}_{t}^{-} \nsubseteq \mathcal{V}\left(\Lambda^{c} \cup \mathfrak{P}_{t}^{+}, 2 d c \ln |\Lambda|\right)\right) \leqslant \frac{\lambda(d)(2 d c \ln |\Lambda|)^{d+1}}{|\Lambda|^{c-2}}+\frac{2 d c \ln |\Lambda|}{|\Lambda|^{2 d c-2}}
$$

Combining the two previous inequalities, we have

$$
P_{\mu}\left(d_{H}^{2 d c \ln |\Lambda|}\left(\mathfrak{P}_{t}^{-}, \mathfrak{P}_{t}^{+}\right) \geqslant 2 d c \ln |\Lambda|\right) \leqslant \frac{2 \lambda(d)(2 d c \ln |\Lambda|)^{d+1}}{|\Lambda|^{c-2}}+\frac{4 d c \ln |\Lambda|}{|\Lambda|^{2 d c-2}} .
$$

For $|\Lambda| \geqslant e^{2 d^{2} c}$, we have

$$
\frac{2 \lambda(d)(2 d c \ln |\Lambda|)^{d+1}}{|\Lambda|^{c-2}}+\frac{4 d c \ln |\Lambda|}{|\Lambda|^{2 d c-2}} \leqslant \frac{1}{|\Lambda|^{c-3}} .
$$

This yields the desired result.

\section{Acknowledgements}

The authors thank Jean-Baptiste Gouéré for numerous remarks and comments, which helped to improve considerably several proofs.

\section{References}

Bodineau, T. The Wulff construction in three and more dimensions. Comm. Math. Phys., 207 (1), 197-229 (1999). MR1724851.

Campanino, M., Ioffe, D., and Velenik, Y. Ornstein-Zernike theory for finite range Ising models above $T_{c}$. Probab. Theory Related Fields, 125 (3), 305-349 (2003). MR1964456.

Cerf, R. Large deviations for three dimensional supercritical percolation. Astérisque, (267), vi+177 (2000). MR1774341.

Cerf, R. and Pisztora, A. On the Wulff crystal in the Ising model. Ann. Probab., 28 (3), 947-1017 (2000). MR1797302.

Deuschel, J.-D. and Pisztora, A. Surface order large deviations for high-density percolation. Probab. Theory Related Fields, 104 (4), 467-482 (1996). MR1384041.

Dobrushin, R. The Gibbs state that describes the coexistence of phases for a three-dimensional Ising model. Teor. Verojatnost. i Primenen., 17, 619-639 (1972). MR0421546. 
Dobrushin, R. and Hryniv, O. Fluctuations of the phase boundary in the 2D Ising ferromagnet. Comm. Math. Phys., 189 (2), 395-445 (1997). MR1480026.

Dobrushin, R., Kotecký, R., and Shlosman, S. Wulff construction. A global shape from local interaction, volume 104 of Translations of Mathematical Monographs. American Mathematical Society, Providence, RI (1992). ISBN 0-8218-4563-2. MR1181197.

Gielis, G. and Grimmett, G. Rigidity of the interface in percolation and random-cluster models. $J$. Statist. Phys., 109 (1-2), 1-37 (2002). MR1927913.

Greenberg, L. and Ioffe, D. On an invariance principle for phase separation lines. Ann. Inst. H. Poincaré Probab. Statist., 41 (5), 871-885 (2005). MR2165255.

Grimmett, G. The stochastic random-cluster process and the uniqueness of random-cluster measures. Ann. Probab., 23 (4), 1461-1510 (1995). MR1379156.

Grimmett, G. Percolation, volume 321 of Grundlehren der Mathematischen Wissenschaften [Fundamental Principles of Mathematical Sciences]. Springer-Verlag, Berlin, second edition (1999). ISBN 3-540-64902-6. MR1707339.

Ioffe, D. Large deviations for the 2D Ising model: a lower bound without cluster expansions. $J$. Statist. Phys., 74 (1-2), 411-432 (1994). MR1257822.

Ioffe, D. Exact large deviation bounds up to $T_{c}$ for the Ising model in two dimensions. Probab. Theory Related Fields, 102 (3), 313-330 (1995). MR1339736.

Ioffe, D. and Schonmann, R. H. Dobrushin-Kotecký-Shlosman theorem up to the critical temperature. Comm. Math. Phys., 199 (1), 117-167 (1998). MR1660207.

Kelly, F. P. Reversibility and stochastic networks. Cambridge Mathematical Library. Cambridge University Press, Cambridge (2011). ISBN 978-1-107-40115-0. MR2828834.

Kesten, H. Aspects of first passage percolation. In École d'été de probabilités de Saint-Flour, XIV1984, volume 1180 of Lecture Notes in Math., pp. 125-264. Springer, Berlin (1986). MR876084.

Schonmann, R. H. Slow droplet-driven relaxation of stochastic Ising models in the vicinity of the phase coexistence region. Comm. Math. Phys., 161 (1), 1-49 (1994). MR1266068.

Steif, J. E. A survey of dynamical percolation. In Fractal geometry and stochastics IV, volume 61 of Progr. Probab., pp. 145-174. Birkhäuser Verlag, Basel (2009). MR2762676.

Timár, A. Boundary-connectivity via graph theory. Proc. Amer. Math. Soc., 141 (2), 475-480 (2013). MR2996951.

Zhou, W. The localisation of low-temperature interfaces in $d$ dimensional Ising model. ArXiv Mathematics e-prints (2019). arXiv: 1901.05787. 